$$
\text { NSG-xo14 }
$$

\title{
LABORATORY SIMULATION OF INFRARED ASTROPHYSICAL FEATURES
}

(NASA-CR-155939) LABORATORY SIIIULATION OF INFRARED ASTROPHYSTCAI FEATIRES Ph.D.

Thesis (Minnesota Univ.) 151 p HC A 08/MF

A 01

BY

LOUIS ALLEN ROSE

1977

REPORT No. 16

ASTROPHYSICS

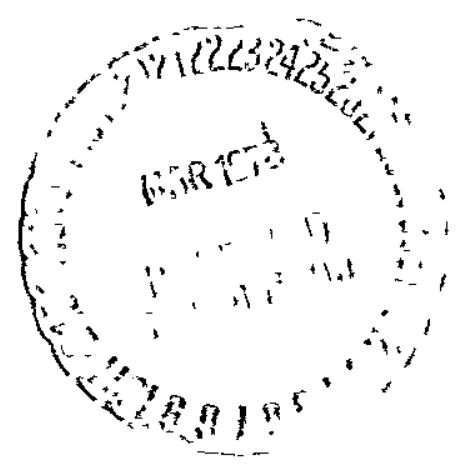

School of Physics and Astronomy

UNIVERSITY OF MINNESOTA 
LABORATORY SIMULATION OF

INFRARED ASTROPHYSICAI FEATURES

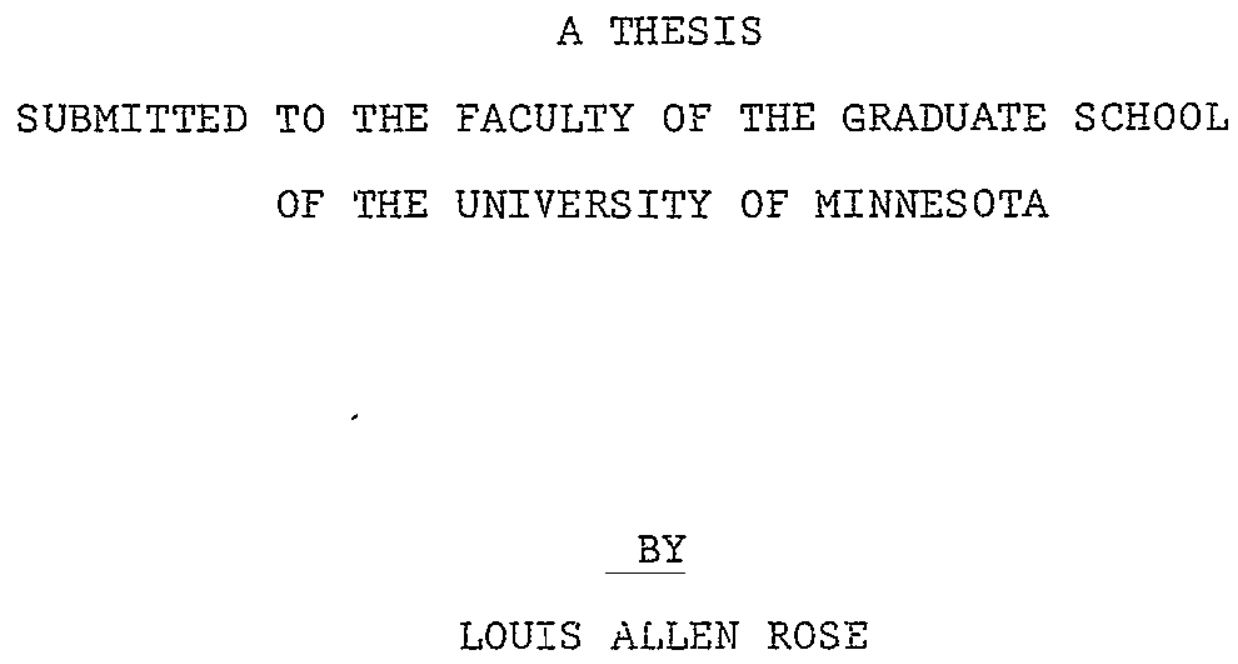

IN PARTIAL FULFILIMENT OF THE REQUIREMENTS

FOR THE DEGREE OF

DOCTOR OF PHILOSOFHY

AUGUST, 1977 


\section{ACKNOWLEDGMENTS}

Because this project was interdisciplinary between the fields of astronomy, geology and physics a large number of people have contributed ideas and assistance. First of all, I thank E. P. Ney who had the idea of a laboratory study of silicates (as it related to infrared astronomyl and who gave me a chance to work on it. He also provided valuable encouragement and assistance along the way. Discussions with Mike Merrill were extremely helpful to me in getting acquainted with the world of infrared astronomy and astrophysical solids. S. R. B. Cooke offered helpful discussions and encouragement. I thank Calvin Alexander, M. B. Duke, Rama Murthy and R. 0 . Pepin for helpful discussions and assistance in securing Iunar and meteoritic materials. I thank J. B. Adams for helpful discussions and for sponsoring the portion of this study related to lunar samples. Paul Weiblen and Jim Stout offered advice and assistance in securing terrestrial samples. I thank M. R. Coscio, Burt Bulato, Norman Shultz, John Broadhurst and George Idzorik for help in sample preparation. I thank Jim Stoddart for engineering assistance. 
Finally, I thank my wlfe Emmanelle, my chlldren Cindy Beth, Ieslyn and Andrew, my parents Lonnie and Vivian Rose and my wife's parents, Brodie and Bobbie Owens for their love, help, patience and understanding. 
Louis Allen Rose

\author{
IABORATORY SIMULATION OF \\ INFRARED ASTROPHYSICAL FEATURES
}

\begin{abstract}
Laboratory infrared emission and absorption spectra have been taken of terrestrial silicates, meteorites and Iunar soils in the form of micrometer and sub-micrometer grains. The emission spectra were taken in a way that imitates telescope observations. The purpose was to see which materials best simulate the $10 \mu \mathrm{m}$ astrophysical feature.

The emission spectra of forsterite, fayalite and Allende give a good fit to the $10 \mu \mathrm{m}$ broadband emission feature of Comets Bennett and Kohoutek. Quartz can be ruled out as a major component of cometary grains since its silicate feature occurs near $9 \mu \mathrm{m}$ and does not matah the astrophysical emission feature. A study of the effect of grain size on the presence of the $10 \mu \mathrm{m}$ emission feature of forsterite shows that for particles larger than $37 \mu \mathrm{m}$ no feature is seen, but the feature is seen for particles smaller than this.
\end{abstract}


- Iovis Allen Rose

Intermediate resolution emission spectroscopy was used to study a group of 9 terrestrial sillcates, 1 synthetic silicate, 6 meteorites and 2 lunar soils; comparisons were made with the intermediate resolution spectra of Comet Kohoutek. Infrared absorption spectra were taken of selected materials from this group.

This study has found three mixtures of silicates which would yield spectra that match the spectrum of

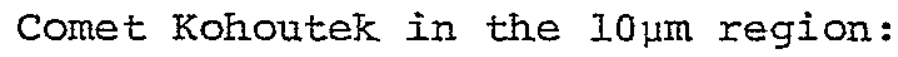

1) A hydrous layer lattice silicate in combination with a high temperature condensate such as olivine or anorthite to broaden the feature; 2). an amorphous magnesium silicate in combination with a high temperature condensate and 31 glassy olivine and glassy anorthite in approximately equal proportions. Mixture 1 would be fairly close in composition to a C2 meteorite. Mixtures 1 and 2 would both form partly in a low temperature environment. Mixture 3 is made of high temperature condensates which condense and cool in such a way so as to produce amorphous grains. 


\section{CONTENTS}

INTRODUCTION $\ldots \ldots \ldots \ldots \ldots \ldots \ldots \ldots \ldots \ldots \ldots$

CHAPTER 1 DUST AND THE 10 UM ASTROPHYSICAL FEATURE. 3

I The $10 \mu \mathrm{m}$ Feature in Emission ......... 3

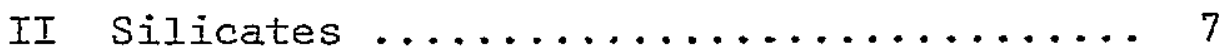

III Dust Formation in M Stars .......... 12

IV Dust Condensation Theories ......... I3

$V$ Dust in the Galaxy ............... 17

VI The I0 1 m Feature in Absorption ....... 25

VII Intenstellar Extinction ............ 29

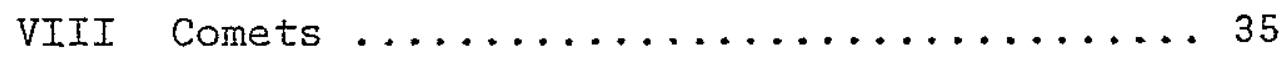

IX Laboratory Measurements of Silicates ... 45

CHAPTER 2 LABORATORY INFRARED STUDIES OF SILICATES 53

I Sample Preparation .............. 53

II ExperimentaI ............... 56

III Materials ..................... 59

IV Laboratory Broadband Spectra ........62 62

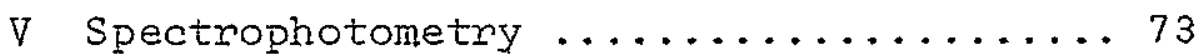

VI Summany and Conclusions .............102 APPENDIX A HANDLING SPECTROMETER DATA . . . . . . . I06 APPENDIX B A THIN FILM EXPERIMENT ............... 26 APPENDIX C NORMATIVE MINERAI, COMPOSITIONS OF CERTAIN SAMPLES ....................

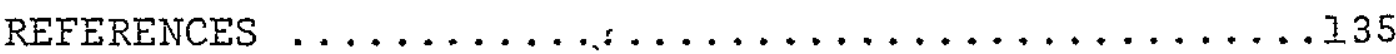




\section{ILIUSTRATIONS}

Fig. I Broadband Spectrum of $\mu$ cep.......... 5

Fig. 2 Intermediate Resolution Spectrum of $\mu$ Cep....................... 6

Fig. 3 Emission Spectrum of Quartz.......... 9

Fig. 4 Absorption Spectrum of Quartz......... 9

Fig. 5 Spectral Observations of the BN-KL Complex..................... 26

Fig. 6 Broadband Spectrum of Comet Kohoutek..... 38

Fig. 7 Sedimentation Time in Acetone (Stokes' Law) ................ 55

Fig. 8 Experimental Arrangements........... 58

Fig. 9 Laboratory Broadband Emission Spectrum of Fayalite.................... 64

Fig. 10 Summary of Laboratory Broadband Emission Spectra..................... 69

Fig. II Laboratory Broadband Emission Spectra of Allende.................. 72

Fig. 12 Laboratory Spectra of Dunite Samples with Different Grain Sizes............

Fig. 13 Laboratory Intermediate Resolution. Emission Spectrum of Dunite............

Fig. I4 Spectrum of Comet Kohoutek: Laboratory Emission Spectra of Olivines, Amorphous Silicates and an Enstatite Chondrite....

Fig. 15 Laboratory Emission Spectra of Carbonaceous Chondrites and a

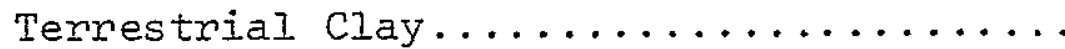

Fig. 16 Laboratory Emission Spectra of Plagioclase Feldspars, a Lunar Soil

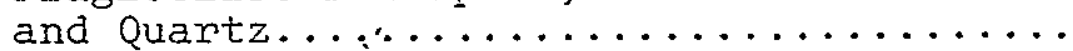




\section{ILLUSTRATIONS (con't)}

Fig. 17 Laboratory Emission Spectra of a Lunar

Mare Soil, a Basaltic Achondrite, a

Terrestrial Diabase and a Terrestrial

Basalt..................... 90

Fig. 18 Laboratory Absorption Spectra of Slag,

Amorphous Silicates and Montmorillonite. 93

Fig. 19 Laboratory Emission Spectra of Slag,

Amorphous Silicates and Montmorillonite. 94

Fig. 20 Laboratory Absorption Spectra of

Anorthite, Juvinas and Lunar Soils..... 96

Fig. 21 Laboratory Emission Spectra of

Anorthite, Juvinas and Lunar Soils..... 97

Fig. 22 Laboratory Absorption Spectra of

Melțed and Unmelted Silicates........ 100

Fig. 23 Laboratory Emission Spectra of

Melted Glassy Silicates.............. 101

Fig. A-I Chart Recorder Curve of Laboratory

Blackbody in the $3=5.5 \mu \mathrm{m}$ Range........ 107

Fig. A-2 Chart Recorden Curve of Laboratory

BIackbody in the 7-14um Range........ 108

Fig. A-3 Chart Recorden Curve of Polystyrene in

the $3-5.5 \mu \mathrm{m}$ Range............... 110

Fig. A-4 Chart Recorder Curve of Polystyrene in

the 7 -I $4 \mu \mathrm{m}$ Range.................... 111

Fig. A-5 Wavelength-Angle Plot of Spectrometer

Filter wheel in $3-5.5 \mu \mathrm{m}$ Range........ 113

Fig. A-6 Wavelength-Angle Plot of Spectrometer

Filter Wheel in 8 -I $4 \mu m$ Range......... 114

Fig. A-7 Chart Recorder Curve of Melted

Anorthite in the $3-5.5 \mu \mathrm{m}$ Range........ 115

Fig. A-8 Chart Recorder Curve of Melted

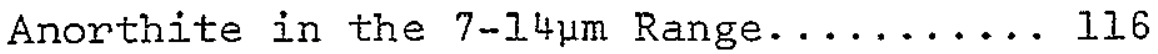




\section{ILIUSTRATIONS ( $\left.\operatorname{con}^{\dagger} t\right)$}

Fig. A-9 Chart Recorder Curve of Blank Mirror in the $3-5.5 \mu \mathrm{m}$ Range.............118

Fig. A-10 Chant Recorder Curve of Blank Mirror in the $7-14 \mu \mathrm{m}$ Range.................119

Fig. A-11 Laboratory Emission Spectra of Melted Anorthite Sample............... 125

Fig. B-1 Sio Thin Film Transmission........... I28

Fig. B-2 Plot of SiO Thin Film Transmission versus Film Thickness............ 130 


\section{TABLES}

Table 1 Description of Samples Used in This study...................... 60

Table 2 Broadband Fayalite Data............ 65

Table A-I Detector Signal Voltage For Melted Anorthite Run............. 121

Table A-2 Calculated Magnitudes and Grain Enission of Melted Anorthite......... I23

Table B-l SiO Thin Film Depositions............ I29 


\section{INTRODUCTION}

This thesis reports the results of a study undertaken to find a material that simulates in laboratory experiments the loum feature which is seen in emission or in absorption in the energy spectra of various astrophysical objects. Dust or particles in fine grain form are believed to be responsible for this spectral feature and therefore the feacure is a clue nature has given regarding the chemical composition, structure, and size of astrophysical dust particles.

Dust is an important component of our galaxy. It is part of the material from which stars are born; it is thought to be formed in a cooling nebula of solar composition; and it is manufactured and dispersed into interstellar space by certain stars in their old age. Dust is sometimes formed in one of nature's most spectacular events, a nova, when a shell of material is expelled from a stellar object. Therefone it is important to gain as much information as possible from a study of the loum spectral feature. It is a part of the astrophysical ledger to be read and understood.

The method of approach used in this study was to obtain laboratory spectra of materials under condivions that simulate certain aspects of the astrophysical environment and to compare these spectra with infrared spectra of astrophysical objects. Through 
these comparisons certain conclusions have been drawn regarding dust grains.

Chapter 1 gives some of the results of extensive infrared observations of dusty objects in our galaxy and in particular of those that show the 10um feature. The physics of emission and absorption by silicare materials is discussed as well as theories of grain formation in the atmosphere of stellar objects. The efforts of other research workers to establish some of the properties of astrophysical grains are mentioned.

Chapter 2 contains the details of sample preparation, experimental methods, and the laboratory emission and absorption spectra of the materials which have been studied. The comparisons between laboratory spectra and astrophysical spectra form the basis of the conclusions at the end of this chapter. 
CHAPTER I

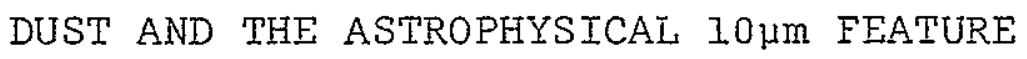

This chapter reviews some of the research work in infrared astrophysics which led up to this study. This work includes observational programs that obtained and interpreted infrared spectra of stars and other astrophysical objects, theoretical studies of the infrared emission and absorption of dust particles, and laboratory studies of materials that are thought to be possible components of astrophysical solids.

\section{THE 10 HM FEATURE IN EMISSTON}

Woolf and Ney (1969) identified an emission Feature near 10um in the spectra of two M giant Mira variable stars and two $M$ supergiant stars. These four stars had been studied earlier by Gillett, Low and Stein (1969) using an intermediate resolution infrared spectrometer. They reported an emission peak near $10 \mu \mathrm{m}$ in the spectrum of the star X Cyg; but due to uncertainties about the relative calibration of the

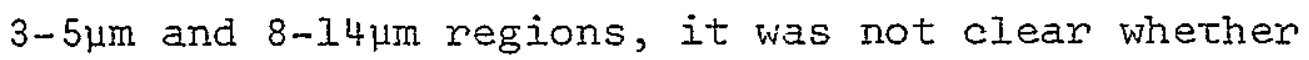
the other three stans in the group showed, absorption in a band from 7.5 to $9.5 \mu \mathrm{m}$ or emission in the 9.5 $14 \mu \mathrm{m}$ range. Woolf and Ney attributed the emission feature to circumstellar matter in the form of fine 
dust particies.

Figure $I$ shows a broadband spectrum of the $M$ supergiant $\mu$ Cep (Maas et al. 1970). The wavelengths of observation in the infrared were chosen to correspond to atmospheric windows on gaps in the molecular absorption bands. $F_{\lambda}$ is the energy flux from the stan (typical units are watts $/ \mathrm{cm}^{2} \mu \mathrm{m}$ ) and $\lambda$ is the wavelength of observation. The spectrum of $\mu$ cep has the general appearance of a thermal continuum with an emission peak superimposed at loum. The thermal portion of the spectrum is due to emission from material in the photosphere of the star at a temperature characteristic of the star's spectral type. Figure 2 is another spectrum of $\mu$ Cep using intermediate resolution spectrophotometry for which $\Delta \lambda / \lambda=.01$ (Russell et al. 1975). Here again the spectrum is that of a stellar continuum shining through an optically thin circumstellar shell which contributes the emission feature in the $10 \mu \mathrm{m}$ range. The greater resolution in this figure shows a smooth feature with little or no structure which peaks near 9.7 or $9.8 \mu \mathrm{m}$. Extensive observations have been reported of highly evolved late type oxygen-rich stars in the $8-14 \mu \mathrm{m}$ range using intermediate resolution spectroscopy (Forrest et al., 1975; Merrill and 


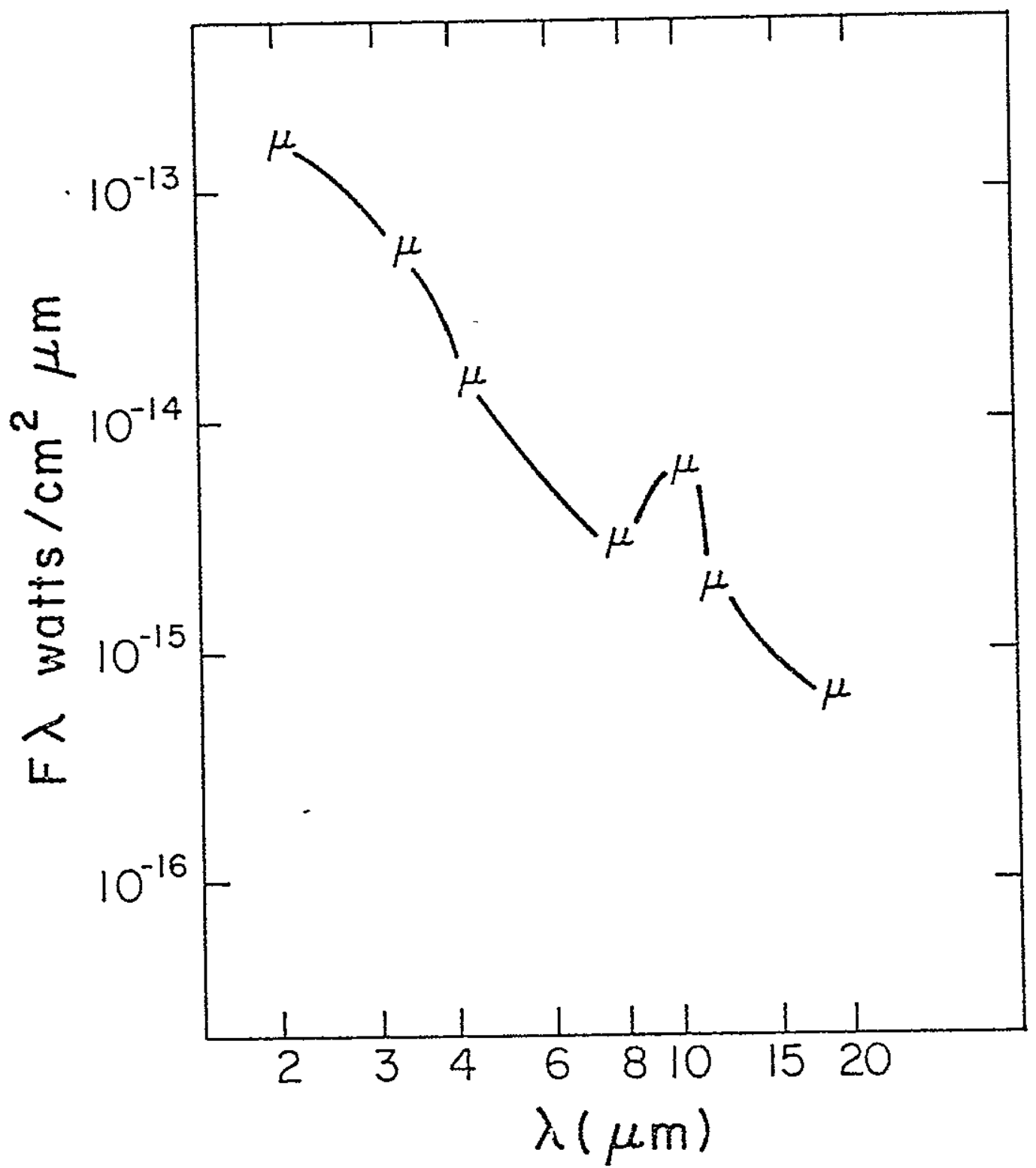

Fig. 1. Broadband photometry of $\mu$ Cep. From Maas, R.W., Ney, E.P. and Woolf, N.J. 1970, Ap.J. (Letters) 160, L101. 


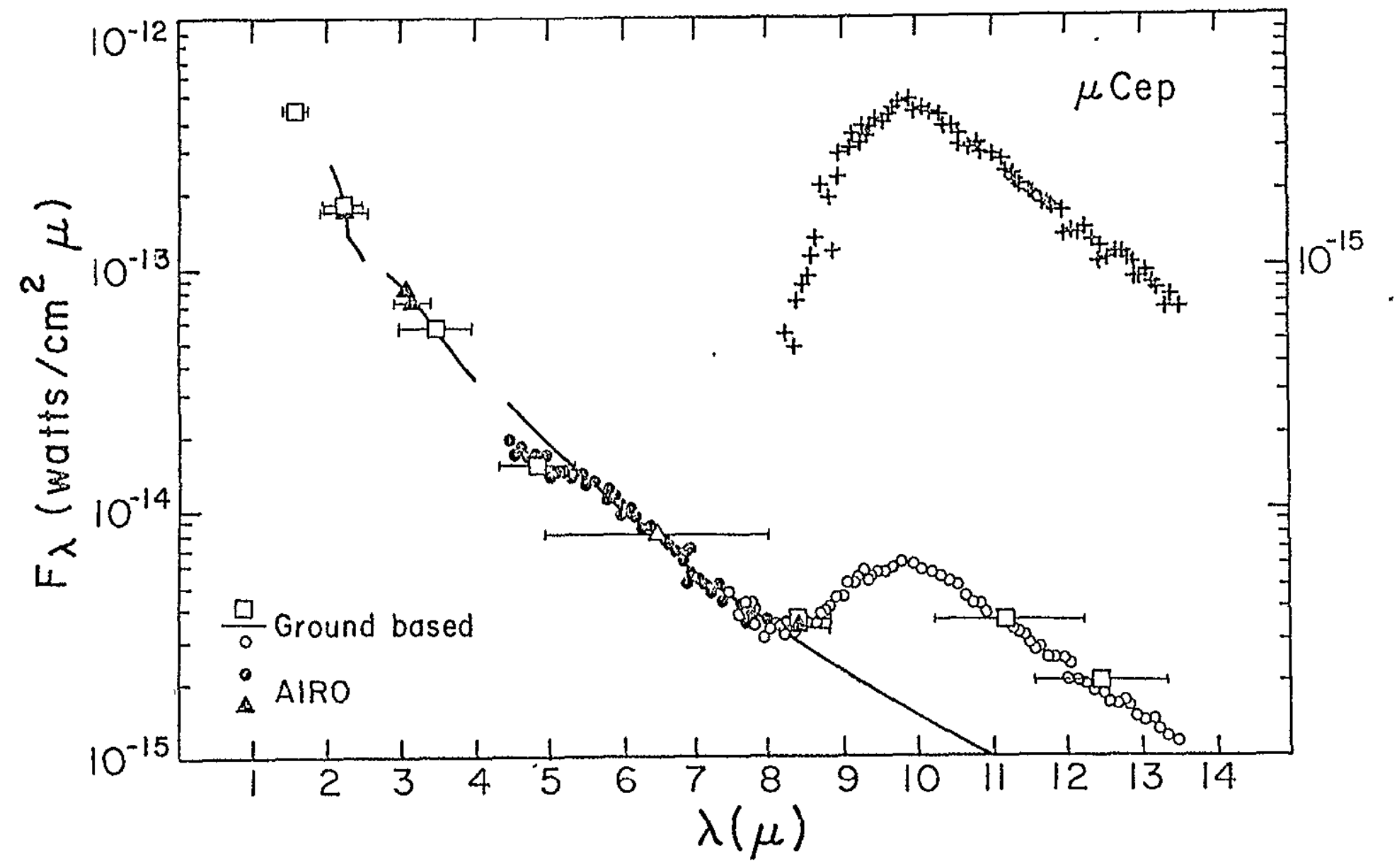

Fig. 2. The spectrum of $\mu$ cep. The heavy line is a fit of a $4200^{\circ} \mathrm{K}$ blackbody to the or

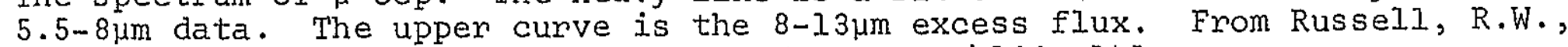
Soifer, B.T. and Fomest, W.J. 1975, Ap.J. (Letters) 198, L41. 
Stein 1976a, 1976b). In many cases the spectra resemble the one shown in Figure 2 for $\mu \mathrm{Cep}$ and have a smoothly varying feature that peaks near $9.7 \mu \mathrm{m}$. Also in the case of broadband observations the spectra of $M$ stars showing the optically thin silicate feature resemble the spectra shown in Figure l. High resolution spectra $(\Delta \lambda / \lambda=.004)$ have been taken of the star o Cet (spectral type M6-M9) by Gammon, Gausted and Treffers (1972) using a rapid scanning Michelson interferometer. The resulting spectrum was smooth possessing no fine structure.

\section{SILICATES}

What kind of materials in the circumstellan shells of $M$ stars could give rise to the $10 \mu \mathrm{m}$ emission feature? Woolf and Ney (I969) proposed silicates, the minerals containing silicon, oxygen and one or more metals, because they have an absorption mode near loum due to Si-O stretching motions (Hunt and Salisbury 1974) and because solid silicate materials are expected to condense in the cool atmospheres of late type oxygen-rich stars. Hunt and Logan (1972) have obtained laboratory emission spectra of silicates. Some of the samples they used were optically thin so that emission from individual particles escaped without significant interaction with 
other particles in the sample. In this case the emission maxima correspond in wavelength to the so called restrahlen features due to the silicon-oxygen stretching fundamental vibrational modes. (See Figure 3.) From Kirchoff's Law it is known that an object shows high emission at those wavelengths for which it also has high absorption. Figure 4 shows the absorption spectrum of a cloud of quartz particles to emphasize the agreement in wavelength of emission and absorption of optically thin samples of small silicate grains (Hunt et al. 1973).

Silicates have a second absorption band near 20um due to a Si-O-Si bending mode (Hunt and Salisbury 1974); an emission feature near $20 \mu \mathrm{m}$ in the spectrum of a Ori, one of the first stars in which the loum feature was seen, was reported by Low and Swamy (1970) giving added strength to the silicate hypothesis.

When Woolf and Ney (1969) proposed silicate dust as the source of the $10 \mu \mathrm{m}$ emission feature in $M$ stars they compared the spectrum of $\mu$ Cep, taken from the paper by Gillett, Low, and Stein (1968), with the theoretical enissivity of a silicate mixture which had been studied by Gausted (1963). Guasted published a table in the $8+15 \mu \mathrm{m}$ wavelength range of absorption properties of a mixture of mineral grains 


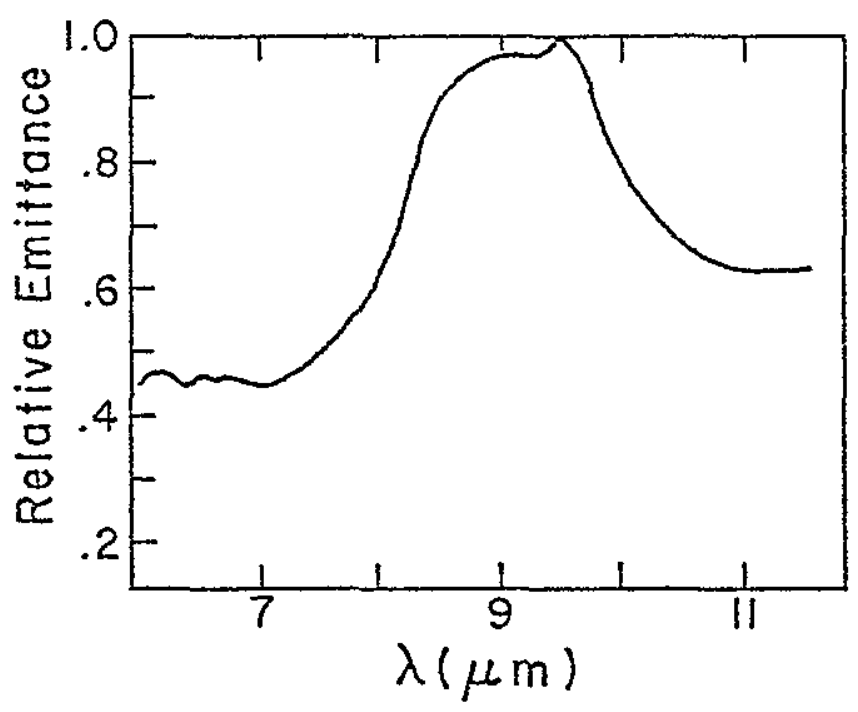

Fig. 3. Relative emittance of quartz in the $0-5 \mu \mathrm{m}$ size range. From fiunt, G.R. and Logan, L.M. 1972, Applied Optics 11, 142.

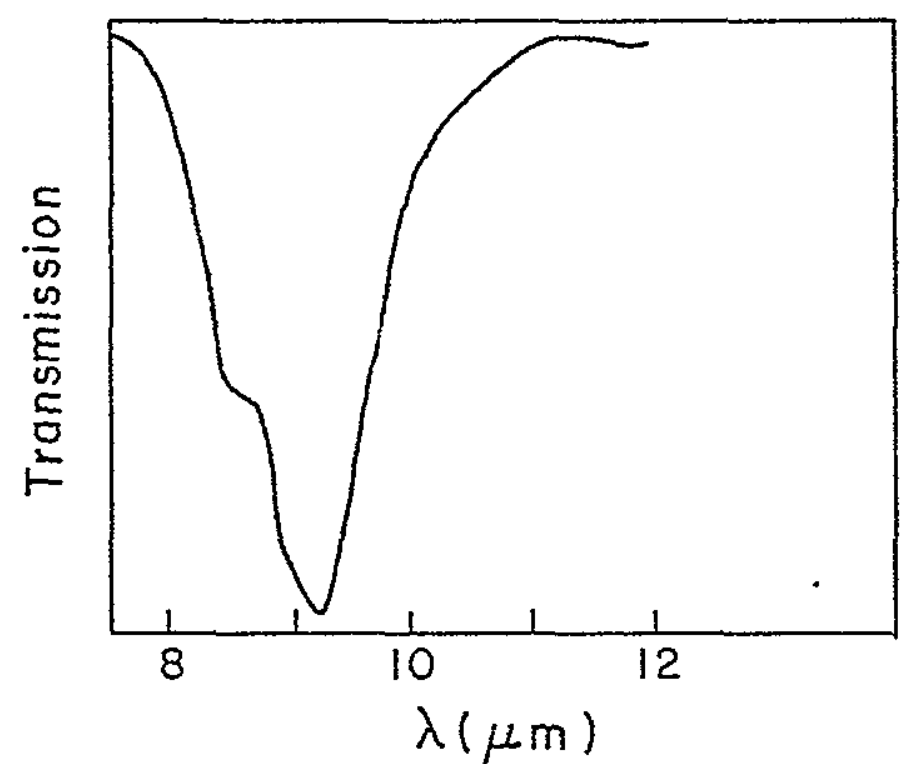

Fig. 4. Transmission spectrum of a cloud of less than $5 \mu \mathrm{m}$ particles of quartz. From Hunt, G.R., Logan, L.M. aná Salisbury, J.W. I973, Icarus 18, 459 . 
composed of enstatite $\left(\mathrm{MgSiO}_{3}\right)$, hemarite $\left(\mathrm{Fe}_{2} \mathrm{O}_{3}\right)$ and quartz $\left(\mathrm{SiO}_{2}\right)$. Enstarite constituted $80 \%$ by volune of the mixture. In detail the fit was far from perfect because the emissivity of the silicate mixture showed considerable structure which was not present in the spectrum of $\mu \mathrm{Cep}$; however, the wavelength dependence of the relative emissivity of the mixture agreed in a rough approximation with the emission spectrum of $\mu$ Cep. The percentage $\mathrm{SiO}_{2}$ by mass in the Gausted mixture is in the 60-70\% range. This is not too far from the percentage of $\mathrm{SiO}_{2}$ in dust grains around cool M stars as estimated by Woolf (1973b) who plotted frequency of maximum absorption versus percentage $\mathrm{SiO}_{2}$ for a group of siliceous rocks studied by Lyon (1964). From this graph the percentage $\mathrm{SiO}_{2}$ corresponding to a $9.7 \mu \mathrm{m}$ emission peak is $\sim 72$. Launer (1952) found that the tektosilicates such as quartz, $\mathrm{SiO}_{2}$, which are characterized by a high degree of polymerization of the $\mathrm{SiO}_{4}$ tetrahedra, the basic silicate building block, have absorption on emission peaks at wavelengths usuaily shorter than loum and that the nesosilicates such as olivine, $(\mathrm{Mg}, \mathrm{Fe}){ }_{2} \mathrm{SiO}_{4}$, which are composed of isolated tetrahedra held together by metallic ions, have peaks at wavelengths usually longen than loum. Intermediate between these 
two, both in degree of polymerization and in the wavelength peak of absorption, are the inosilicates. Gammon, Gausted and Treffers (1972) concluded that quartz emission peaks at too short a wavelength to match the astrophysical absorption feature.

- The peak opacity of terrestrial silicates near Ioum is quite high, ranging from $\sim 4000 \mathrm{~cm}^{2} / \mathrm{gm}$ for powdered olivine to $8000 \mathrm{~cm}^{2} / \mathrm{gm}$ for powdered quartz (Day et al. 1974). If astrophysical grains have opacities which are smaller than this, due to a lack of structure, then perhaps a value of $\sim 2000 \mathrm{~cm}^{2} / \mathrm{gm}$ is more appropriate. The density and opacity of olivine can be used to estimate the size of a grain which has an optical depth, $\tau$, of $I$ at the peak absorption wavelength near $10 \mu \mathrm{m}$, using the relation

$$
\tau=(X) \cdot(p) \cdot d
$$

where $x$ is the mass absorption coefficient in $\mathrm{cm}^{2} / \mathrm{gm}$, $\rho$ is the density in $\mathrm{gm} / \mathrm{cm}^{3}$ and $d$ is the grain diameter in $\mathrm{cm}$. Since the density is approximately 3.3 ,

$$
d=\frac{1}{\left(4 \times 10^{3}\right)(3.3)} \sim 1 \mu \mathrm{m} .
$$

This then is a useful estimate of the size of an astrophysical dust grain which would show a variation of emissivity with wavelength. 


\section{DUST FORMATION IN M STARS}

The pressures and temperatures appropriate for the condensation of dust grains are thought to exist in the extended and relatively cool atmospheres of $M$ stars for which the atmospheric scale heights are comparable to the size of the star. Radiation pressure on the grains leads to mass loss as both grains and gas are driven away, the grains being momentum coupled to the gas. Gehrz and Woolf (1971) have concluded that the rate of mass loss from $M$ giant and supergiant stars is adequate to explain the abundance of silicate dust in interstellar space. The $M$ giants are the main manufacturers of the product since they are more abundant than supergiants. Dust grains absorb radiant energy from the central star and re-radiate in the infrared. If the circumstellar shell of material is optically thick the visual radiation is greatly extinguished and most of the star's radiated energy appears in the infrared. However, if the star's dust shell is optically thin, the vïsual continuum radiation characteristic of the star's spectral class shines through and silicates, if present in the shell, will contribute infrared excesses at 10 and $20 \mu \mathrm{m}$ : 


\section{DUST CONDENSATION THEORIES}

Gilman (1969) has described the species of grains which are expected to condense at temperatures and pressures relevant to the upper atmospheres of red giant stars using the abundance ratio of oxygen to carbon as a parameter. His equilibrium thermochemical calculations predict silicate condensations in oxygen rich stellar atmospheres in the following condensation sequence: aluminum silicates, calcium magnesium silicates, magnesium silicates and metallic iron. Three possible refractory materials mentioned by Gilman are $\mathrm{Al}_{2} \mathrm{SiO}_{5}$ which would form early in the condensation sequence because of its high condensation temperature, ${ }_{C}$, diopside $\left(\operatorname{CaMgSi}_{2} \mathrm{O}_{6}\right)$, and forsterite $\left(\mathrm{Mg}_{2} \mathrm{SiO}_{4}\right)$. Magnesium silicates similar to the one just mentioned are important because of the relatively high abundance of magnesium.

For the case of carbon rich stars, the two condensates which appear first according to Gilman are graphite and silicon carbide (SiC). The possibility of graphite condensations in stellar atmosphieres has been studied by several workers and was mentioned by O'Keefe (1939) as the cause of the changes in brightness of the carbon star. $R$ Corona Borealis. Donn et al. (1968) concluded that graphite grains can form 
in carbon stars and that these stars can produce the required amount of graphite grains to account for the interstellar extinction.

Grossman and Larimer (1974) calculated the condensation sequence of elements and compounds in a cooling nebula of solar proportions using equilibrium thermo-dynamics. There is general agreement between their results and the results of Gilman. Grossman and Larimer compare their calculated compositions to those of meteorites and give good descriptions of chondrite meteorites. The materials which precipitate above $1250^{\circ} \mathrm{K}$ are classified as refractories. Calciumaluminum silicates appear when the temperature has dropped to approximately $1600^{\circ} \mathrm{K}$; magnesium silicates appear at approximately $1350^{\circ} \mathrm{K}$. At around $700^{\circ} \mathrm{K}$ metallic iron oxidizes, combining to some extent with magnesium silicates to produce $(\mathrm{Mg}, \mathrm{Fe}) \mathrm{SiO}_{3} \cdot \mathrm{Below}^{\circ}$ $350^{\circ} \mathrm{K}$ magnesium silicates react with water forming hydrated silicates. Hydrated lattice layer silicates are the major mineral form in the Type I carbonaceous chondrites (C1). This class of meteorites is thought to be the most primitive material in the solar system, possessing the nonvolatile elements in approximately solar proportions and having been little affected by post formational physical on chemical processes 
(Zaikowski et al. 1975a, Wood 1968).' Cameron (1973) noted the apparent primordial nature of $\mathrm{Cl}$ meteorites in an article entitled "Interstellar Grains in Museums" and advocated a study of this class of meteorites stating that possibly they are collections of interstellar grains which have suffered only mild transformations. The Type 2 carbonaceous chondrites (C2) are composed of a matrix material similar to that in Cl meteorites but containing in addition a coarse grained fraction called chondrules. These chondrules, ranging from $.3 \mathrm{~mm}$ to $1 \mathrm{~cm}$ in diameter seem to be the solidification products of rapidly quenched molten droplets. The chondrules are composed of the high temperature condensates--olivine, pyroxene and feldspar. The Type 3 carbonaceous chondrites (C3) contain only small amounts of the hydrated layer lattice silicates; thein composition is dominated by olivines and pyroxenes. The $\mathrm{C} 3$ meteorites contain inclusions rich in the very high temperature condensates such as the exotic minerals perovskite $\left(\mathrm{CaTiO}_{3}\right)$, gehlenite $\left(\mathrm{Ca}_{2} \mathrm{Al}_{2} \mathrm{SiO}_{7}\right)$, and spineI $\left(\mathrm{MgAl}_{2} \mathrm{O}_{4}\right)$. These Ca-Al rich inclusions represent the highest temperature condensates from a gas of solar composition and are possibly the most primicive solids in the solar system. "The striking resemblance between the 
observed mineralogy and mineral compositions and the predicted high-temperature condensate phase assemblage is truly remarkable" wrote Grossman and Lariner (1974) when discussing these inclusions in $C 3$ meteorites. Anderson (1973) has noted the agreement in composition between the Ca-Al rich inclusions, which are low in $F e, M g$, and volatiles, and the Iunar surface.

Some research workers feel that grain condensations occur under non-equilibrium conditions and are Frimarily irreversible kinetic processes (Donn 1976). Arrhenius and Alfven (1971) proposed a model in which primordial gas is gradually added to the circumstellar region in which the medium is partially ionized. In this model the condensed grains are much cooler than the ions, electrons and atoms of the gas, the mechanism for cooling being thermal radiation into a medium which is optically thin at infrared wavelengths. If this is the case it does not appear that a particular condensation sequence must be followed and most minerals found in $\mathrm{Cl}$ meteorites could have formed in their present structural forn. For example, phyllosilicates are likely to be direct vapor condensates rather than alterations of anhydrous phases.

It should be pointed out that dust formation in 
a cooling nebula as described by Grossman and Larimer is related to the birch of a star. The proto sun occupies the central part of the nebula whlle farther out in portions of the gas cloud which are cool enough dust grains are condensing. In contrast to this, dust formation in an $M$ star occurs late in the star's life when it is in a fairly advanced stage of evolution. It is possible that the dust forming in $M$ stars is more closely related to the refractory materials in a condensation sequence outlined by Grossman and Lariner and will not contain low temperature materials such as hydrated silicates and ice. Since comets and meteorites are composed of solar system condensates, the full run of materials from refractories to low temperature hydrated silicates are expected and in fact are found in the carbonaceous chondrites.

\section{DUST IN THE GAIAXY}

Since dust is associated with many types of objects in the galaxy, a brief mention will be made here of various circumstances in which dust is observed to exist with particular emphasis on objects that display the loum feature in their infrared spectra. 
Ic variable stars are a class of late type supergiants exhibiting long period irregular variability. A group of this class of stars was studied by Gehsz, Ney and Strecker (1970); they found most had a loum feature of the type expected from an optically thin circumstellar shell of silicate particles, i.e., the infrared spectrum was that corresponding to the star's spectral class with the Ioun feature superimposed. Two of the objects had negligible excesses and they also had B stan companions, suggesting that the presence of a hot companion star inhibits formation of dust shells. These observations by Gehrz et al. also suggest that high luminosity in stars of late spectral type leads to a very pronounced loum feature.

A group of $M$ supergiants in Carina has been observed by Humphreys, Strecker and Ney (1972). For three type $M 4$ supergiants in this group having optically thin dust shells, it was found that the more luminous the star, the larger the loum excess. Three of the oDjects, VY CMa, VX Sgr and HD 97671, observed by Humphreys et al. (1972) displayed a silicate feature superimposed on a non-PIanckian infrared continuum. The "dust thermosphere" described by these authors consisted of an extensive cloud of dust 
at various distances from the star, which must necessarily be at different temperatures. Therefore, the infrared radiation from these objects showed excesses from 1 to $20 \mu \mathrm{m}$ and could be interpreted as a superposition of a lange number of blackbody curves. The I0um feature has also been seen in the spectra of $G$ supergiants (Humphreys, Strecker and Ney 1971). For this class of stars the region above its $5000^{\circ} \mathrm{K}$ photosphere is probably too hot for grain formation and it has been hypothesized that dust grains formed during an earlier $M$ star stage during which the star's atmosphere was cooler.

Carbon stars are red giants that have spectra showing strong bands of the carbon compounds and extremely weak metallic oxide bands. They are rare. only approximately $10^{5}$ are estimated to exist in the galaxy and eighty per cent of these are $N$ stars with effective temperatures ranging from $3000^{\circ} \mathrm{K}$ downward to $1500^{\circ} \mathrm{K}$. R Coronae Borealis is a carbon star that has been observed in the infrared. It has an infrared excess believed to be coming from a circumstellar shell of graphite and silicon carbide with a temperature of $\sim 940^{\circ} \mathrm{K}$, and its rather random change of brightness in the visual has been interpreted as being due to the chance motion of dust clouds into 
the star's Iine of sight (O'Keefe 1939; Stein et al. 1969a). A large number of observations of carbon stars have been made by Forrest, Gillett and Stein (1975) and by Merrill and Stein (1976a, 1976b). In some cases the infrared excess radiation is that of a thermal continuum produced perhaps by graphite grains and a feature in the 10 to $12.5 \mu \mathrm{m}$ region which has been attributed to silicon carbide (Treffers and Cohen 1974). The carbon Mira variables V Cyg and $T$ Dra fit in this category.

The I0Hm silicate feature has been observed in the spectra of young-hot $O$ and $B$ stars in the Orion Trapezium and in the infrared spectra of regions between the stars of the Trapezium. Stein and Gillett (1969b) showed that the broadband I0um feature of the region between the stars is very similar to that of $\mu \mathrm{Cep}$, an $M$ supergiant. The dusty region in orion is very large in extent and it is thought that the dust around the young orion stars may be a part of the cloud from which they were formed. The material is heated by the stars and blown out by radiation, the mass outflow process being halted when the kinetic pressure of the surrounding HII region becomes equal to the radiation pressure of the central star on the grains; the temperature 
and optical depth of the dust, shells surrounding the $O$ and $B$ stars in Orion have been estimated as being approximately $200^{\circ} \mathrm{K}$ and $10^{-4}$ respectively (Ney et al. 1973).

Some stars are embedded in clouds so thick that stèllar spectral features can hardly be seen. NML Cyg is such an object. It exhibits a thermal continuum corresponding to a temperature of $1500^{\circ} \mathrm{K}$. This thermal radiation is coming presumably from circumstellar grains in optically thick clouds so that no silicate signature can be seen. The grains are absorbing the stellar radiation and radiating in the infrared. It is thought that NML Cyg is a late-type supergiant; Johnson (1966) has assigned it the spectral classification M6 Ia. There are approximately 50 known cygnids; i.e., the class of objects that radiate as cool blackbodies for which a circumstellar shell obscures the central star (Ney 1977a).

Some stars with thick circumstellar shells have an associated nebulosity. VY Canis Majoris is a. bright red star embedded in a nebulosity which has clumps or condensations. It is one of the brightest objects in the sky at $10 \mu \mathrm{m}$ and, as has been mentioned, its non-Planckian infrared spectrum shows a weak feature at $10 \mu \mathrm{m}$. The infrared radiation is attributed 
to a disk-shaped nebula (containing silicate dust) immediately surrounding the star. In an extended region around the star high levels of polarization in the visible are seen with the direction of the $H$ vector always pointing along radial lines to the central star (Herbig 1972). The material which causes the polarization is thought to be compositionally the same as the dust closer in which is the source of the high infrared luminosity in the 1 to $20 \mu \mathrm{m}$ range. However this material is farther out, cooler and much thinner optically than the central disk. The polarization is caused by scattering of the visible photons on dust grains which are small compared to wavelengths of visible light.

The egg rebula is an object which displays some of the features seen in both NML Cyg and VY Canis Majoris but with a personality all its own (Ney 1975). It was discovered as an object very bright at $10 \mu \mathrm{m}$ but not listed in the Neugebauen-Leighton $2.2 \mu \mathrm{m}$ catalogue of infrared stars. Subsequent observations showed a visual double nebulosity with an infrared source situated between the visual components. The spectrum of the infrared source corresponds to a $150^{\circ} \mathrm{K}$ blackbody while the spectrun' of the visual Iuminosities corresponds to an F5 star (Crampton et al. 1975) and 
the light from the visual component is highly polarized. This observational evidence has been incorporated into a model which has the following structure: an F5 supergiant star is embedded in a dust torus. The torus is optically thick, completely obscuring direct observation of the central star. Light from the star scatters from dust grains in optically thin regions above and below the torus and is eventually seen by earth-based observers. Eanthbound observers see light that is scattered at 90 degrees from the original direction so that it is highly polarized. Also here it is assumed that the grains are small compared to the visual wavelengths. Unfortunately, nothing in the infrared or visual spectra gives a clue to the composition of the grains.

A nova is an event in which a star throws off a thin shell of matter of high density which moves with a high velocity sometimes as large as $1000 \mathrm{~km} / \mathrm{sec}$. This is accompanied by a spectacular rise in the Iuminosity of the star. At the time of initial ejection, the energy spectrum of the shell is that of a hot blackbody which has a temperature range from $5000^{\circ} \mathrm{K}$ to $10000^{\circ} \mathrm{K}$. As the dust shell expands the matter cools and in some cases dust grains condense. The condensation of dust grains is signaled by an 
increase in infrared flux from the object and a simultaneous drop in the visual flux. It is thought that the dust condensation process is very rapid, taking only a few weeks. For Nova Serpentis, most of the luminosity of the object was emitted in the infrared after dust condensation occurred (Hyland and Neugebauer 1970).

Infrared observations of some novae suggest that the flux comes from the expanding shell which cools as it expands. In contrast to this, Nova Vulpeculae 1976 has displayed a blackbody infrared spectrum that has remained at nearly constant temperature for several months (Ney 1977b). During this time the infrared luminosity has been decreasing and Ney has postulated that the infrared radiation is coming from the region of grain formation which is moving closer in to the star as the stellar luminosity decreases. For some novae in which dust condensations occurs, no silicate features appear presumably because the dust shells are composed principally of nonsilicates. This is apparently true for Nova Vulpeculae 1976 (Ney 1977b). However for the case of Eta Carinae, a nova which burst in the mid-19th century and around which there is now a very lange cool optically thin dust shell, broadband photometry has found a loum feature. 
The grains are somewhat hotter than blackbodies at the same distance from the central star, suggesting that the particles are small compared to the infrared wavelengths. The angular size of Eta Carina is wavelength dependent having approximately 3 times the angular siże at $10 \mu \mathrm{m}$ as it does at $2 \mu \mathrm{m}$. This is consistent with the idea that grains closer in to the stan are hotter and therefore radiate more strongly at shorter wavelengths (Gehrz et aI. 1973).

VI. THE IOHM FEATURE IN ABSORPTION A lange variety of objects in our galaxy display the 10um feature in absorption. Several of these have infrared spectra similar to that of the BecklinNeugebauen-Kleinmann-Low (BNKL) complex in the Orion Nebula (Gillett and Forrest 1973) which is shown in Figure 5. Roughly, the spectrum of the BNKL source appears as a themal continuum with two absorption features, one at approximately $3.1 \mu \mathrm{m}$ and the other at approximately $9.8 \mu \mathrm{m}$. The feature at $3.1 \mu \mathrm{m}$ is attributed to an $0-H$ vibrational band in water ice while the $9.8 \mu \mathrm{m}$ feature has the earmarks of an si-0 stretching mode due to silicates. The feature is smooth; i.e., no structure is seen, and the position of the peak agrees quite well with emission peaks seen in the spectra of $M$ stars. Comparison of the 


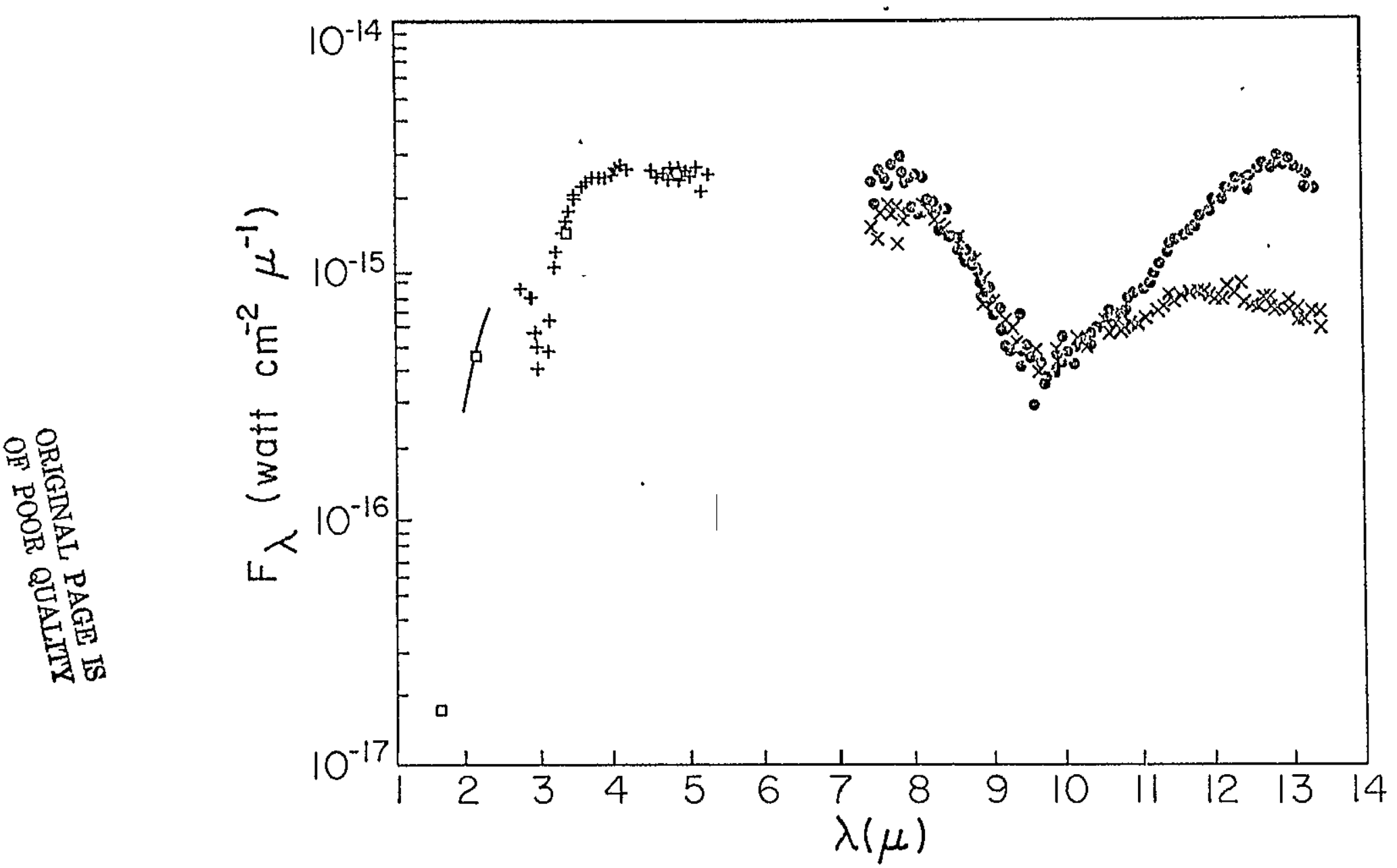

Fig. 5. Spectral observations of the $\mathrm{BN} \cdot \mathrm{KL}$ complex. $\mathrm{X}=\mathrm{BN} \quad \cdot=\mathrm{BN}+\mathrm{KL}$ From Gillett, F.C. and Forrest, W.J. 1973, Ap.J. 179, 483. 
infrared excess of certan $M$ stars to the absorption spectrum of the Becklin-Neugebauer object shows reasonably good agreement, indicating a strong similarity in composition between the dust grains in $M$ stars and the dust in the BNKL complex (Gillett and Forrest 1973). Generally speaking, the 10um absorption feature is due to cool silicate dust along the Iine of sight between an infrared source and an observer. The BNKL complex has two components, the Becklin-Neugebauer point source (BN) and a nearby extended source discovered by Kleinman and Low (KL). The extended KL source has a brightness temperature of about $70^{\circ} \mathrm{K}$ and is thought to be a collapsing cloud of gas and dust. The exact nature of the BN point 'source is unknown. Some research workers have. modeled it as a protostar, an intrinsically cool object, with intervening cold dust of the same composition which produces the loum absorption feature.

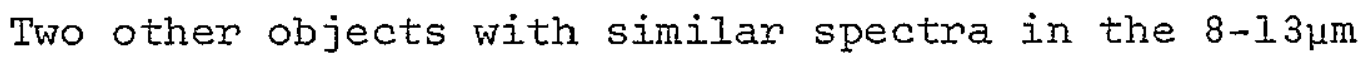
region are W3-IRS 5 and AFCRL No. 809-2992 (Aiken and Jones 1973; Merrill and Soifer 1974). For these objects the model of a massive protostar embedded within an extended dust cloud seems eppropriate. Merrill and Soifer (1974) have constructed a spectrum consisting of a blackbody source with emission from 
dust as seen in the Trapezium absorbed by cold intervening material of the same type. The fit of this model spectrum to the AFCRL 809-2992 spectrum is quite good, illustrating the similarity between dust seen in emission and dust seen in absorption in these two objects.

- Woolf (1973a) has reported the spectrum of the infrared core of the galactic center. The smooth absorption curve peaks at $9.7 \mu \mathrm{m}$ giving evidence of silicate dust toward the center of our galaxy similar to that seen in Orion. A heterogeneous group of infrared objects,was observed by.Merrill, Russell and Soifer (1976). With the exception of one, all of these objects are embedded in or are behind molecular clouds. They show absorption features at $3.1 \mu m$ and 9.7um due to ice and silicate grains in the molecular clouds which surround them. One of the objects shows no ice absorption but does have a weak absorption feature near $10 \mu \mathrm{m}$ and $i t$ is thought that this spectrum is due to dust in the unshielded interstellar medium, an environment not favorable to growth of ice. Jones and Merrill (1976) have assembled the infrared spectra of a number of oxygen-rich stars which have circumstellar dust shells of differing optical depths. The suggestion is that as the optical 
depth of a circumstellar dust shell changes from being optically thin to being optically thick, the $10 \mu \mathrm{m}$ feature changes from an emission feature to an absorption feature. They conclude that a circumstellar shell containing silicate grains will show the silicate feature in absorption if the shell's optical depth at loum is greater than 1 and the dust temperature is colder than $250^{\circ} \mathrm{K}$, assuming a latetype supergiant star as the central object.

The maser source 0 H23I. $8+4.2 \equiv 0$ H0739-14 has been associated with a strong extended infrared source located at the $\mathrm{OH}$ position. The spectrum shows strong absorption near $3.1 \mu \mathrm{m}$ and in the $8-14 \mu \mathrm{m}$ range. This object is well out of the galactic plane; Gillett and Soifer (1976) have reasoned that absorption is due to a large column density of material local to the source. The Ioum absorption feature is peculiar. It shows two peaks, one near $9.5 \mu \mathrm{m}$ and the other at 10.4um, instead of the usual single peak at or near 9.7um. The feature is also broader and shifted to longer wavelengths.

\section{INTERSTELLAR EXTINCTION}

Trumpler (1930) demonstrated the existence of a general interstellar absorption. He found that within a given class of galactic clusters an extinction of 
approximately $I$ mag/Kpc in the visual was needed to avoid the unlikely conclusion that there was a systematic increase of cluster diameter with distance from the observer. A similar conclusion was reached earlier by Struve (1847) using a method of star counts in which he compared the number of stars brighter than a given magnitude in relatively clear and in obscured parts of the sky. Stebbins, Huffer and Whitford (I939) measured the UBV magnitudes of B stars. These measurements indicated that starlight was reddened due to interstellar absorption and that the extinction in magnitudes varied approximately as $1 / \lambda$ over the UBV wavelength range.

An important research result related to the phenomenon of interstellar absorption was obtained by Dort (1932) who estimated the normal component of the galactic gravitational field by analyzing stellar motions at right angles to the galactic plane. Using this estimate of the gravitational field he calculared a value of approximately $3 \times 10^{-24} \mathrm{gm} /(\mathrm{cm})^{3}$ as an upper limit to the density of interstellar matter in the form of gas and dust.

Various ideas concerning the composition and sizes of interstellar grains have been proposed. Among these are metallic grains, graphite grains, 
dielectric grains, graphite and/or dielectric gains with ice mantles. Recently Ney and Merrill (1976) have argued against metallic particles in cometary grains based on forward scattering observations of Comet West.

An important source of information about interstellar dust is the extinction curve which is usually plotted as the normalized extinction $E(\lambda-V) / E(B-V)$ versus $I / \lambda(\mu \mathrm{m})^{-I}$. Such curves have been reported by Bless and Savage (1972) based on $\mathrm{OAO}-2$ and other data. Extinction curves taken from papers by Nandy and by Stebbins and Whitford are shown in the book by Wickramasinghe (1967). Inspection of these curves shows that the $\lambda^{-1}$ extinction law is approximately true for a range of $I / \lambda$ from $I$ to 4 . There is a prominent bump on the extinction curve at $I / \lambda=4.55$ or $\lambda=.22 \mu \mathrm{m}$. This was predicted by several research workers as a signature of graphite and is considered as one of the main bits of evidence for the existence of graphite in interstellar grains (Stecher and Donn 1965). Gilra (1971) has shown that the extinction bump can be fitted using graphite particles which are $\sim 0.025 \mu \mathrm{m}$ in radius and that a satisfactory fit to the extinction of the star. $\zeta \mathrm{Oph}$, taken from OAO-A2 observations, for values of $I / \lambda$ from $I$ to 9 can be obtained 
by a combination of graphite, silicon carbide and silicate grains. The silicates are the chief contributors in the far ultra-violet region. Huffman and Stapp (1971) have argued that the extinction curve of $\zeta$ oph can be fitted using a bi-modal distribution of silicate grains with no graphite component at all, but Ney et al. (1973) have questioned this interpretation of the $2200 \AA$ feature as being due to silicates, pointing out that their observations of emission nebulae around Orion $O$ and $B$ stars show the silicate emission feature while the OAO date of Bless and Savage (1972) show a relatively weak ultraviolet extinction peak for these objects.

Several objects (e.g., the BNKL complex and AFCRI No. 809-2992) which show the 10um silicate feature in absorption also show a $3.1 \mu \mathrm{m}$ absorption feature attributed to small ice grains. For these objects absorption is assumed to take place in cool extended clouds which surround them; Merrill and Soifer (1974) , have estimated the mass ratio of ice to silicates in the region surrounding the AFCRL object to be approximately 0.1. Also for the group of objects observed by Merrill, Russell and Soifer (1976c) which shows both ice and silicate absorption in extended molecular clouds the mass ratio of ice to silicates 
varied from .02 to .2. This set of observations also shows that abundance of ice in molecular clouds is greater than the abundance in regions of the interstellar medium away from such clouds. The ices are thought to be confined to molecular cloud regions and are probably a minor constituent of the general interstellar medium. A detailed comparison of the observed ice absorption profiles to the calculated profiles of water ice and solid $\mathrm{NH}_{3}$ by Merrill et al. (1976c) shows that a range of sizes in grains of solid $\mathrm{H}_{2} \mathrm{O}$ near. Ium, with some $\mathrm{NH}_{3}$ mixed in, can give a reasonable fit to the observed absorption features. Most estinates of the relative amounts of ice and silicates in interstellar clouds assume the ice and silicates exist as separate particles; however, some investigators have calculated the absorption properties of silicate core-ice mantle particles. Aannestad (1975) has considered silicate cores composed of either quartz, olivine, or lunar material and found a slight broadening and a shift of absorption features toward Ionger wavelengths by .1-.3um due to an ice mantle. He found that a calculated absorption spectrum of olivine core-ice mantle particles fit the observed strengths of the ice and silicate absorption peaks in the BN spectrum using a 
mantle-to-core volume ratio of 1.3. The use of olivine as the core material gives structure in Aannestad's calculated loum feature that is not seen in the $B N$ spectrum. The volume ratio of 1.3 gives an ice-to-silicate mass ratio of approximately .4 which is somewhat higher than the range of values given by Merrill et al. (1976c) for ice-to-silicate ratios in the group of molecular clouds they studied. Dorschner et al. (1976) have calculated equivalent widths for the 10um spectral absorption features of the BN object and the galactic center. Using an experimentally derived curve of growth of olivine particles, they derive mass densities averaged along the line of sight to the galactic center and the BN source of $3 \times 10^{-26} \mathrm{gm} /(\mathrm{cm})^{3}$ and $4 \times 10^{-25} \mathrm{gm} /(\mathrm{cm})^{3}$ respectively. These values are compatible with the upper limit of interstellar matter given by oort (1932).

Major components of the interstellar medium are silicates and graphite, because of their manufacture and dispersal into space by $M$ stars and $C$ stars. other components are SiC and ice (found primarily in interstellar clouds).

In summary, evidence for graphite and silicates in submicron form in the interstellar medium is seen 
in the bump on the extinction curve at $2200 \AA$ and in the 10um absorption feature. Gilra (1971) has used a three component grain mixture consisting of graphite, silicon carbide and meteoritic silicates to fit the interstellar extinction curve. Water ice is thought to exist in molecular clouds but certain observations suggest that the abundance of ice in the unshielded interstellar medium is much less than in molecular clouds. A rough estimate of the mass ratio of dust to gas in the interstellar medium is 1:100 (Woolf 1973a).

\section{COMETS}

Comet Ikeya-Seki (1965f) was observed at inirnared wavelengths by Becklin and Westfall (1966); their observations were the first ones of a comet in this wavelength range. Working at wavelengths from $1.65 \mu \mathrm{m}$ to Ioum they detected Ilux from the head and tail of the comet. The color temperatures indicated that the emitting material was hotter than a blackbody at the same distance from the sun. It was assumed that the thermal energy was coming from particles released from the comet's nucleus-particles which were more efficient absorbing visual radiation than emitting in the infrared. In this way the higher temperature of the emitting matter could be explained. Iron was 
postulated as the grain material because iron lines had been found in the comet's spectrum and because the emissivity of iron as a function of wavelength has approximately the correct behavior. The head of the comet was observed to have an optical depth of $\sim 10^{-3}$.

Maas, Ney and Woolf (1970) reported observations of Comet Bennett using a broadband photometric system. These observations followed closely the initial work on the infrared spectra of cool M stars by Gillett, Low and Stein (1968) and Woolf and Ney (1969). It was thought that silicates would be present in comets since silicates are a major component of the carbonaceous chondrite meteorites which, along with comets, are believed to be remnants of primitive solar material. Comet Bennett's infrared spectrum was found to be that of a thermal continuum with a strong emission feature superimposed near 10um. As was the case for Comet Ikeya-Seki the matter was hotter than a blackbody at the same distance from the sun and the object was optically thin (i.e., $\tau \sim 10^{-4}$ ). Woolf and Ney proposed two types of grains to account for the comet's spectrum. One component would consist of particles which are gray or black in the 2-5um region and which are small with respect to the infrared wave- 
lengths being emitted. These particles would produce a thermal spectrum because they have little or no variation of emissivity with wavelength and would be hotter than a blackbody at that distance from the sun because they are not efficient radiators of infrared energy and must radiate at a higher temperature to achieve energy balance. A graphite-iron mixture could possibly produce this part of the spectrum. For the 8-20um region, the second component must have a strong variation of emissivicy as a function of wavelength and the grains individually must be optically thin or else their emissivity variation cannot be seen. Maas et al. (1970) proposed silicates similan to those found in the carbonaceous chondrites as being the second type of material present in cometary dust; they estimated the silicate mass in the dust to be several times greater than the blackbody mass.

Comet Kohoutek was observed in December 1973 and January 1974 by Ney (1974b) using broadband photometry $(\Delta \lambda / \lambda \sim .1)$ and in December 1973 by Merrill (1974) using intermediate resolution spectrophotometry $(\Delta \lambda / \lambda \sim .01)$. Spectra of Comet Kohoutek taken by Ney are shown in Fig. 6 . The plot here is $\lambda F_{\lambda}$ versus $\lambda$ on a $\log -\log$ scale where $F_{\lambda}$ is the energy flux from the comet in watts $/ \mathrm{cm}^{2} \cdot \mu \mathrm{m}$ and $\lambda$ is the effective 


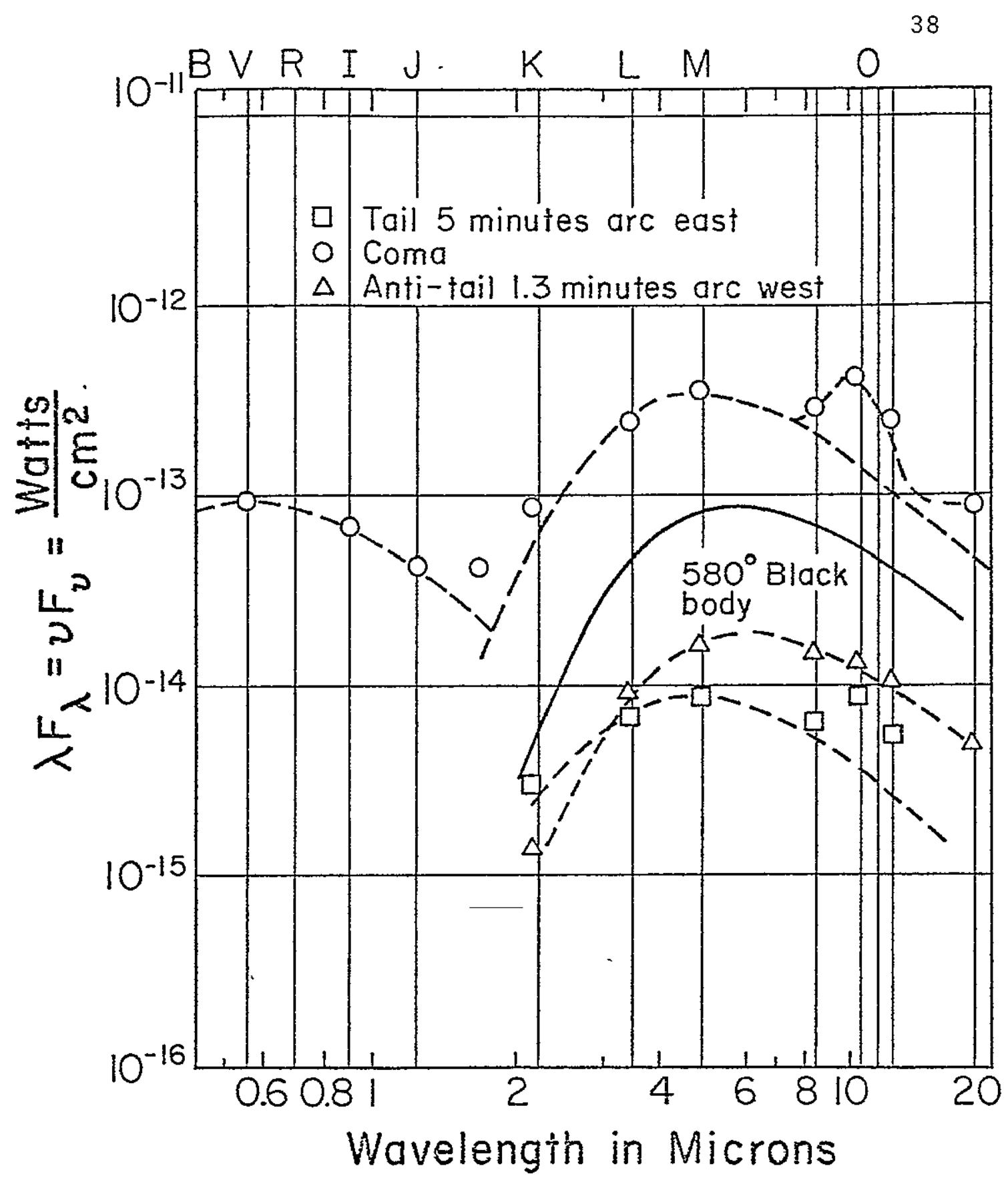

Fig. 6. Broadband photometry of Comet Kohoutek measured on 1974 January 1.7. From Ney, E.P. 1974, Ap.J. (Letters) 189, L141. 
wavelength of observation in $\mu \mathrm{m}$. On such a curve a blackbody has a fixed shape. The relation between the wavelength of the maximum value of $\lambda B_{\lambda}$, where $B_{\lambda}$ is the blackbody flux, and the blackbody temperature $T$ is given by

$$
\lambda_{\mathrm{m}}=3670 / \mathrm{T}
$$

with $T$ in Kelvin and $\lambda^{\prime}$ in $\mu \mathrm{m}$. The spectrum of the coma of Comet Kohoutek shows the colors of reflected sunlight in the visual wavelength region and a thermal continuum with a $10 \mu \mathrm{m}$ feature superimposed on it in the infrared. As was the case for comets Ikeya and Bennett, the optically thin dust grains were hotter than a blackbody at that distance from the sun. Ney (1974b) reasoned that Rayleigh scattering would suppress the short wavelength intensities if the grain sizes were much smaller than the visual wavelengths and gave a lower limit to particle size of approximately $0.2 \mu \mathrm{m}$. This size estimate agrees fairly well with the results of Rayleigh scattering experiments carried out by Pfund (1934) who measured the transmission of visual and near infrared energy through zinc-oxides dispersed in rubber. He found that transmission agreed with theoretical predictions based on Rayleigh scattering if particle diameters 
were less than .I um but departures from theory were seen for particles with diameters larger than . $2 \mu \mathrm{m}$. Since the silicate particles show a variation of emissivity with wavelength, their optical depth at the center of the absorption band cannot be much greater than unity. Typical mass absorption coefficients for silicates are around $5000 \mathrm{~cm}^{2} / \mathrm{gm}$ and a corresponding grain dimension for unity optical depth, if a density of $2 \mathrm{gm} / \mathrm{cm}^{2}$ is assumed, is $I \mu \mathrm{m}$. Therefore, it is thought that silicates in this size range give rise to the $10 \mu \mathrm{m}$ feature while graphite particles of approximately the same size are responsible for the infrared thermal continuum in the 2-8um region. Using the ratio of power radiated toward the earth at infrared wavelengths to the power reflected toward the earth at visual wavelengths, Ney (1974) calculated the cometary albedo to be 0.2 . This value of albedo is consistent with that expected for dielectric grains but unfortunately does not eliminate other possibilities. Scattering function measurements of comet West were more discriminating. The silicate feature is also seen in the spectrum of grains in the tail of Comet Kohoutek, which are at approximately the same temperature. as the grains in the coma. Obtaining the spectra of 
grains in the anti-tail of Comet Kohoutek was an observational first; the discovery was made that this spectrum displayed no silicate feature and was much closer in color temperature to a blackbody (Ney 1974a). It is believed that this result was due to the presence in the anti-tail of grains which were larger than those found in the coma and tail. Sekanina (1974) has shown that the so-called anti-tail is a projection effect which is seen when the earth is in proper position with respect to the sun and comet. Langer particles, for which the ratio of radiation pressure to gravitational attraction is small, move in independent orbits around the sun, being emitted from the comet several weeks before the time of observation, and are seen by observers in the plane of the comet's orbit as a sunward spike. Particles in the tail have sizes such that the ratio of radiation pressure force to gravitation force is larger and they are blown more radially outward from the sun. This analysis.supports the view that the absence of a loum feature in the anti-tail is a grain size effect. Ney (1974b) was able to observe this comet on several days near the time of passage through perihelion and found the color temperature of the infrared part of the curve rose to $\sim 950^{\circ} \mathrm{K}$. 
Post perihelion observation showed the presence still of the loum feature, suggesting that the emissivity of the grains was not changed by heating and that the grains are able to withstand temperatures in the $900^{\circ} \mathrm{K}$ range without drastic alterations in structure. To date the only intermediate resolution spectra of a comet in the loum region is the one taken by Merrill (1974). See Figure 13. Using a filter wheel for which $\Delta \lambda / \lambda \cong .015$, Merrill observed Comet Kohoutek in the 8-13um wavelength range on 21.7 December 1973, approximately 7 days before perihelion. There was a gap in Merrill's data near $10.5 \mu \mathrm{m}$ due to technical difficulty. A spectrum representing the excess in the region near I0um was gotten by subtracting a $600^{\circ} \mathrm{K}$ thermal continuum from the cometary spectrum, $600^{\circ} \mathrm{K}$ being the appropriate color temperature of the comet at that point in its journey around the sun. The cometary emission feature shows no structure. Comet West came under observation in the infrared in January 1976 and become a member of the "10um feature club" along with Comets Bennett, Kohoutek and - Bradfield. The silicate feature was seen in its spectra superimposed on a thermal continuum from February through April of that year at which time it became too dim to be seen in the infrared. The 
visual part of its spectrum was due to reflected sunlight. Because Comet West passed between the earth and the sun, a unique opportunity was presented to study the light scattering of the dust as a function of scattering angle. It was unique because the albedo deteminations for Comets Kohoutek and Bradfield were made for scattering angles near 90 degrees but for Comet West observations were made for forward scattering angles ranging from 34 degrees to 150 degrees. Near perihelion Comet West Iragmented into four pieces and the increased total nuclear surface area produced an increase in the intrinsic brightness of the coma. The scattering phase function of the comet's dust grains is proportional to the ratio of the reflected energy to the emitted energy of the dust which Ney and Merrill (1976) assumed was equal to the ratio of the visual energy to the infrared energy. They compared the observed scattering phase function of Comet West's grains to the scattering phase function expected for Rayleigh scattering, conducting grains and dielectric grains. The strong forward scattering of comet West excludes Rayleigh scattering, which is isotropic, as a mechanism. However it was found that the calculated scattering phase function for dielectric materials (such as silicates) with $1 \leq \frac{a}{\bar{\lambda}}<2$ gives a good fit to. 
the observational data. The grain radius is denoted by $a$ and $\lambda$ is in the visual wavelength range. By integrating the observed ratio of reflected to emitted flux over all solid angles, Ney and Merrill (1976) estimated the value of the Bond albedo, A, to be in the range $0.3-0.5$, indicating that the grains are fairly good absorbers of visual radiation. Since the cometary loum feature resembles strongly the $10 \mu \mathrm{m}$ feature seen in other astrophysical objects, it is assumed that there is a similarity in composition and structure between the comet grains and grains which are thought to condense in the atmospheres of cool stars. Therefore the study of cometary spectra is valuable in trying to understand the nature of grains forned in other astrophysical environments. As Ney (1977a) has pointed out, the light from comets can be studied without being contaminated by the direct light of the illuminating star; also the grains in the coma of a comet are at a uniform temperature determined by thein distance from the sun in contrast to the varying temperatures that probably exist for grains in circumstellar shells of stars. Comets are believed to be remnants of primitive solar nebula material and therefore should have some resemblance to Type 1 carbonaceous chondrites. 


\section{LABORATORY MEASUREIIENTS OF SILICATES}

Transmission and reflection properties of quartz in the 4.5-10.5um range were published by Pfund (1933). He found a transmission maximum in samples of powder film of $5 \mu \mathrm{m}$ crystalline quartz at $7.3 \mu \mathrm{m}$ and a reflection maxinum of a polished quartz plate in a band from 8.5 to $9.3 \mu \mathrm{m}$. These maxima are related to the Christiansen effect and the so-called reststrahlen features. Early papers by Hunt, Wisherd-and Bonham (1950) and Launer (1952) have been usefuI to astrophysicists because they contain infrared absorption spectra of a large number of minerals, rocks and inorganic compounds. More recent publications which also Iist a lange number of infrared spectra of minerals and compounds are those by Hunt and Salisbury (1974) and by Nyquist and Kagel (1971). The reference by Hunt and Salisbury is particularly useful because it gives a qualitative discussion of infrared absorption processes in silicates and an explanation of rock classification schemes used by geologists. A report by Lyon (1964) contains a large number of spectra of minerals and rocks and was prepared with an astrophysical objective in mind; namely, the use of infrared spectrophotometry for compositional analysis of lunar and planetary soils. 
A great deal of laboratory work has been done acquiring infrared emission spectra of silicates under simulated Iunar conditions (Logan and Hunt 1970; Logan, Hunt, Blasamo and Salibury 1972). In general this involves viewing optically thick samples for which an emittance maximum occurs at the Christiansen frequency. At this wavelength, which occurs on the short wavelength side of the Si-o stretching mode absorption band, the real part of the complex refractive index is equal to unity and the imaginary part is small since there is little absorption. This results in maximum transmission of the surface layer of the sample because scattering is at a minimum and absorption is small, giving an emission peak for the sample as a whole.

of particular importance for the research effort reported in this thesis have been the studies by several workers devoted to gaining information about silicatés as it relates to understanding properties of astrophysical grains. Steyer et al. (1974) and Day et al. (1974) have measured the extinction of quartz and other silicates in submicron form and have concluded that earlier estimates of the mass absorption coefficients of silicates in the loum absorption band are too low for the following reason: 
earlier quantitative work used particles in the $2-4 \mu \mathrm{m}$ diameter range and for particles of this size only a fraction of the mass is needed to comletely absorb the resonance wavelengths. The rest of the mass does not contribute to the absorption but serves only to lower the calculated value of the mass absorption coefficient. A true measure of the mass absorption coefficient is obtained only by using smaller particles, Day et al. (1974) also concluded that for silicates the extinction at visual wavelengths does not become much greater than the absorption in the $10 \mathrm{~m}$ region. Since the average observed ratio of visible to infrared $10 \mu \mathrm{m}$ extinction is $50: 1$, accord. ing to Woolf (1973a), either astrophysical silicates have a different mass absorption coefficient (and therefore differ structurally from terrestrial silicates) or other grain species are present in interstellar space to provide visual extinction.

The real and imaginary parts of the complex refractive index have been reported in the infrared for quartz and olivine (Steyer et al. 1974; Steyer 1974). This is useful because Mie theory calculations may be carried out for small grains of these materials. The Mie theory formulation arises from a rigorous solution of Maxwell's equations subject to the 
appropriate boundary conditions on the spherical surface. This theory is used to describe the scattering and absorption of infrared radiation by small grains for which $\lambda$, the wavelength, and the particle's diameter are roughly equal. The uses of Mie theory in astrophysical problems are given in books by Wickramasinghe (1967) and Van de HuIst (1957). Comparisons of calculated values of extinction of fine particle silicates to measured values of extinction have shown fairly good agreement between theory and experiment as far as the position of peak extinction is concerned; however, the calculated absorption feature is always narrow compared to the experimental one. This is due in part to a difference between the sizes and shapes in the experimental sample and the assumed sizes and shapes.used in calculations, although the complete explanation for this discrepancy is not known (Day 1975).

Day (1974) has prepared the magnesium end member of the olivine series (forsterite) by mixing sodium orthosilicate and magnesium chloride together in water in correct proportions to achieve the following reaction:

$$
\mathrm{Na}_{4} \mathrm{SiO}_{4}+2 \mathrm{MgCl}_{2} \rightarrow 2 \mathrm{MgO}+\mathrm{SiO}_{2}+4 \mathrm{NaCl}
$$


The oxides precipitated as a gel and the salt was removed by washing. In its low temperature form the synthetic silicate shows a smooth loum absorption feature resembling somewhat the astrophysical 10um absorption feature which does not show the structure characteristic of terrestrial silicates in the I0um region. The low temperature material showed no $x$-ray diffraction lines but after being heated to $750^{\circ} \mathrm{C}$, the full range of forsterite lines was seen and the infrared spectrum was identical to that of forsterite $\left(\mathrm{Mg}_{2} \mathrm{SiO}_{4}\right)$. Heating therefore caused a transition from an amorphous state to a crystalline phase. In its Low temperature form Day noted that the amorphous silicate gives a reasonable fit to the absorption feature of the Becklin-Neugebauer object in Orion and also bears a strong resemblance to the absorption spectrum of the Murchison meteorite, a Type 2 carbonaceous chondrite, although the Murchison spectrum peaks at approximately $10.3 \mu \mathrm{m}$ while the synthetic silicate spectrum peaks near $9.8 \mu \mathrm{m}$. The appearance of structure in the spectra of this material when heated strongly suggests that if it is a major component of silicate grains it must exist in an unheated form. Measurements of the mass absorption coefficient of amorphous silicates by Day (1976) yield 
values in the range from $3000-5000 \mathrm{~cm}^{2} / \mathrm{gm}$. These are lower by a factor of 2 or so than the values of a pure crystalline silicate. Gillett et al. (1975) have indicated that only 5 of the 10 magnitudes of visual extinction in the spectrum of the highly reddened star VI Cyg No. 12 could be produced by the amount of pure silicates needed to produce the observed $10 \mu \mathrm{m}$ absorption, assuming a silicate opacity of 5000 $\mathrm{cm}^{2} / \mathrm{gm}$, and therefore another grain component is needed. If, however, the opacity of astrophysical grains is lower, then smaller amounts of additional grain species are needed to produce the visual extinction, and the measurements by Day show a lower opacity.for the synthetic amorphous materials.

The possibility that hydrous layer lattice silicates are a component of grains in interstellar space and in circumstellar shells of stars has been explored by Zaikowski, Knacke and Porco (1975a) and Zaikowski and Porco (1975b). They along with others have shown that the terrestrial silicates olivine and enstatite have a great deal of structure in their loum absorption bands unlike the smooth feature that occurs in astrophysical spectra. Since the Types 1 and 2 carbonaceous chondrites are thought to contain some material which appears to be primitive or 
unaltered, they investigated terrestrial silicates of similar structure. The $\mathrm{Cl}$ meteorites are composed primarily of hydrous layer lattice silicates or phyllosilicates. As the names imply, these silicates are made up of layers of silicate tetrahedra; occasionally $\mathrm{Al}$ and $\mathrm{Mg}$ may substitute for $\mathrm{Si}$ in the $\mathrm{SiO}_{4}$ structure. Varying amounts of water can exist between the silicate layers. Absorption spectra of Orgueil ( $\mathrm{Cl}$ ), Cold Bokkeveld (C2), and Murray (C2) strongly resemble astrophysical absorption spectra (Zaikowski and Porco 1975). Of these three Murray has the highest percentage of olivine and pyroxene, the high temperature condensates.

Zaikowski et al. (I975a) found that several terrestrial phyllosilicates (e.g., montmorillonite) have absorption features near $9.8 \mu \mathrm{m}$, that their spectra for the most part show less structure than the olivine or enstatite spectra. In this respect they give a better match to the astrophysical absorption spectra than do olivine or enstatite. However, the absorption features of phyllosilicates are narrow compared to the astrophysical feature.

One of the few experimental studies that has artempted to model condensation in a cool stellar or primordial atmosphere is the one conducted by Meyer 
(1970). He produced a partially ionized vapor containing $\mathrm{Ar}, \mathrm{O}, \mathrm{Si}, \mathrm{Mg}, \mathrm{Fe}, \mathrm{Al}, \mathrm{Ca}$, and $\mathrm{H}$ and obtained thin films of crystalline and amorphous silicates. In some instances, he used relatively cool substrates thus stimulating in one way the non-equilibrium condensation process envisioned by Arrhenius and Alfven in which grain condensates are cooler than the surrounding gas and ions. By allowing some water vapor into the vacuum system Meyer formed silicates resembling the matrix material of $\mathrm{Cl}$ meteorites. 
CHAPTER 2

LABORATORY INFRARED STUDIES OF SILICATES

The emphasis in this project was on simulation of cometary spectra because of the fact that comet grains are at a uniform temperature determined by their distance from the sun. The University of Minnesota laboratory experiment attempted to simulate infrared cometary spectra in the following way: optically thin samples of submicron particles were heated to temperatures typical of the cometary environment and viewed in emission against a lowtemperature background, using a laboratory setup which was almost identical to that of an infrared telescope in its operation.

\section{SAMPLE PREPARATION}

Grinding of materials was done using an agate mortar and pestle or a tungsten carbide wiggle-bug. Samples containing sub-micrometer particles were prepared by using a sedimentation technique in which finely ground material was dispersed in a liquid and allowed to settle for a certain time. A top layer of liquid (usually $I \mathrm{~cm}$ ) was drawn off and the particles from this layer were collected with the aid of a centrifuge. The uppen limit to the size of particles which were collected in this way was 
calculated using Stokes' Law (Orr and Dallavelle 1959) which can be written in the following form:

$$
t=\frac{18 \eta \times 10^{8}}{\left(\rho-\rho_{0}\right) g d^{2}(3600)}
$$

where $t=$ time in hours for a particle to fall $1 \mathrm{~cm}$ $\eta=$ viscosity of liquid in poise

$\mathrm{g}=980 \mathrm{~cm} / \mathrm{sec}^{2}$

$d=$ particle diameter in $\mu \mathrm{m}$ $\rho_{0}=$ density of liquid in $\mathrm{gm} /(\mathrm{cm})^{3}$ $\rho=$ particle density in $\mathrm{gm} /(\mathrm{cm})^{3}$.

A plot of this equation is shown in Figure 7 for the case of particles settling in acetone. At room temperature the viscosity and density of acetone are approximately .32 centipoise and $.8 \mathrm{gm} /(\mathrm{cm})^{3}$ respectively. From this plot it can be seen that over-night settling times in acetone should yield upper limits of $\sim 3 \mu \mathrm{m}$ in the top $1 \mathrm{~cm}$ layer of the liquid for materials with densities in the range from 2 to $3 \mathrm{gm} /(\mathrm{cm})^{3}$. Electron micrographs have been taken of some of the samples and show that the actual upper limits to the particle sizes were somewhat larger than the predicted values but most were less than $.5 \mu \mathrm{m}$ in 'diameter. Settling times of $1 \mathrm{hr} / \mathrm{cm}$ and $15 \mathrm{~min} / \mathrm{cm}$ were 


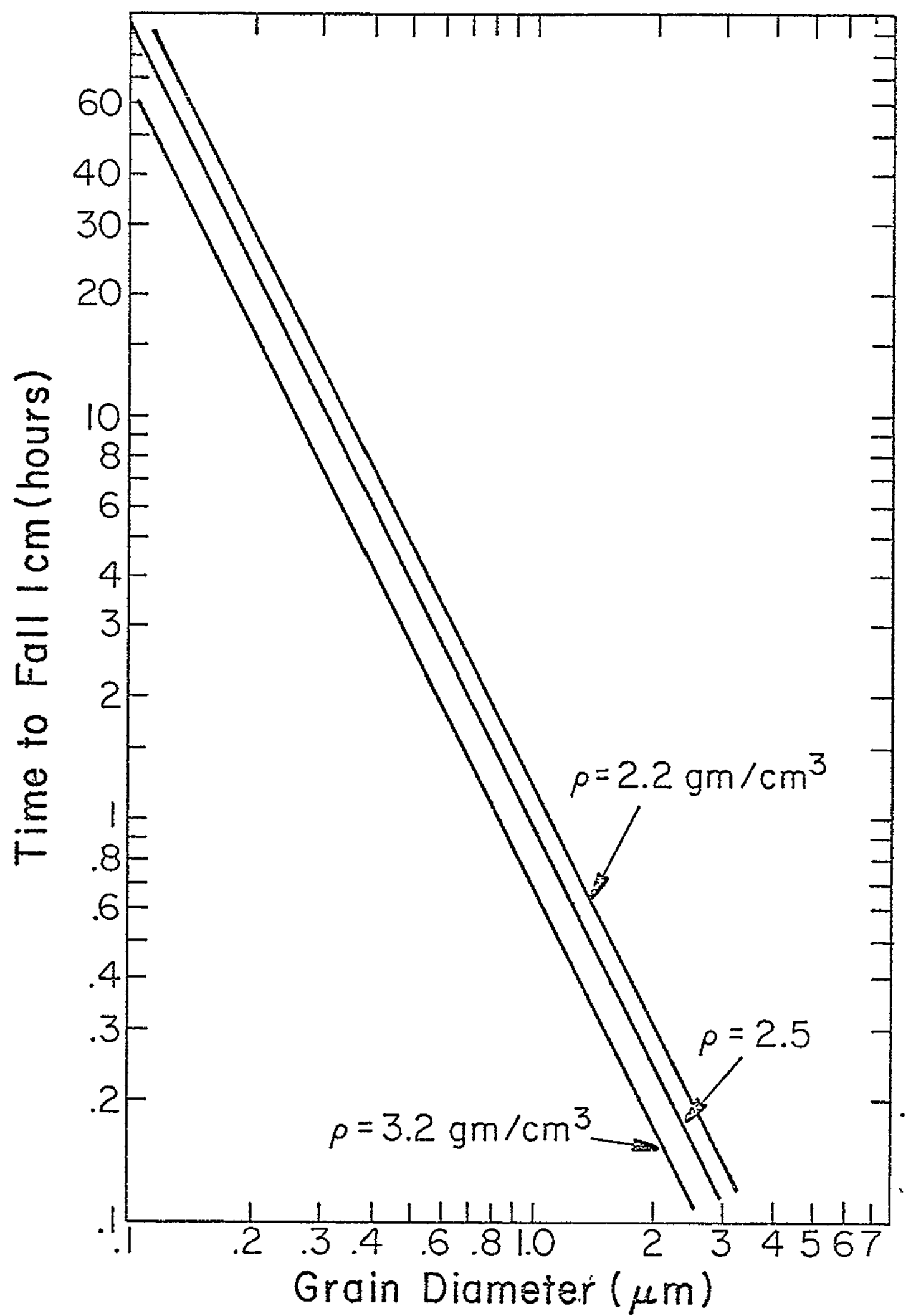

Fig. 7. Sedimentatıon time in acetone (Stokes' Law). 
used to produce samples in the approximate size ranges of $0-1 \mu m$ and $0-2 \mu m$ respectively. These larger upper limits were not checked with the electron microscope but were simply assumed to be correct based on the Stokes' Law calculations. Samples containing even larger size grains were prepared using a set of sieves which had square screen apertures of $74,53,37,25,16,10$, and $6 \mu \mathrm{m}$. Suitable low emissivity substrates were prepared by evaporating $2500 \AA$ aluminum thin films onto glass slides (Drummeter and Hass 1964). A substrate was then lowered into a test tube of acetone containing small particles, and some grains adhered to the metal film surface. The process of lowering the substrate into a tube of acetone in which the particles were dispersed was repeated until a satisfactory coverage of the thin film surface was obtained. The sample was dried between each dipping of the substrate in the acetone. Using these techniques, samples were prepared having small grains mostly one layer deep which covered $25 \%$ to $50 \%$ of the metal film surface.

\section{EXPERIMENTAL}

Figure 8 shows a schematic diagram of the experimental arrangements. The grain sample was mounted on a soldering iron heating element with a Dow-Corning ' 
heat sink compound. This compound served as an adhesive to hold the sample in place and provided good thermal contact between the heating element and the sample substrate. The heating element permitted a range of substrate temperatures up to $800^{\circ} \mathrm{K}$ to be used. All samples were heated in ain and unless otherwise noted a temperature of approximately $600^{\circ} \mathrm{K}$ was used. Blank substrate temperatures were measured using a copper-constantan thermocouple and a Keithley electrometer. A metal mirror with either a $1 \mathrm{~mm}$ hole or a $2 \mathrm{~mm}$ hole in its center was located $10 \mathrm{~cm}$ in front of the sample. Both sample and minror were tilted at 45 degrees so that the background radiation which they reflected into the system came from a liquid nitrogen tank which was located directly beneath the sample and metal mirror. A test stand was located approximately 15 meters away from the sample. The test stand which consisted of a primary mirror, a beam switching secondary and a mount for holding a nulti-filter dewar was almost identical in its operation to the telescope at the O'Brien observatory. The filters used in the laboratory experiment were centered at $2.2,3.5,4.8,8.5,10.6,12.5$, and $18 \mu \mathrm{m}$. They were identical to the ones used for astronomical 

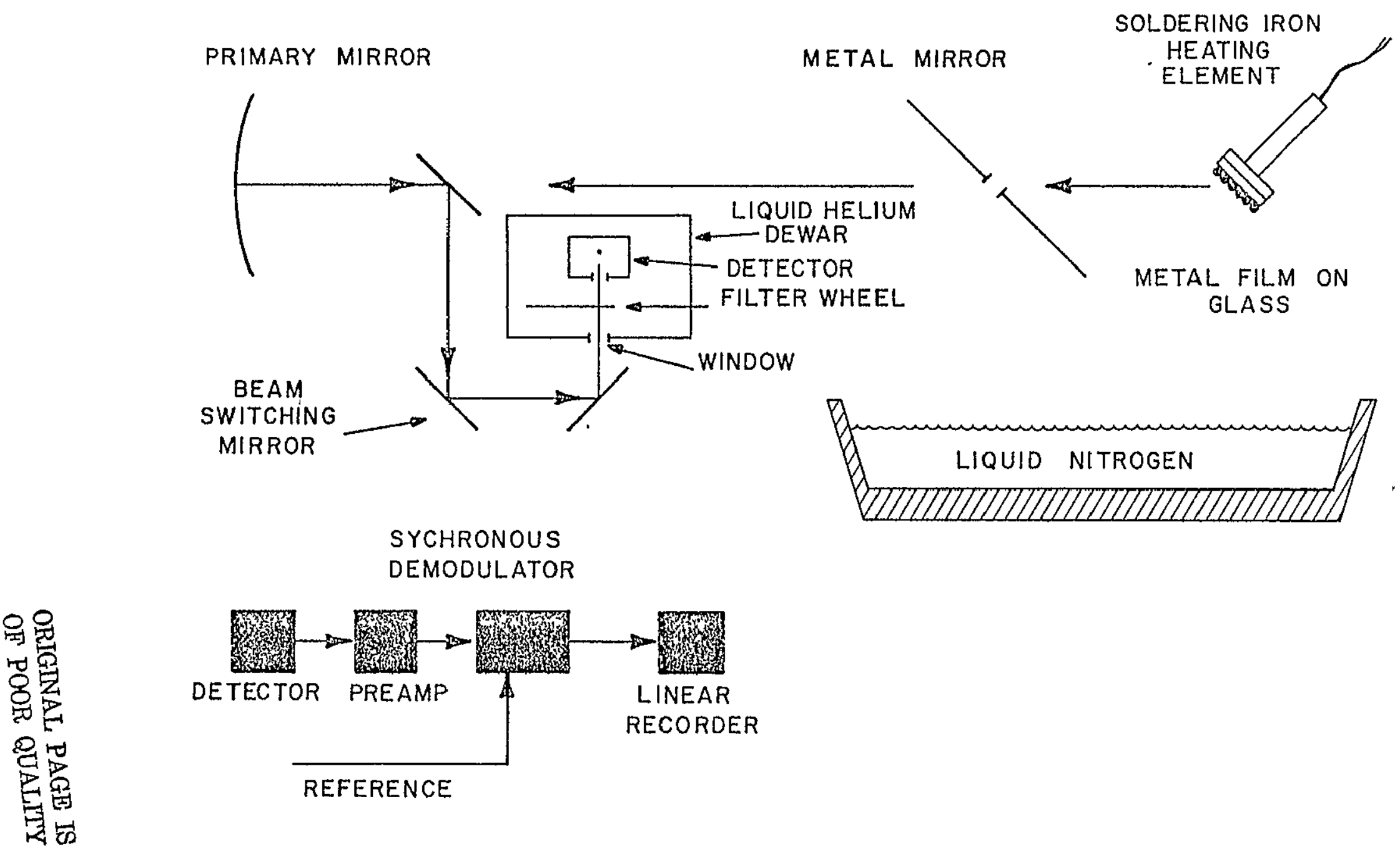

\section{DEMODULATOR}

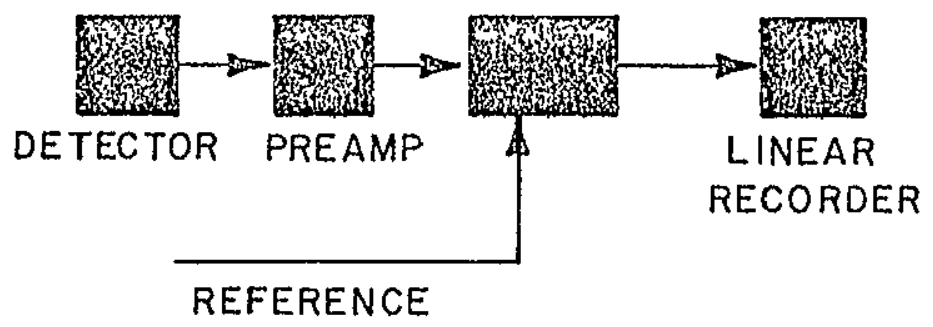

Fig, 8, Schematic diagram of test stand (Maasometerl and s.ample holder: 
observations for which the bandpasses are such that $\lambda / \Delta \lambda \approx 10$. After passing through a preamplifier, the detector signal was fed into a synchronous demodulator along with a reference signal from the beam switching mirror. The resulting d.c. output was displayed on a linear chart recorder. Additional information on the Minnesota infrared broadband photometry system can be found in the literature (Ney 1974b; Strecker and Ney 1974).

Room temperature absorption spectra of some samples were obtained by use of a Perkin Elmer Model 521 grating infrared spectrophotometer. The mirror substrates containing the grains were placed at the position of a small flat mirror in the sample beam of the instrument. These samples were the same ones which had been used. to obtain emission spectra.

\section{MATERIALS}

Spectra of 9 terrestrial silicates, I synthetic silicate, 6 meteorietic materials and 2 lunar soils were included in this study. Table 1 gives a list of samples used in this study and a brief description of each. The CIPW mineral norms which give a hypothetical mineralogy based on laboratory chemical analysis (expressed in terms of oxides) were supplied by Claus Shultz (see Appendix C). Descriptions of 
meteorites including chemical compositions are given by Wood (1963). Chemical analysis of lunar soils is given by Heiken (1974).

TABEE 1

SAMPLES USED IN THIS STUDY

MATERIAI

1) Terrestrial

Albite

Anorthite

Columbia River Basalt

Diabase

Dunite

\section{DESCRIPTION}

$\mathrm{NaAlSi}_{3} \mathrm{O}_{8} \mathrm{~A}$ plagioclase feldspar.

$\mathrm{CaAl}_{2} \mathrm{Si}_{2} \mathrm{O}_{8} \mathrm{~A}$ plagioclase feldspar.

This volcanic material consists of $28 \%$ albite, $18 \%$ anorthice, $9 \%$ quartz and $25 \%$ pyroxene in the form of diopside ( $\mathrm{CaMgSi}_{2} \mathrm{O}_{6}$ ) and hypersthene, (Mg, $\mathrm{Fe})_{2} \mathrm{Si}_{2} \mathrm{O}_{6}$, (CIPW mineral norms). The simple chain structure of pyroxenes can be represented by the general formula: $\mathrm{XY}\left(\mathrm{Si}_{2} \mathrm{O}_{6}\right)$.

An igneous rock. The principal minerals are albite $(20 \%)$, anorthite $(20 \%)$ and pyroxene (40\%). (CIPW mineral norms).

The majon phase is forsterite, $\mathrm{Mg}_{2} \mathrm{SiO}_{4}$, the magnesium end member of the olivine series. Serpentine, $\mathrm{Mg}_{6}\left(\mathrm{Si}_{4} \mathrm{O}_{10}\right)(\mathrm{OH})_{8}$, is also present. 
MATERIAL

Fayalite

SIag

Amorphous

magnesium

silicate

Quartz

2) Meteoritic

Orgueil

Ivuna

Murray

Allende
DESCRIPTION

$\mathrm{Fe}_{2} \mathrm{SiO}_{4}$ from Rockport, Massachusetts.

A fayalitic crystalline phase in a matrix of fine pyroxene crystallites. This material is from an ancient metallungical site at Limni, Cyprus (Cooke 1976).

A clay mineral composed of hydrous layer lattice silicates. (Zailowski et al. 1975a).

An amorphous material chemically similar to enstatite, $\mathrm{Mg}_{2} \mathrm{Si}_{2} \mathrm{O}_{6}$.

$\mathrm{SiO}_{2}$ in a framework silicate structure.

A Type I carbonaceous chondrite (CI) composed mainly of hydrous layer lattice silicates. The carbonaceous chrondrites contain the non-volatile elements in approximately solar proportions. (Wood 1963, 1968).

A Type I carbonaceous chondrite.

A Type 2 carbonaceous chrondrite (C2). It consists of hydrous layer lattice silicates, olivine $\left.(\mathrm{Mg}, \mathrm{Fe})_{2} \mathrm{SiO}_{4}\right)$ and

pyroxene. (Zaikowski et al. 1975a).

A Type 3 carbonaceous chrondrite (C3). Olivines and pyroxenes dominate the composition. (Wood 1963). 
MATERIAL

Abee

Juvinas
DESCRIPTION

An enstatite chondrite, The major minerals are enstatite, plagioclase feldspar and quartz (Wood 1963).

A calcium rich acondritic meteorite. It consists of $30 \%$ calcic plagioclase feldspar, $65 \%$ iron rich clinopyroxene and 5\% accessory minerals. (Duke and Silver 1967).

32 Lunar

Iunar soil 1553 I An immature mare basalt surface soil. Its normative composition is $26 \%$ plagioclase feldspar (mostly anorthite), 5\% orthoclase, $47 \%$ pyroxene and $10 \%$ olivine. (Heiken 1974, Adams 19762 .

Lunar soil 67701 An immature highland soil. Its nommative composition is $75 \%$ anorthite, $5 \%$ albite, $10 \%$ pyroxene and $8 \%$ olivine. (Heiken 1964, Adams 1976).

IV. LABORATORY BROADBAND SPECTRA

The results of viewing a sample under the conditions described in the Experimental section are shown in Figure 9. The plot is relative $\lambda F_{\lambda}$ versus $\lambda$ on a log-log graph. This plot is the same kind as was used in Figure $\sigma$ and $F_{\lambda}$ and $\lambda$ have the same definitions as were given in the section on comets.

- The top curve in Figure 9 is a DIackbody 
spectrum. Its verticle position has been chosen arbitrarily but the position of the peak of the spectrum has been placed on the horizontal scale at the wavelength corresponding to $550^{\circ} \mathrm{K}$, the temperature of the laboratory blackbody. The lower curves are spectra of fayalite and a blank aluminum substrate taken at a temperature of approximately $580^{\circ} \mathrm{K}$. At each of the wavelengths mentioned in the previous section, the brightness of the blank substrate or sample was calculated in magnitudes with respect to the laboratory calibration blackbody using the relation

$$
M_{1}-M_{2}=(-2.5) \log \left(V_{1} / V_{2}\right)
$$

where $V_{I}$ was the output voltage when the calibration blackbody was being viewed and $V_{2}$ was. the corresponding voltage of the blank substrate. A tabulation of voltage signals and magnitude calculations related to Figure $g$ is given in Table 2. Points were plotted with respect to the blackbody curve. The Drightness of the blank was several magnitudes less than the calibration blackbody and the points fitted reasonably well on a blackbody shaped curve, showing that the aluminum thin film on glass emitted as a graybody with low emissivity. The brightness of the 


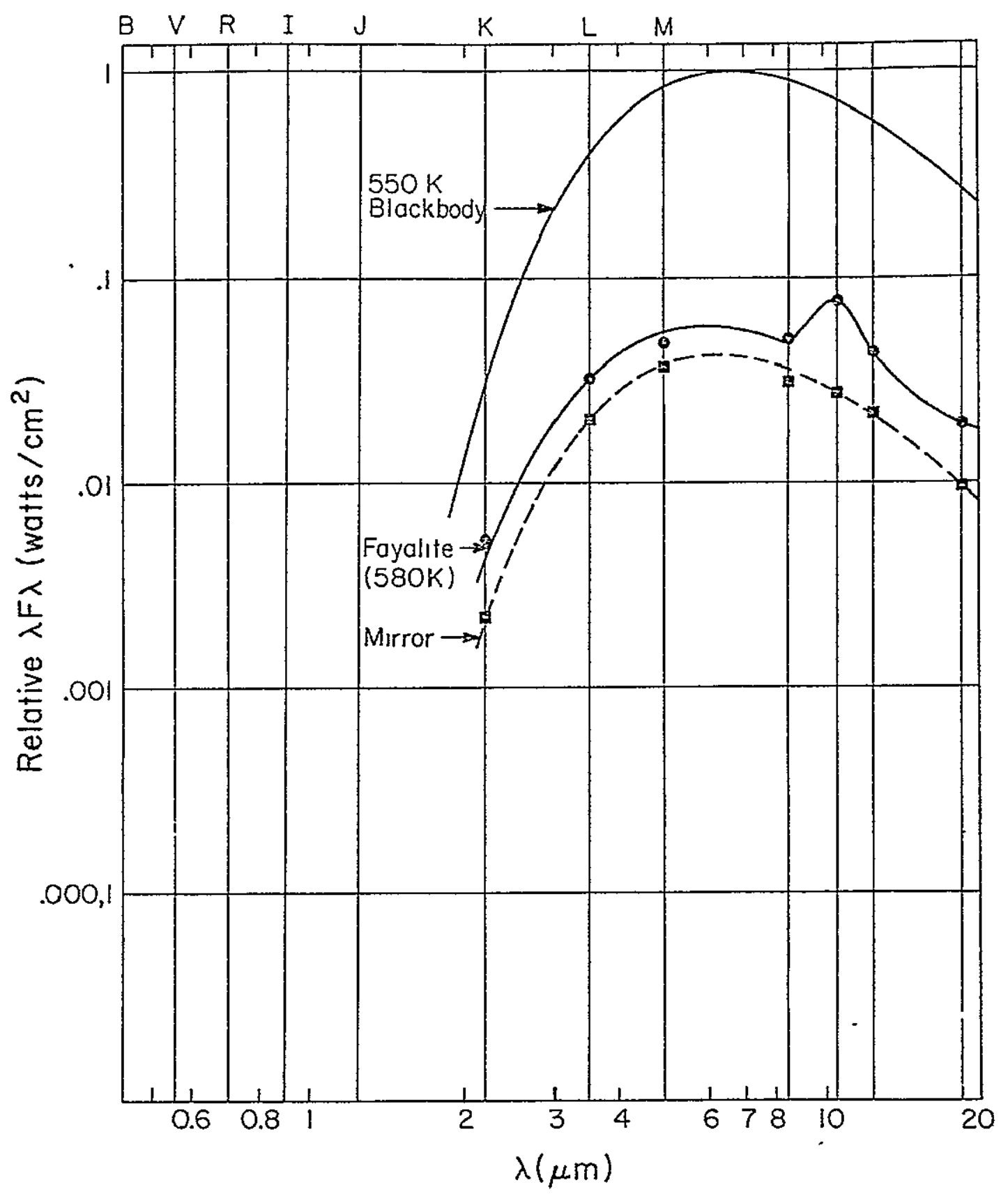

Fig. 9. Laboratory broadband emission spectrun of fayalite. 
TABLE 2

DETECTOR SIGNAL VOLTAGES AND CALCULATED MAGNITUDES OF A FAYALITE SAMPLE, USING BROADBAND PHOTOMETRY

$\begin{array}{ll}\lambda(\mu \mathrm{m}) & \begin{array}{l}\text { Laboratory } \\ \text { Blackbody } \\ 550 \mathrm{~K} \\ \text { (m volts) }\end{array} \\ 2.2 & .0 .515 \\ 3.5 & 6.33 \\ 4.8 & 7.4 \\ 8.5 & 3.25 \\ 10.6 & 2.4 \\ 12.5 & 1.84 \\ 18 & 1.04\end{array}$

\begin{tabular}{cc}
$\begin{array}{c}\text { Fayalite } \\
(0-.5 \mu \mathrm{m}) \\
580 \mathrm{~K} \\
(\mathrm{~m} \text { volts })\end{array}$ & $\frac{\Delta \mathrm{m}}{.081}$ \\
\hline 0.51 & 2.01 \\
.41 & 2.73 \\
.179 & 3.14 \\
.26 & 3.15 \\
.14 & 2.41 \\
.076 & 2.80 \\
& 2.84
\end{tabular}

\begin{tabular}{|c|c|}
\hline $\begin{array}{l}\text { Mirror } \\
580 \mathrm{~K} \\
\text { (m-volts) }\end{array}$ & $\Delta \mathrm{m}$ \\
\hline .0362 & 2.88 \\
\hline .325 & 3.22 \\
\hline .31 & 3.44 \\
\hline .112 & 3.66 \\
\hline .092 & 3.54 \\
\hline .071 & 3.54 \\
\hline .037 & 3.62 \\
\hline
\end{tabular}


fayalite sample at short wavelengths is also several magnitudes less than the calibration blackbody but is somewhat greater than the blank substrate.

From tabulated data for fayalite, a rough estimate of the mass absorption coefficient can be calculated. The optical depth, $\tau$, of the sample is equal to the ratio of the difference between the grain sample signal and the mirror blank signal to the blackbody signal.

In equation form

$$
\begin{aligned}
\tau & =\frac{V g-V m}{V b} \\
& =(x)(\rho)(d)(f)
\end{aligned}
$$

$$
\text { where } \begin{aligned}
V g= & \text { grain sample signal } \\
V m= & \text { mirnor blank signal } \\
V B= & \text { blackbody signal } \\
X= & \text { mass absorption coefficient in } \mathrm{cm}^{2} / \mathrm{gm} \\
\rho= & \text { density in gm }(\mathrm{cm})^{3} \\
a= & \text { grain diameter }(\mathrm{cm}) \\
f= & \text { fraction of mirror surface covered } \\
& \text { by grains. }
\end{aligned}
$$

The mass density of fayalite is $\sim^{4} \mathrm{gm} /(\mathrm{cm})^{3}$. Most of the grains were in the range from .25 to $.5 \mu \mathrm{m}$ in size and $20 \%$ of the mirror surface was covered by 
grains as determined from an electron micrograph. Using voltage signals at $10.6 \mu \mathrm{m}$, which is near the center of the silicate absorption band of fayalite, the value of $x$ is

$$
\begin{aligned}
x & =\frac{(V g-V m) / v b}{\rho d f} \\
& \simeq \frac{(.26-.09) / 2.4}{(4)\left(.3 \times 10^{-4}(.2)\right.} \\
& \simeq 3000 \mathrm{~cm}^{2} / \mathrm{gm} .
\end{aligned}
$$

This value is perhaps a factor of 2 too low but the error is attributed to uncertainties in the actual grain size distribution and in the fraction of the minror covered by gnains.

In Fìgure 10, several curyes are displayed for the purpose of making comparisons. Absolute values of $\lambda F_{\lambda}$ are not indicated, Dut relative values of $\lambda F_{\lambda}$ between a sample cunve and its accompanying blank substrate curve can be determined.

The upper two curyes in Figure la show the. infrared spectra of comets Bennett and Kohoutek CMaas et al. 197a; Ney 1974bI. Thein spectra exhibit a thenmal continuum with an emission featune superimposed at lajm. The next curve is the laboratory spectrum of a fine-grained dunite sample. This material was chosén because it is thought that enstatite $\mathrm{CMg} \mathrm{SiO}_{3} \mathrm{I}$ and forsterite $\mathrm{CMg}_{2} \mathrm{SiO}_{4} \mathrm{l}$ would 
be among the first materials to condense in a stellar atmosphere. The dashed curve represents an aluninum thin film on glass substrate. The dunite feature at $10 \mu \mathrm{m}$ resembled very strongly the feature seen in. Comets Bradfield and Kohoutek. An excess was also seen at $20 \mu \mathrm{m}$, in agreement with the cometary spectra.

Several materials were investigated using the broadband photometer and the results are summarized in Figure 10. For these curves the particles were, for the most pant, less than .5um in size. The olivines (dunite and fayalite)and Allende (Type 3 carbonaceous chondritel gave the best fit to the cometary spectra, although anorthite and Murcay also showed a lajm feature. Anorthite was chosen for the study because of its similarity in chemical composition to much of the lunar soil (Wood 1975 ). As has been mentioned, minerals similar in composition to anorthite are thought to be among the first silicates to condense in a cooling nebula of solar composition (Grossman and Larimen 197't). The broadband measurements showed that quantz can be ruled out as a majon component of cometany grains (Gammon et al. I9722.

The 2-8um thermal continuum partions of the laboratory curves in Figure IU were due to emission 


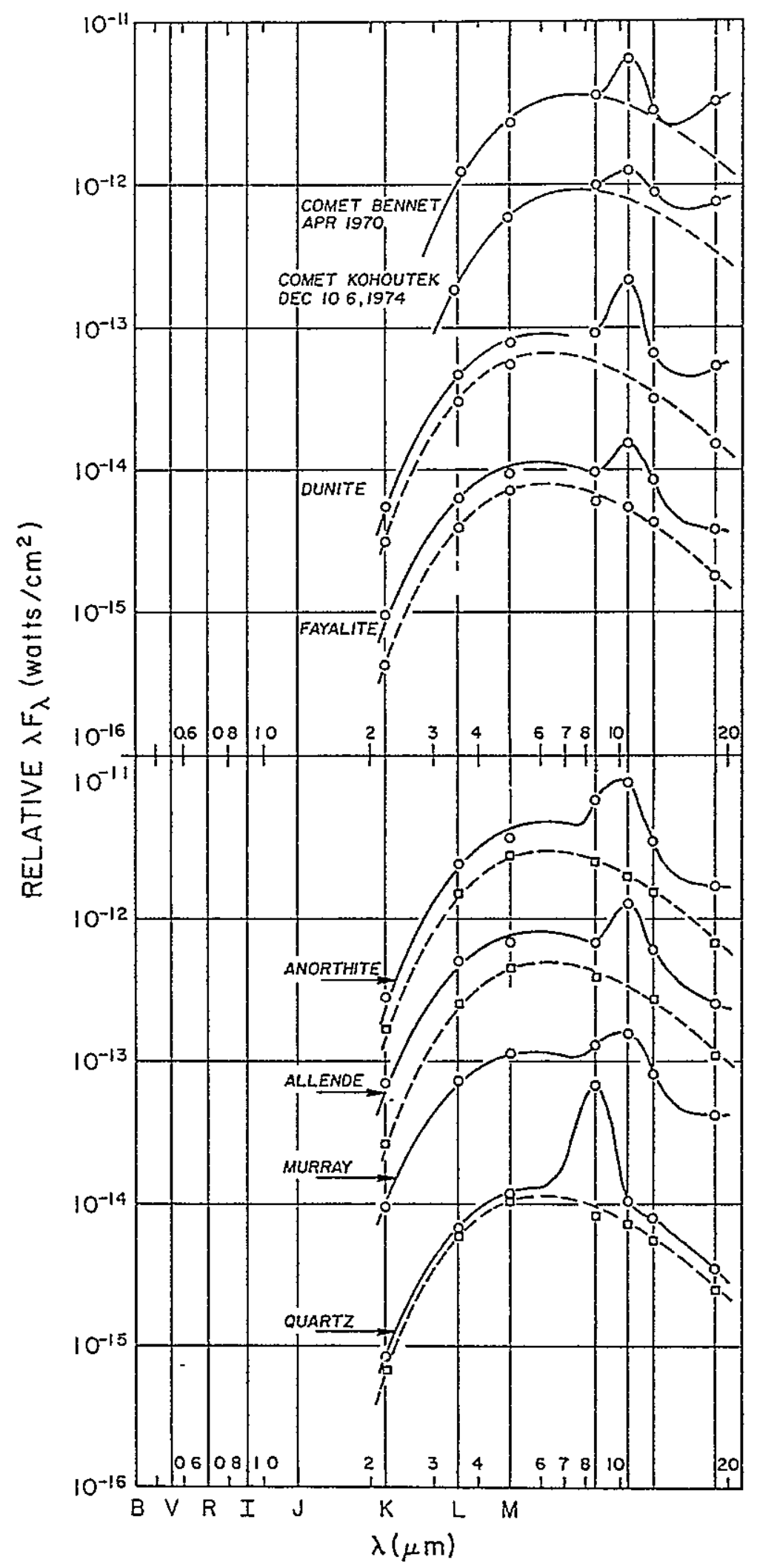

Fig. 10. Broadband spectra of Comets Bennett and Kohoutek (Maas et al.1970; Ney 1974b). Laboratory broadband emission spectra of terrestrial silicates and meteorites. 
from the substrates and emission from the grains. The quartz curve was closest to the blank substrate curve while the Allende curve lay significantly above the curve of the mirron. The terrestrial silicates dunite, fayalite and anorthite were intermediate between quartz and Allende in the amounts with which they exceeded the brightness of the blank substrates. Although these effects depend somewhat on the amount of grain material on the substrate, it can be seen that a "clean" crystalline silicate such as quartz has very little opacity on the short wavelength side of the Si-O vibrational absorption band but that Allende, a Type 3 carbonaceous chondrite, has an appreciable opacity outside the absorption band.

A plot should he made of the difference between the sample and blank substrate curves. However, in the $2-8 \mu \mathrm{m}$ region this involved plotting the difierence of two values of $\lambda F_{\lambda}$ which were fairly close together resulting in considerable scatter in the data and points that did not fall too well on a blackbody curve. CThis is in contrast to the cometary data in this range which fitted a blackbody curve quite we11.) This subtraction was not done and therefore the curves in Figure 10 simulate the cometary spectra prisnarily in the region of ifre 10jm feature. It 
should be emphasized, however, that the silicate samples, with the exception of quartz, have an appreciable opacity in the $2-8 \mathrm{jm}$ range that is greatest for the carbonaceous chondrites which contain carbon and hydrocarbons (Wood 1963). This agrees with the results by Mazs et al. (1970) who postulated a black component in cometary grains to account for the blackbody portion of the Comet Bennett infrared spectrum.

The low resolution of the broadband photometer made the selection of a best material from among the ones shown in Figure 10 very difficult. Comparisons using intermediate resolution spectrophotometry were more discriminating.

For all of the materials listed in Figure 10, runs were made for a number of temperatures ranging from $\sim 350 \mathrm{~K}$ to $\sim 800 \mathrm{~K}$ in an attempt to simulate the change of temperature that comets experience as they pass near the sun. The resulting family of curves of Allende are shown in Figure 11. For each curve the spectrum is that of a thermal continuum with a silicate feature superimposed. Similar spectra of the other materials of Figure 10 are not shown because no change in the shape of the silicate features due to change in sample temperature was seen. 


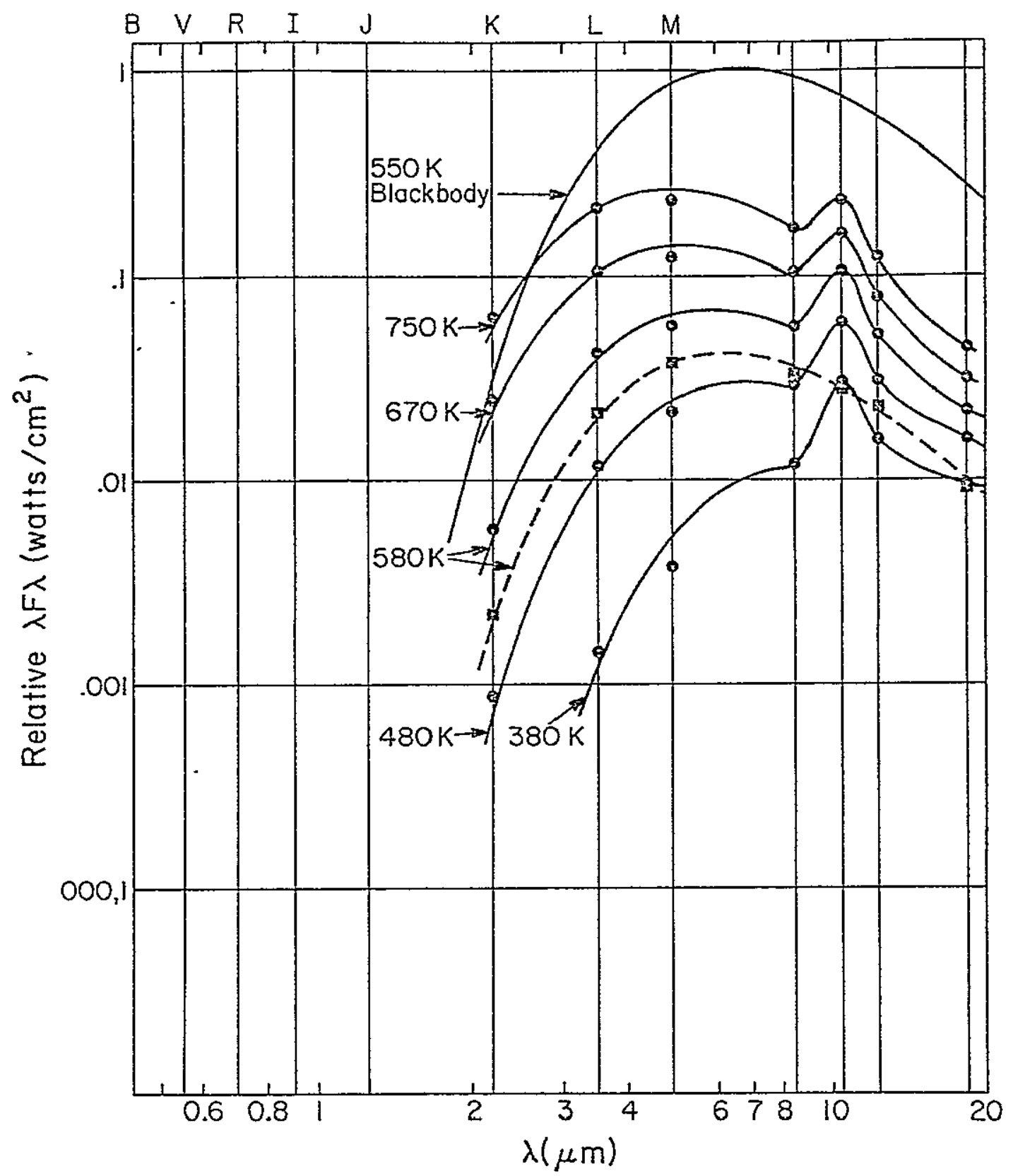

Fig. 11. Laboratory broadband emission spectra of Allende ( $0-.5$ m size range). 
The effect of grain size on the 10 m dunite feature is shown in Figure 12. The numbers on the graph indicate the range of grain sizes in $\mu \mathrm{m}$ for each sample. For samples with grains larger than $37 \mu \mathrm{m}$, no feature was seen but as the grain sizes decreased a feature began to appear. A very weak excess was noticed first for grains in the 25 to 37 m range; for smaller sized particles, the feature was very much in evidence. This figure summarizes the results of investigating 7 different samples with the only difference being in the sizes of the grains. A temperature of approximately $500^{\circ} \mathrm{K}$ was used for these runs. The curves were placed on the graph in order of decreasing grain size. The fact that one curve is above another does not mean that it necessarily was brighter in emission. The results of this part of the study are in qualitative agreement with the discovery by Ney (1974a) that the particles in the anti-tail have no signature (and therefore should be largel and the prediction by Sekanina (1974) that the particles which make up the anti-tail are large.

\section{SPECTROPHOTOMETRY}

Absorption spectra of fine grain forsterite show an'irregular feature with two peaks, one near 


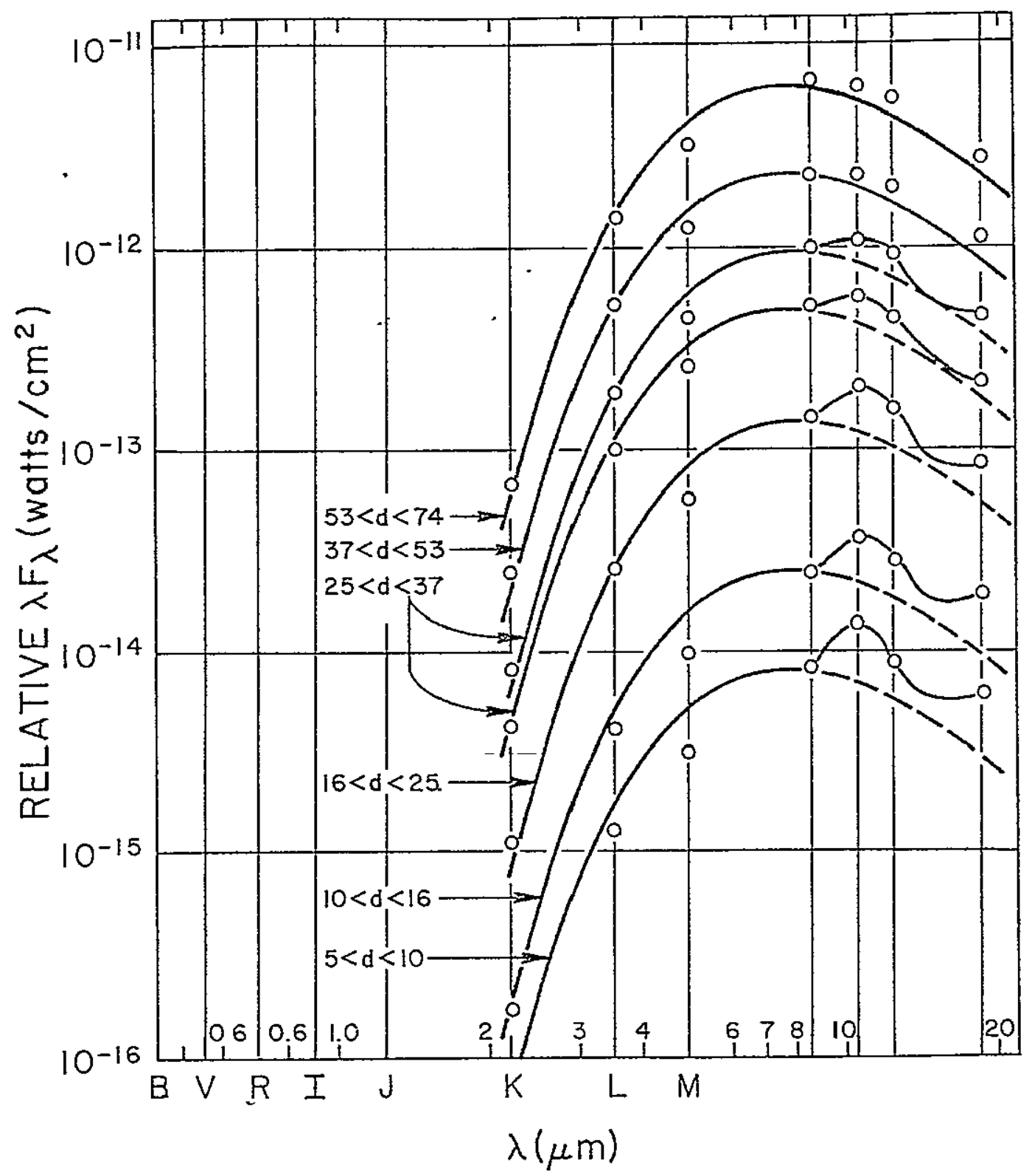

Fig.12. Laboratory spectra of dunite samples with different grain sizes. 
$10 \mu \mathrm{m}$ and the other near $11 \mu \mathrm{m}$ (Zaikowski et al. 1975a; Day 1975). These spectra do not agree at all with the intermediate resolution absorption spectra of such objects as the galactic center which show a smoothly varying absorption curve with a single peak near 9.7um (Woolf 1973a). Figure 14 shows a plot of the infrared emission of Comet Kohoutek taken by Merrill (1974). It also shows a smoothly varying curve with a single peak near $9.8 \mu \mathrm{m}$. These data suggest that although olivine gives a good fit to the cometary broadband spectra, it would not match the intermediate resolution spectra. Therefore, it was decided to obtain the intermediate resolution emission spectra of the materials which had been studied with the broadband photometer to see if any of them would resemble the emission spectrum of Comet Kohoutek. Additional materials were added to this part of the study.

The experimental setup was the same as for the broadband measurements except that the multi-filter dewar was replaced by a dewar containing a spectrometer filter wheel. The filter wheel was in two segments. One segment spanned the wavelength range from $7.5 \mu \mathrm{m}$ to $14 \mu \mathrm{m}$ and. the other went from $3 \mu \mathrm{m}$ to $5 \mu \mathrm{m}$. This filter wheel was identical to the one used 
by Merrill for the Comet Kohoutek measurements; its resolution for the long wavelength segments was such that $\Delta \lambda / \lambda=.01$. The spectrometer filter wheel turned continuously. As many points as were necessary were taken from the chart recorder output to plot an emission feature. Because the silicates have no spectral features in the $3-5.5 \mu \mathrm{m}$ range, the emphasis in this study was placed on the 7.5-

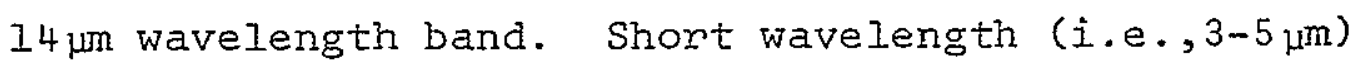
data were taken for all samples; however, a spectrum of only one sample is shown (Figure 13). Because accurate mass deteminations were not made of the samples, calculations of mass absorption coefficients were not made. As in the broadband case, the brightness of a blank substrate or grain sample was calculated in magnitudes with respect to a - calibration blackbody and the points were plotted with respect to a blackbody curve. Figure 13 shows a plot of $\lambda B_{\lambda}$ where $B_{\lambda}$ is the Planck function in watts $/ \mathrm{cm}^{2} \mu \mathrm{m}$. The plot has been normalized to $I$ at $\lambda=6.67 \mathrm{~m}$, the wavelength position of the peak value of $\lambda \mathrm{B}_{\lambda}$ for $\mathrm{T}=550^{\circ} \mathrm{K}$. As mentioned earlier, $550^{\circ} \mathrm{K}$ was the temperature of the laboratory blackbody source. The lower curves are spectra of the dunite sample and a blank substrate. To get a 


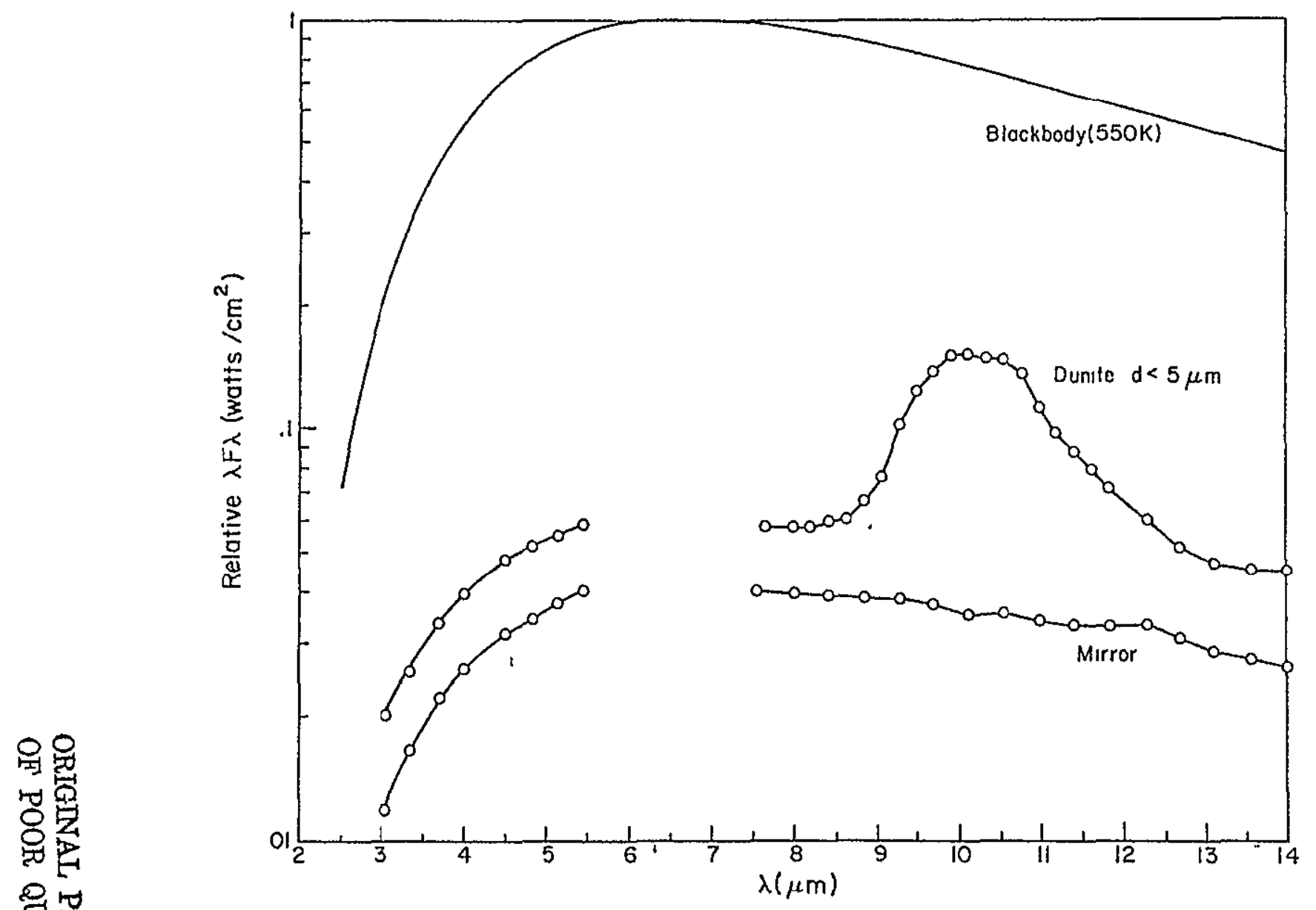

Fig. 13. Laboratory intermediate resolution emission spectrum of dunite. 
curve which represents the emission of the grains, the $\lambda F_{\lambda}$ curve of the blank substrate was subtracted from the $\lambda F_{\lambda}$ curve of the grain sample.* It is this emission curve which was then compared with the Comet Kohoutek spectrum, because the comet Kohoutek spectrum shown by Merrill was obtained by subtracting a thermal background from the initial spectrum.

Figure 14 gives a summany of the results for the olivines and other iron-magnesium silicates, with the Comet Kohoutek spectrum shown at the top. The $0-.5 \mu \mathrm{m}$ dunite and fayalite samples have features which are narrow compared to the Comet Kohoutek spectrum and their peaks are seen to be at wavelengths which are too long to match the cometary feature. The effect of langer grains (and possibly more material on the substratel is shown for the

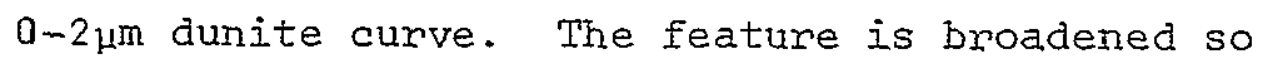
that it resembles more closely comet Kohoutek on the long wavelength side, but the peak at $10.3 \mu \mathrm{m}$ and the slope on the short wavelength side of the feature still are not a good fit to the cometary spectrum. The next curve shows the emission spectrum of the olivine slag sample. The peaks near $10.3 \mu \mathrm{m}$ and * Appendix A gives additional details concerning data reduction for this part of the study. 
11.6 $\mathrm{m}$ are characteristic of olivine. The slag spectrum is not a good fit to the cometary feature. An amorphous magnesium silicate was prepared by combining sodium meta-silicate and magnesium chloride in the correct proportions in water. The amorphous silicate precipitates as a gel (Day 1974). The following reaction took place:

$$
\mathrm{Na}_{2} \mathrm{SiO}_{3}+\mathrm{MgCl}_{2} \rightarrow \mathrm{MgO}+\mathrm{SiO}_{2}+2 \mathrm{NaCl} \text {. }
$$

The material was washed several times in water to remove the $\mathrm{NaCl}$, oven dried at $125^{\circ} \mathrm{C}$ for approximately 4 hours, and ground in an agate mortar and pestle to produce fine grains. The grains were sized and deposited on substrates using sedimentation techniques outlined in the section on sample preparation. The emission spectra of this material show peaks near 9.8 $4 \mathrm{~m}$ which are correct to match cometary and astrophysical maxima. The broader feature of the $2 \mu \mathrm{m}$ sample also matches the cometary feature fairly well on the short wavelength side. These results suggest (in agreement with Day's conclusions 2 that such a material could be an important component of cometary and interstellar grains. There is a slight shift of the peak emission toward longer wavelengths for the sample with larger grains, an effect also seen in other materials in 
this study. The spectrum of an $0-2 \mu \mathrm{m}$ amorphous magnesium silicate material which had been previously heated to $1200^{\circ} \mathrm{C}$ is seen to show structure and to be broader than the spectrum of its "uncooked" counterpart. The peaks at $9.4 \mu \mathrm{m}, 10.8 \mu \mathrm{m}$ and $11.8 \mu \mathrm{m}$ agree fairly well in position to the absorption peaks of enstatite reported by Zaikowski (1975a). Heating the amorphous silicate material alters its structure to that of crystalline enstatite. This agrees with the results obtained by Day (1974) when working with a proto-forsterite material. Day also showed that no $x$-ray diffraction lines were seen for the low temperature gel sample but that the full run of forsterite lines were present for a sample that had been heated to $750^{\circ} \mathrm{C}$. The appearance of features in the heated amorphous silicate suggests that if this material is a component of cometary grains it must exist in an unheared form.

The bottom curve in Figure 14 shows the emission spectrum of Abee, an enstatite chondrite meteorite. The strong peak near $9.3 \mu \mathrm{m}$ and the weaker peak near $10.6 \mu \mathrm{m}$ resemble the spectrum of the synthetic enstatite material just above it. The Abee spectrum has a slope in the $8-9 \mu m$ region that matches Comet 


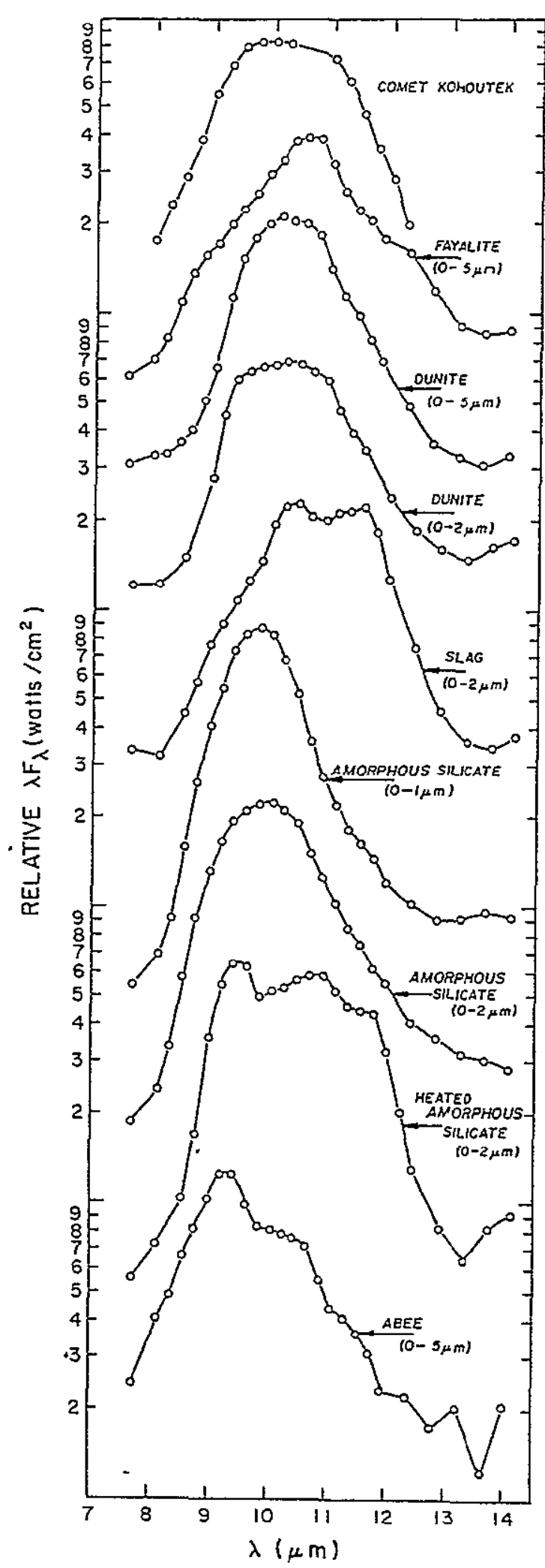

81

Fig. 14. Spectrum of Comet Kohoutek (Merrili 1974). Laboratory emission spectra" of olivines, amorphous silicates and an enstatite chondrite. 
Kohoutek closely; other than that, it is not a good fit.

Emission spectra of the clay mineral montmorillonite and of several carbonaceous chondrite meteorites are shown in Figure 15. The Comet Kohoutek spectrum is shown again to facilitate comparisons. The strong similarity between the spectrum of fine grained $(d<.5 \mu \mathrm{m})$ montmorilionite and the spectra of Orgueil and Ivuna (both $\mathrm{CI}$ materials) is in agreement with the conclusion that hydrous layer lattice silicates are a major phase of Cl meteorites (Bass 1971). The spectra of these three materials show peaks in the range from 9.6 to $9.8 \mu \mathrm{m}$, but the features are quite narrow compared to the cometary feature.

A 0-2um sample of montmorillonite yields a spectrum which is Droadened but which retains the peak near $9.6 \mu \mathrm{m}$. However, the broadened feature is not a good fit to the cometary feature.

Two additional runs of the $0-.5 \mu \mathrm{m}$ montmorilionite sample were made at $380^{\circ} \mathrm{K}$ and $750^{\circ} \mathrm{K}$ nespectively. The spectra are not shown but they were similar in shape to the $580^{\circ} \mathrm{K}$ spectrum shown in Figure 15 and the position of the peak did not change. 
Zaikowski et al. (1975a)have concluded that hydrous silicates are an important component of interstellar grains and cometary grains because they possess a peak near $9.8 \mu \mathrm{m}$ and are an important phase of the primordial Type 1 carbonaceous chondrites. The findings of this study are not inconsistent with their conclusions. However, the narrowness of the emission spectra of montmorillonite, Orgueil and Ivuna would require the presence of other materials in the cometary grains to broaden the feature. High temperature condensates such as forsterite, enstatite or anonthite are possibilities.

The Murray meteorice has hydrous layer lattice silicates and high temperature condensates (e.g. olivine and pyroxene) in approximately equal proportions (Zaikowski et al. 1975a). The emission spectrum for the $0-.5 \mu \mathrm{m}$ grain size sample is similar to the cometary features; however, it exhibits some structure in the $9 \mu \mathrm{m}$ to $11 \mu \mathrm{m}$ region and is slightly broader. The spectrum of the $0-2 \mu \mathrm{m}$ sample is quite similar to that of the fine grained Murray sample, showing some structure near $10.1 \mu \mathrm{m}$ that is possibly due to the olivine component. The Allende meteonite, a C3 material, yields a spectrum in the $10 \mu \mathrm{m}$ to $12 \mu \mathrm{m}$ region which is a 


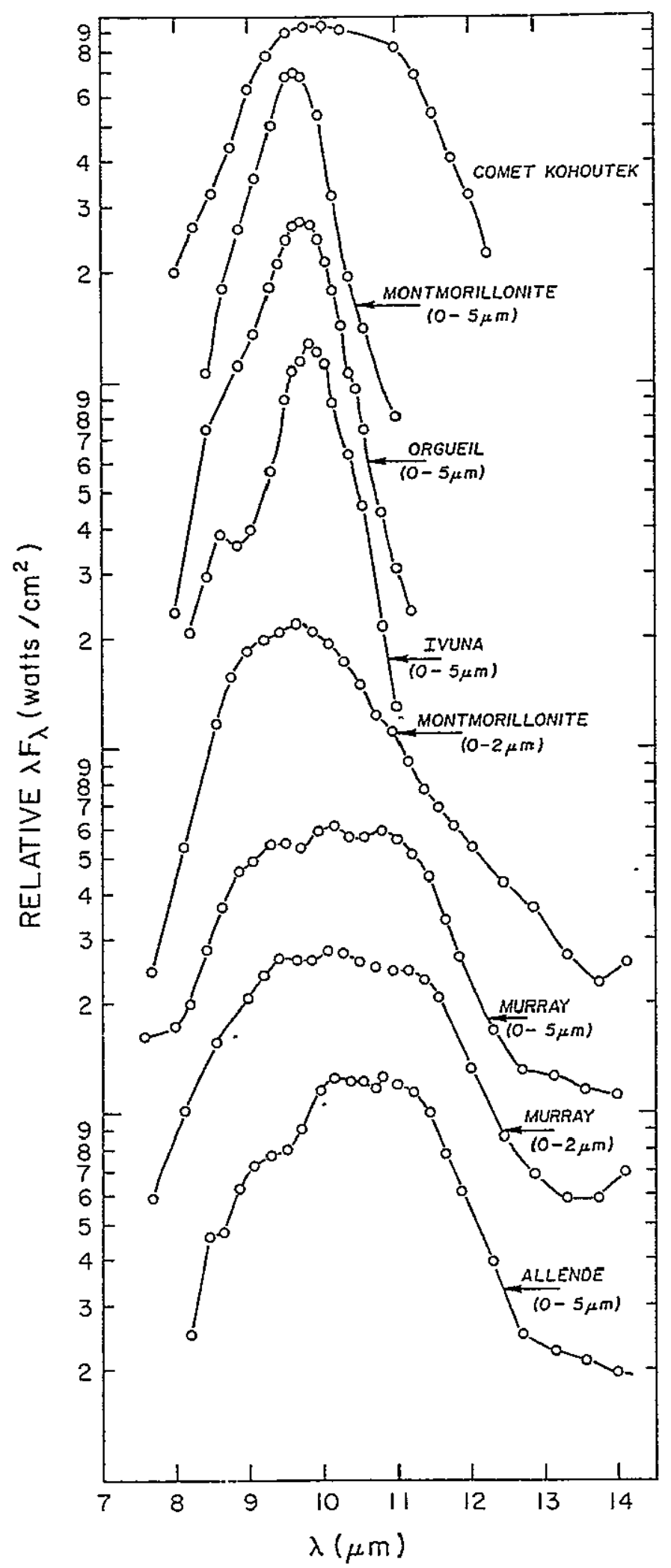

84 
reasonably good fit to the cometary feature. The absence of hydrous silicates in its composition causes it to depart greatly from the cometary feature in the 9-10 $\mu \mathrm{m}$ wavelength region.

Figure 16 shows emission spectra of anorthite. The $0-.5 \mu \mathrm{m}$ sample gives a good fit to the cometary spectrum with regard to width and the slopes in the $8-9 \mu \mathrm{m}$ and $11-12 \mu \mathrm{m}$ regions, but the strong dip at $9.8 \mu \mathrm{m}$ differs considerably from the cometary feature.* The spectrum for the 0-Ium grain size anorthite sample resembles the $0-.5 \mu \mathrm{m}$ grain size sample with regard to the position of its double peaks. Its fit to the cometary spectra is less satisfactory. The next curve shows the effect of irradiation on anorthite using $10 \mathrm{keV}$ protons. The beam current was such that irradiation for I0 hours gave a charge dose of $\sim 6$ coul $/(\mathrm{cm})^{2}$. The charge dose was chosen to be roughly equal to dosages used by Gold (1976) who found that proton irradiation of pulverized Iunar rock samples caused the rock material to develop a surface chemistry (i.e., the iron/oxygen ratio) and albedo similar to those of Iunar soil. In Gold's experiments $2 \mathrm{keV}$ *In preparing the $0-.5 \mu \mathrm{m}$ anorthite sample some of the quartz impurity was removed through the use of an intermediate density liquid flotation technique. This separation was not done for the a-1um anorthite sample. 
protons and charge doses of approximately 3 coulombs $/ \mathrm{cm}^{2}$ were used. The proton bombardment of the anorthite caused a smoothing of the feature in the 9-IIum region, a charge of the slope in the Il to $12 \mu \mathrm{m}$ region, and an increase in the opacity of the sample in the 12 to $14 \mu \mathrm{m}$ region. The spectrum of the irradiated sample gives a better fit to the cometary feature in the $10-12 \mu \mathrm{m}$ region than does the unirradiated sample, although the match is not particularly good in the $8-10 \mu \mathrm{m}$ region. The irradiation experiment demonstrates that structure in the silicate feature can be smoothed by bombardment using low energy protons. It is assumed that the proton bombardment destroys the periodicity in the silicate, washing out the structure in the emission feature.

Albite, the sodic end member of the plagioclase feldspar series, also exhibits a twin peaked emission spectrum which does not match the cometary spectrum at all. Its peaks are shifted toward short wavelengths by $\sim .5 \mu \mathrm{m}$ when compared to the anorthite emission spectra.

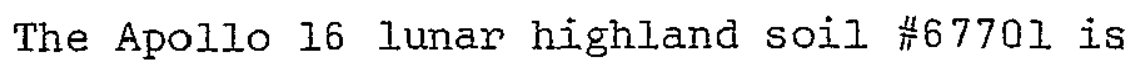
largely anorthositic, but it has a weathering history which is quite different from that of 


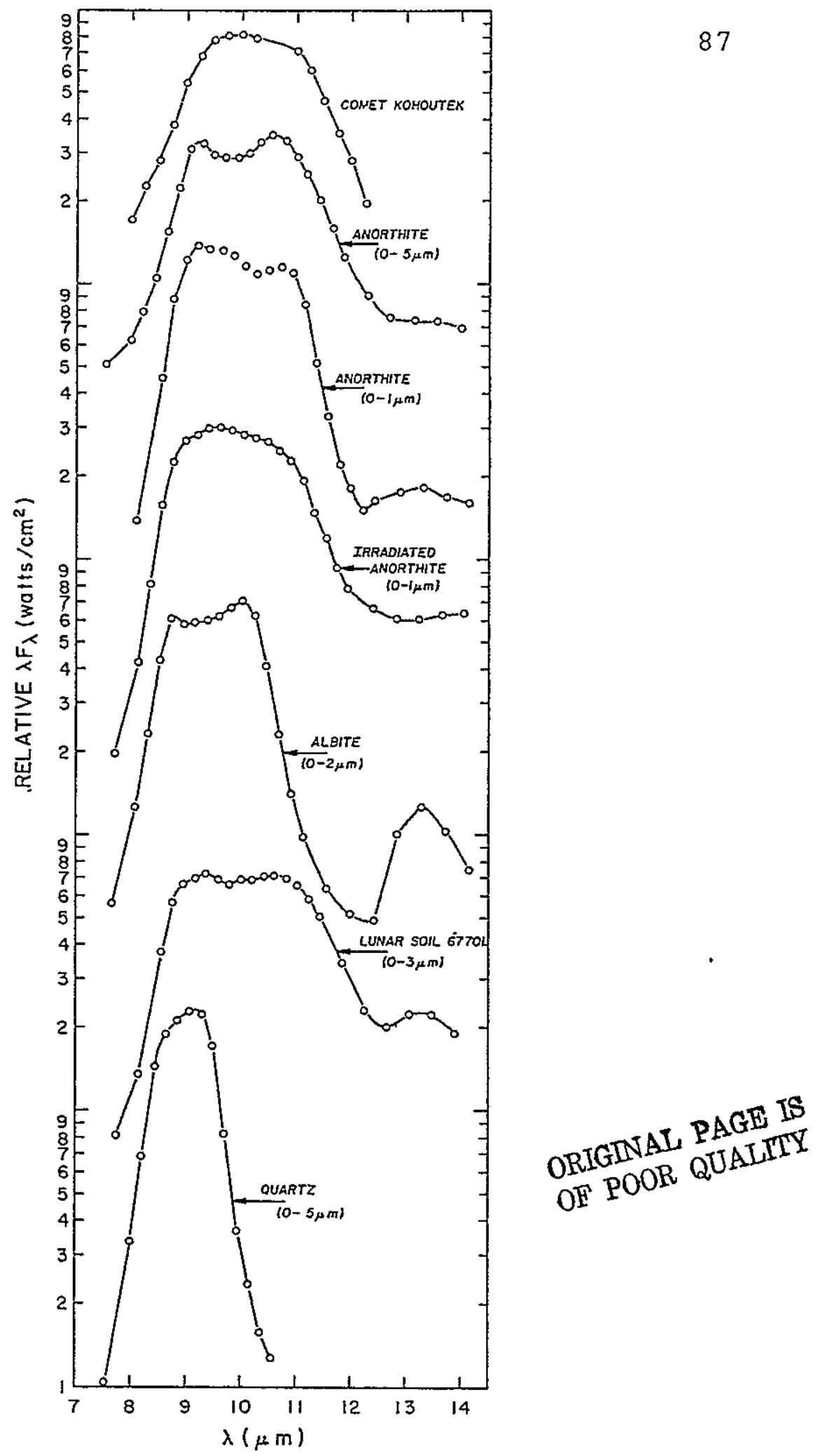

Fig. 16. Laboratory emission spectra of plagioclase feldspars, a lunar soil and quartz. 
terrestrial minerals. Its spectrum resembles the spectra of terrestrial anorthite shom in this figure. The shape of its emission feature also resembles the cometary feature with the exception of the strong dip at $9.8 \mu \mathrm{m}$ and a somewhat steeper slope on the short wavelength side.

The final spectrum in Figure 16 is of quartz. Because of its peak near $9.2 \mu \mathrm{m}$, it can be ruled out as an important component of cometary grains. The large displacement of the quantz peak from the cometary peak was detectable in the broadband results. Cammon et al. (1972) have pointed out that emission from quartz is at too short a wavelength to match circumstellar spectra.

Figure 17 shows spectra of two Apollo 15 lunar mare basalt surface soil. samples. The $0-3 \mu \mathrm{m}$ sample is overall an excellent fit to the cometary spectrum except for a slight dip at $9.8 \mu \mathrm{m}$. The spectrum of a second mare basalt 15531 sample, using grains near $4 \mu \mathrm{m}$ in size, is somewhat broader with a higher opacity at long wavelengths. Grinding of lunar samples was not done because such treatment would have altered the amorphous surfaces which lunar grains acquire due to weathering by solar wind irradiation and micrometeorite bombardment. Since 
grinding was not performed on the lunar materials, the grain sizes are somewhat larger than those used for other samples simply because submicron portions of unground lunar soil were not available. In the case of the $0-3 \mu \mathrm{m}$ samples, this was not a serious difficulty. It is thought the $0-3 \mu \mathrm{m}$ spectra give a good indication of the emission properties of fine grain lunar materials.

Juvinas, a basaltic achondrite, is a member of a compositional group called eucrites in which lathlike structures of plagioclase are enclosed in a matrix of clinopyroxene. The basaltic achondrites are analogous to Iunar basalts (Duke and Silver 1967). The emission feature of the $0-1 \mu \mathrm{m}$ Juvinas sample has a sharp spike at $9.4 \mu \mathrm{m}$ which does not appear in the spectrum of the $0-2 \mu \mathrm{m}$ grain size sample. This could be a grain size effect. The $0-2 \mu \mathrm{m}$ sample displays a broader feature and its overall fit to the cometary spectrum is quite good except for a dip in the $10 \mu \mathrm{m}$ region. Its spectrum also matches closely the 15531 lunar soil energy spectrum of $0-3$ um grains. Diabases are a class of terrestrial igneous rocks analogous in texture and mineralogy to eucrites. They are associated with magma injected into cracks in the earth's crust resulting in a cooling rate 


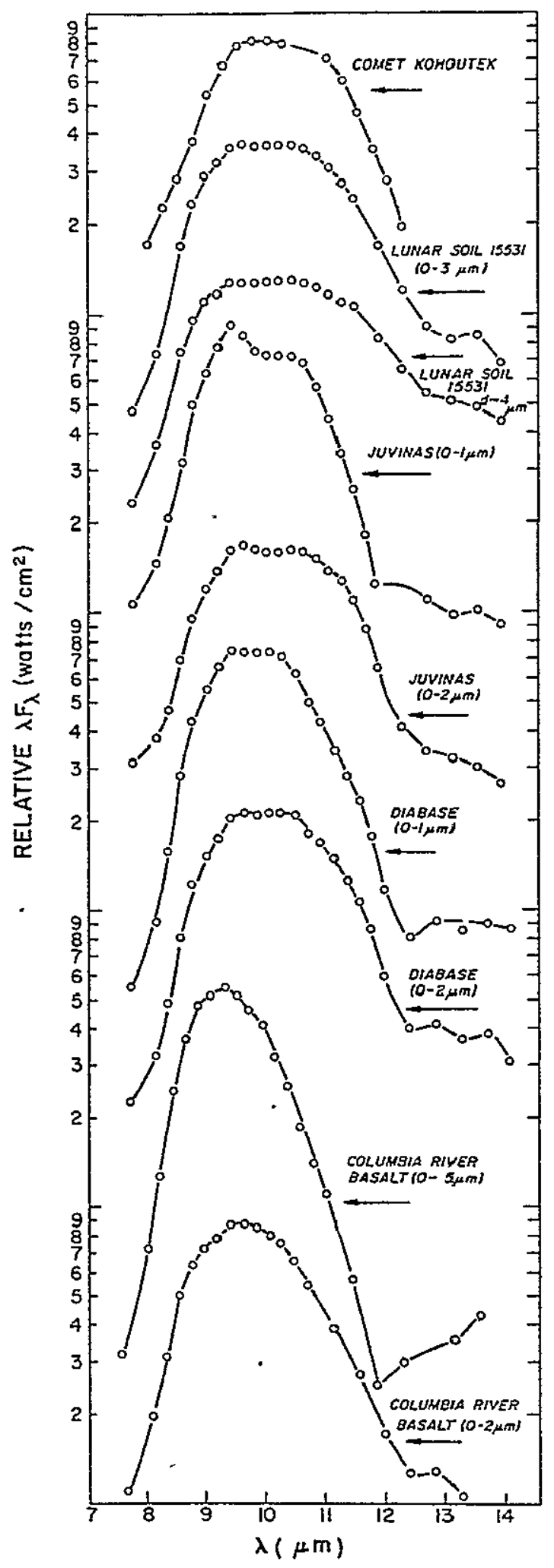

Fig, 17. Laboratory emission spectra of a lunar mare soil, a basaltic achondrite, a terrestrial diabase, and a terrestrial basalt. 
intermediate between the rapid crystallization of magma poured onto the surface of the earth from volcanoes and the very slow cooling of magma trapped beneath the earth's surface (Wood 1963). The next two curves in Figure 17 are emission spectra of a sample of a Lake Superior diabase. There is general agreement between these emission curves and the cometary spectra, although the 0-I!!m diabase spectrum does not fit the cometary spectrum as well as does the $0-2 \mu \mathrm{m}$ Juvinas spectrum.

The final curves in Figure 17 show spectra of Columbia River basalt samples. Their features do not match the cometary curve in the 10-12um range; however, the fits are somewhat better on the short wavelength side. Compared to the diabase sample, Columbia River basalt has more normative quartz and plagioclase but less pyroxene.

Figures 18 and 20 show absorption spectra of certain samples which are thought to be importan as possible components of cometary and interstellar dust grains. Emission spectra of these same samples have been reproduced from other figures in this paper for comparison purposes and are shown in Figures 19 and 21 . 
Figure 18 shows the absorption spectrum of the olivine slag material. The twin peaks in its spectrum at 10.3 and $11.5 \mu \mathrm{m}$ agree with published data for olivine (Zaikowski 1975a). Also the wavelengths at which these peaks occur match fairly well the slag emission spectrum peaks. Neither the emission nor the absorption spectra of this material match the smoothly varying astrophysical features so that crystalline olivine cannot be the major constituent of the astrophysical grains.

The synthetic magnesium silicate and montmonillonite show smoothly varying features that peak near $9.8 \mu \mathrm{m}$, and in this respect are similar to astrophysical absorption features.

The absorption spectrum of the heared synthetic silicate is identical to. that of enstatite; its fit to an astrophysical absorption feature is poor. Comparison of the synthetic silicate absorption feature taken at room temperature to the $550^{\circ} \mathrm{K}$ emission spectrum shows that structure near $9.9 \mu \mathrm{m}$ and $11.2 \mu \mathrm{m} \cdot \mathrm{has}$ been muted or smoothed out in the emission feature. The smoothing is attributed to the interaction between vibrational modes of the lattice and its incoherent thermal agitation (Simon and McMahon 1953). 


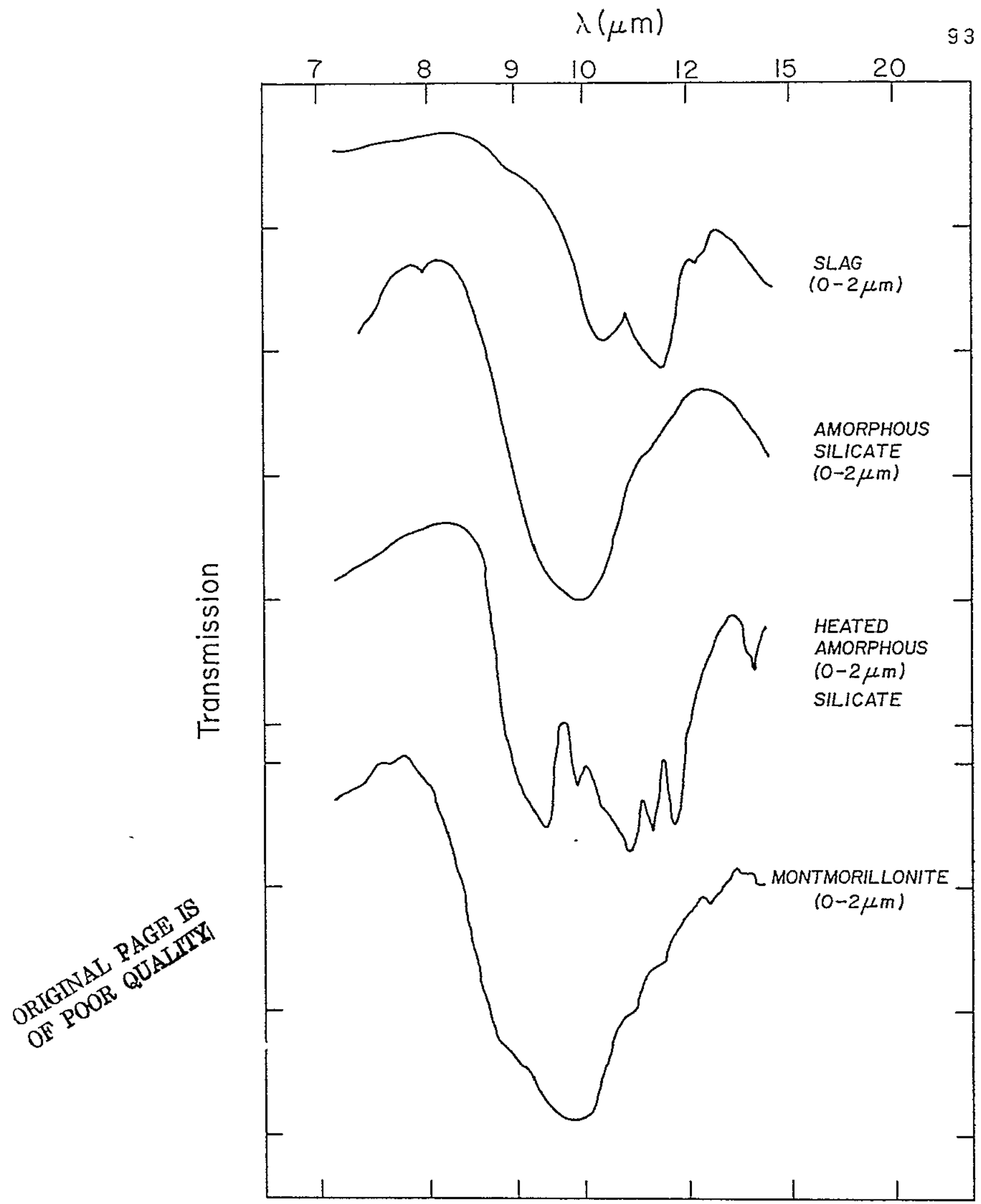

Fig. 18. Laboratory absorption spectra of olivine slag, amorphous silicates and montmorillonite. 


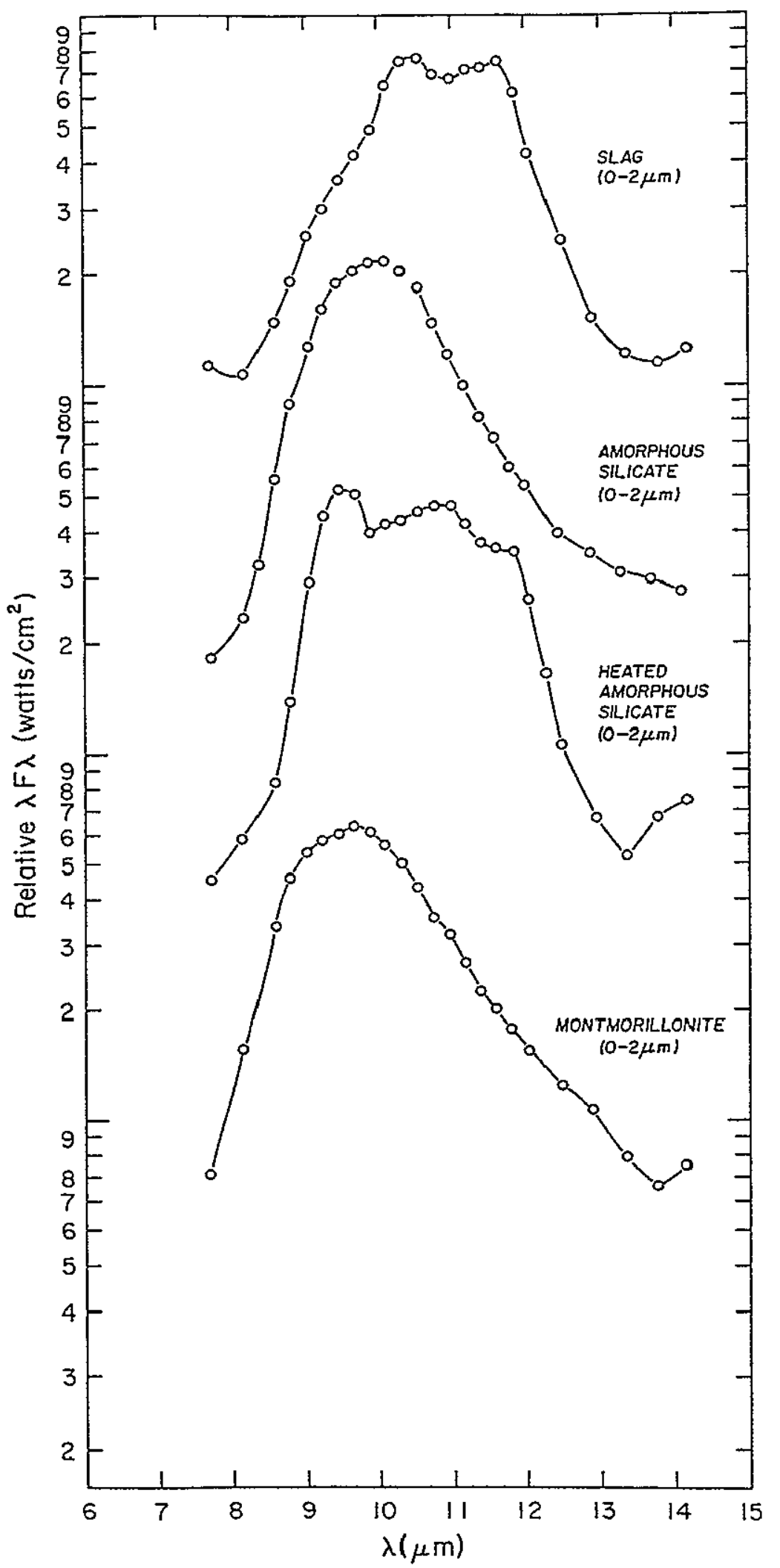

94 
Figure 20 shows the absorption spectrum of a 0-Ium anorthite sample and Figure 21 shows its corresponding emission spectrum. A weak feature near $9.6 \mu \mathrm{m}$ appears in absorption but is muted in emission. The absorption spectrum of anorthite does not match astrophysical absorption features. The absorption spectrum of Juvinas shows peaks near $9.5 \mu \mathrm{m}$ and $10.6 \mu \mathrm{m}$. The positions of these peaks agree closely with those reported by Gillett and Soifer (1976) in the infrared absorption spectrum of an extended infrared source at the positions of the $\mathrm{OH}$ maser source $\mathrm{OH} 231.8+4.2 \equiv$ OH 0739-14. However, a thind peak in the Juvinas spectrum at $11.3 \mu \mathrm{m}$ is not seen in the astrophysical spectrum. The infrared spectrum of the $\mathrm{OH}$ maser. source is the first one in which structure has been reported in the $10 \mu \mathrm{m}$ feature. It is not known whether this unusual spectrum is due to a complicated radiative transfer process in the circumstellar shell or to the presence of a different absorbing material. If a different material is involved, it might bear some similarity in mineralogy to Juvinas. The absorption spectrum of the lunar soil sample \#6770I with its strong absorption peak near Ilum bears litcle resemblance to the astrophysical 


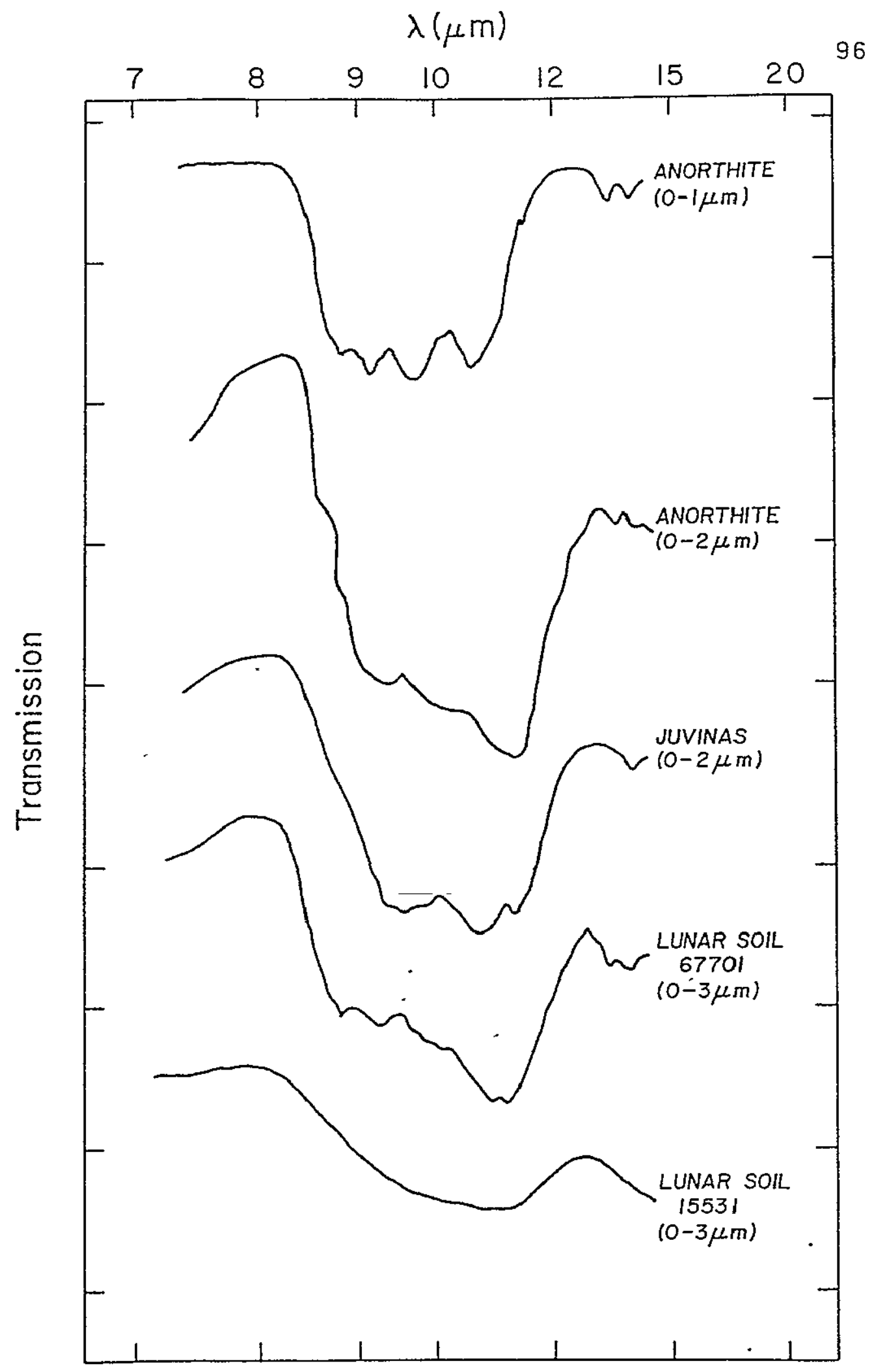

Fig. 20. Laboratory absorption spectra of anorthite, Juvinas and Iunar soils. 


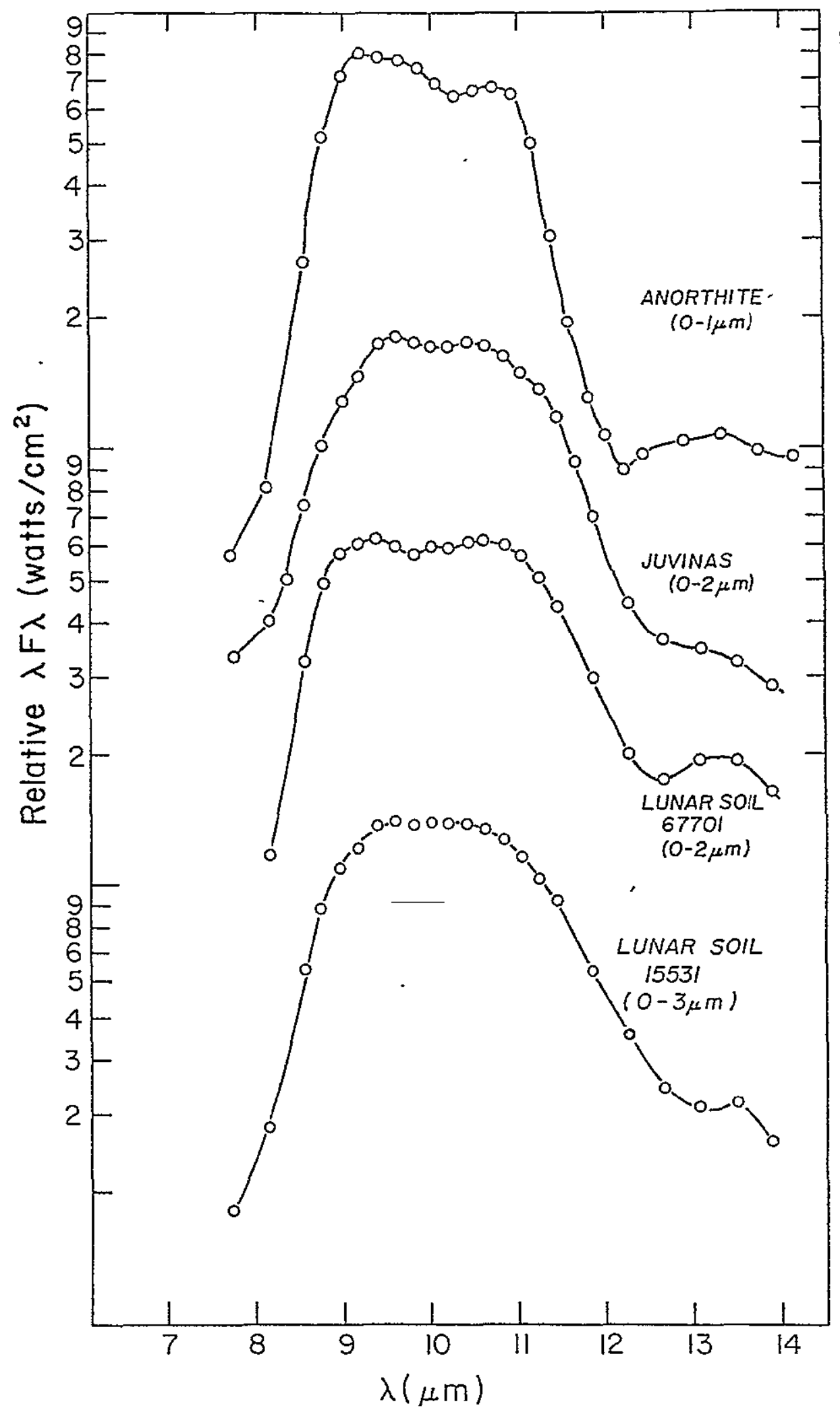

Fig. 21. Laboratory emission spectra of anorthite, Juvinas and lunar soils. 
absorption features even though in emission this sample looks somewhat similar to the Comet Kohourek spectrum. The 67701 absorption spectrum shows less structure than does the terrestrial anorthite spectrum although it is highly anorthositic in composition. Presumably this is due to a glassy or amorphous composition caused by proton or micrometeorite bombardment. The absorption spectrum of the 0-3um Iunar mare basalt (\#15531) is smoothly varying with a peak at 10.8um. This does not match the $9.8 \mu \mathrm{m}$ absorption peak of astrophysical spectra.

Figures 22 and 23 show laboratory absorption and emission spectra of glassy materials. These samples were prepared by heating them to their melting points and quenching in air. The resulting amorphous material was ground and sized as outlined in the section on sample preparation. The heating and quick cooling destroys periodicity in the material. - Strong absorption bands which appear as structure in the absorption features of the crystalline samples have been broadened until they are no longer resolved (Hunt and Salisbury 1974). For the albite and anorthite samples, the absorption features of the melted samples have shifted slightly to longer 
wavelengths, but for the slag there is a shift of the absorption feature to shorter wavelengths. Since the absorption and emission features of astrophysical objects are generally smooth and without structure, it is of particular interest to note the disappearance of structure in the emission and absorption features of materials which have been made amorphous by melting and air quenching. This seems to point to some type of astrophysical formation process in which grains would form with very little structure on crystallinity. Melted anorthite yields a Iaboratory emission spectrum (see Figure 16) that is quite similar to the spectrum of the irradiated sample, thus strengthening the conclusion that heating due to radiation destroys onder on periodicity in the material. In emission, the melted anorthite spectrum fits the short wavelength side of the Comet Kohoutek feature while the melted slag spectrum fits the Iong wave side, suggesting that some mixture of these glassy materials would also give a good fit. 


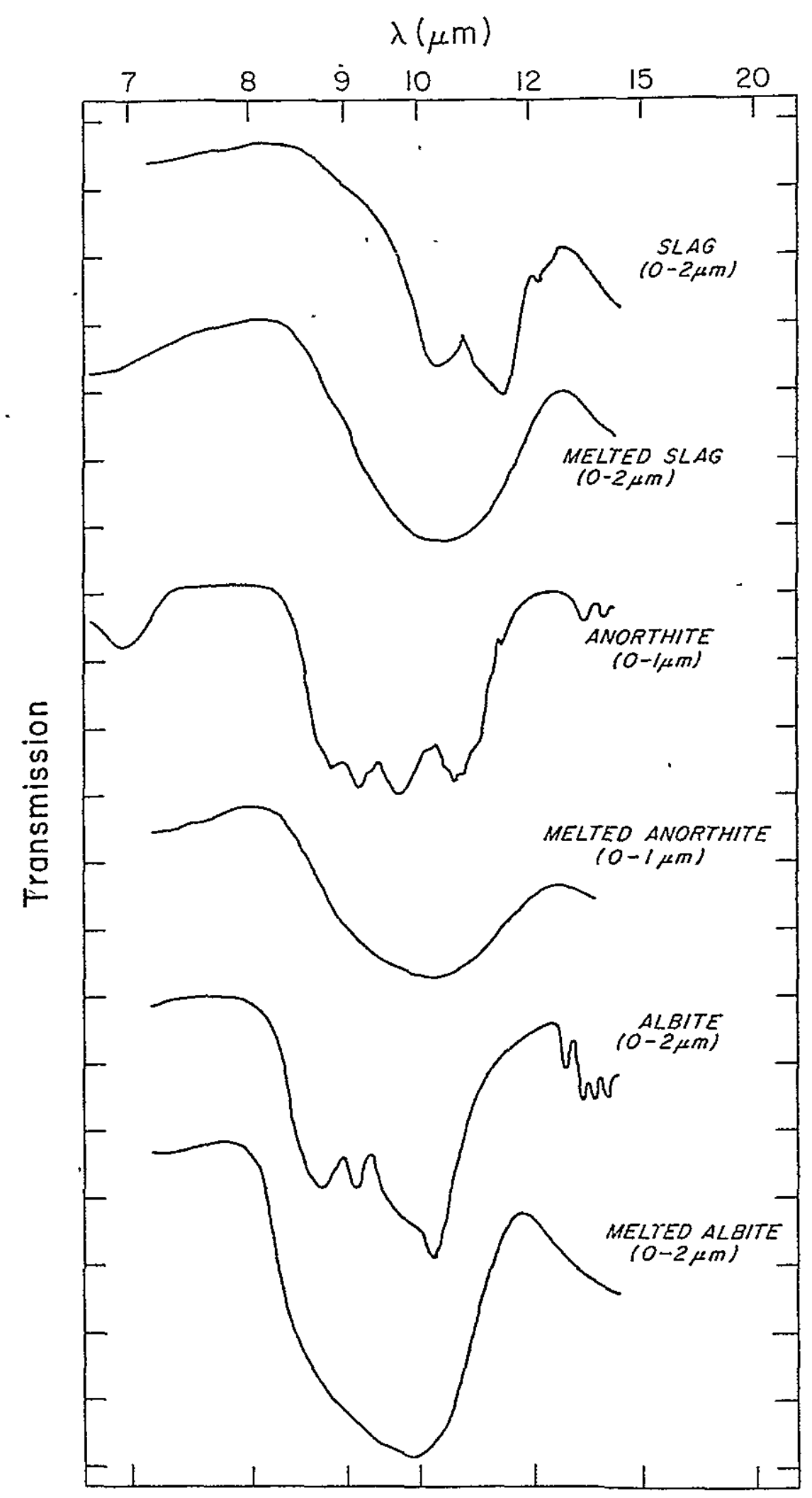

Fig. 22. Laboratory absorption spectra of melted and unmelted silicates. 


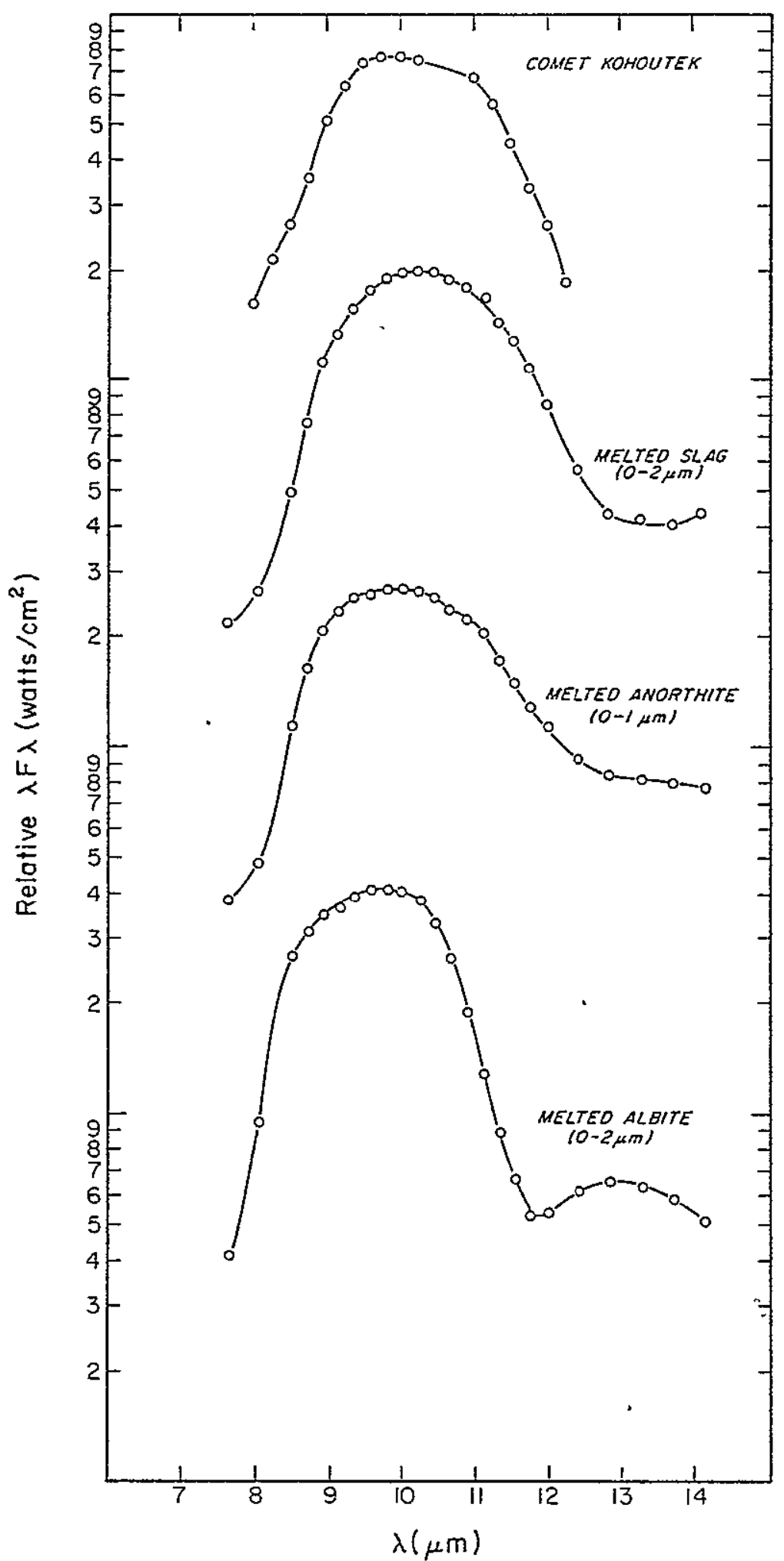

101

$\lambda(\mu \mathrm{m})$

Fig. 23. Laboratory emission spectra of melted glassy silicates. 


\section{SUMMARY AND CONCLUSIONS}

Laboratory infrared emission and absorption spectra have been taken of terrestrial silicates, meteorites and lunar soils in the form of micrometer and sub-micrometer grains. The emission spectra were taken in a way that imitates telescope observations. The purpose was to see which materials best simulate the $10 \mu \mathrm{m}$ astrophysical feature.

-Four terrestrial silicates and two meteorites were examined using broadband photometry. The emission spectra of forsterite, fayalite and Allende give a good fit to the $10 \mu \mathrm{m}$ broadband emission feature of Comets Bennett and Kohoutek. Quartz can be ruled out as a major component of cometary grains since its silicate feature occurs near $9 \mu \mathrm{m}$ and does not match the astrophysical emission feature. A study of the effect of grain size on the presence of the $10 \mu \mathrm{m}$ emission feature of forsterite shows that for particles larger than $37 \mu \mathrm{m}$ no feature is seen, but the feature is seen for particles smaller than this.

Intermediate resolution emission spectroscopy was used to study a group of 9 terrestrial silicates, 1 synthetic silicate, 6 meteorites and 2 lunar soils; 
form around late type stars. The identification of silicates as the material responsible for the $10 \mu \mathrm{m}$ astrophysical feature was called premature and alternate explanations were proposed. By 1974, when this project began, perhaps most research workers in infrared astronomy felt that the loum astrophysical feature was due to the presence of silicates. However; a labonatory study that simulated certain aspects of the astrophysical environment and that obtained emission spectra in a manner similar to the way celescope observations are made was needed. This work provides additional evidence in support of the silicate hypothesis by demonstrating that silicates with certain compositions, structure and grain sizes simulate the infrared emission spectra of comets. 
APPENDIX A

HANDLING SPECTROMETER DATA

This appendix gives in some detail the procedure used in handling the data from a laboratory run using the spectrometer filter wheel. Data for a sample of melted anorthite is tabulated and the steps are shown which proceed from raw data taken from the chart recorder to the finished product; vis., an emission spectrum of the grains plotzed as $\lambda F_{\lambda}$ versus $\lambda$. Since the data reduction was similar for all samples the chart recorder outputs and tabulations are given only for the one sample which is discussed here.

For each run it was necessary to view a calibration blackbody. All laboratory voltages were referenced to the blackbody which served the same purpose as a calibration star for an astronomer. Figures $A-1$ and $A-2$ show tracings of the chart recorder curves obtained when a blackbody was viewed.

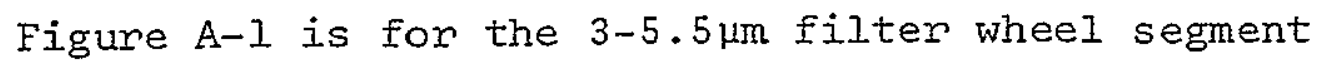
with wavelengths increasing to the left. Output voltage signal levels are shown on the vertical axis and the overall system gain was as indicated. Reference angles were read from a dial attached to 


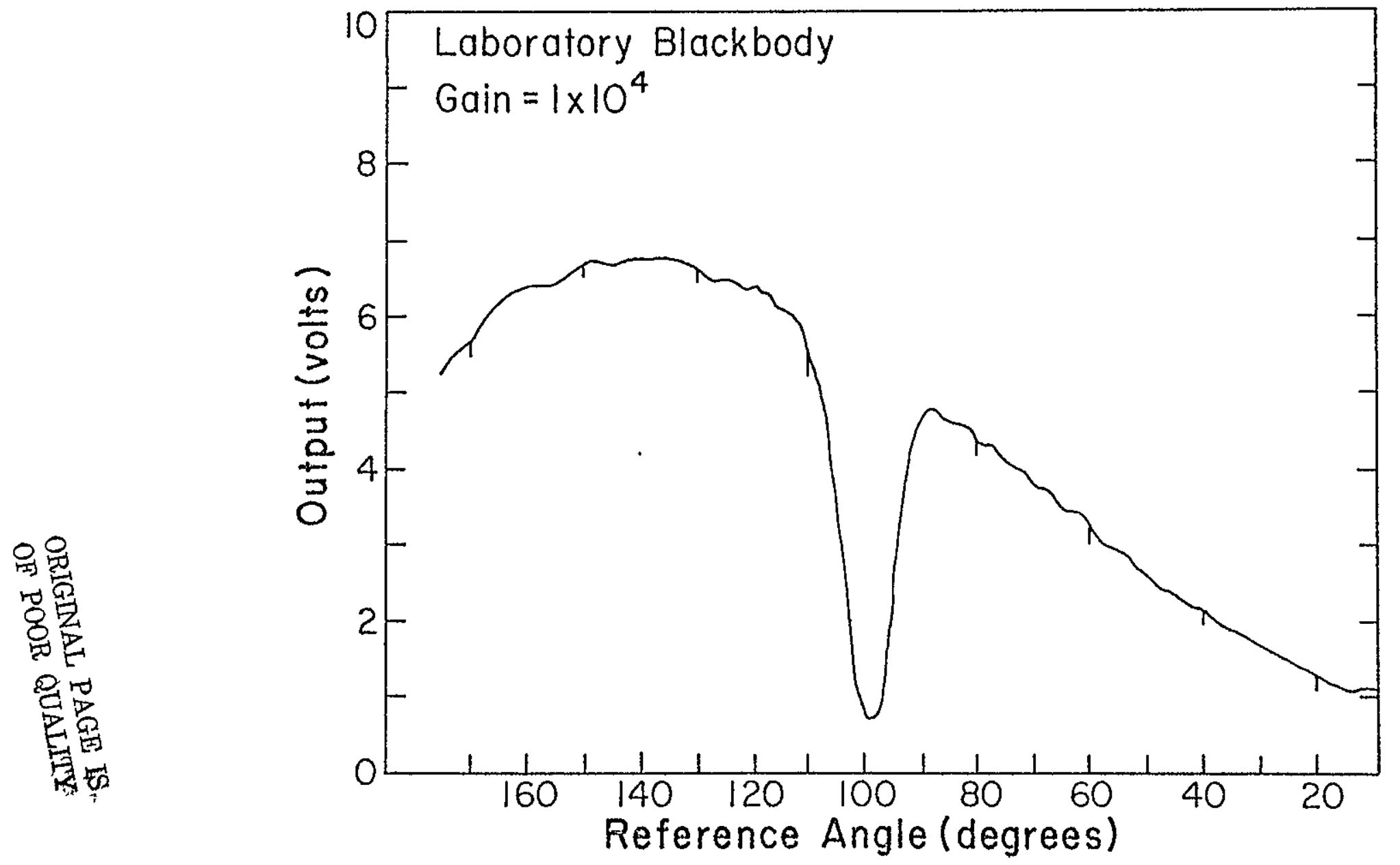

Fig. A-1. Chart recorder output of $550^{\circ} \mathrm{K}$ laboratory blackbody in the $3-5.5 \mu \mathrm{m}$ wavelength
range with $\lambda$ increasing to the left. 


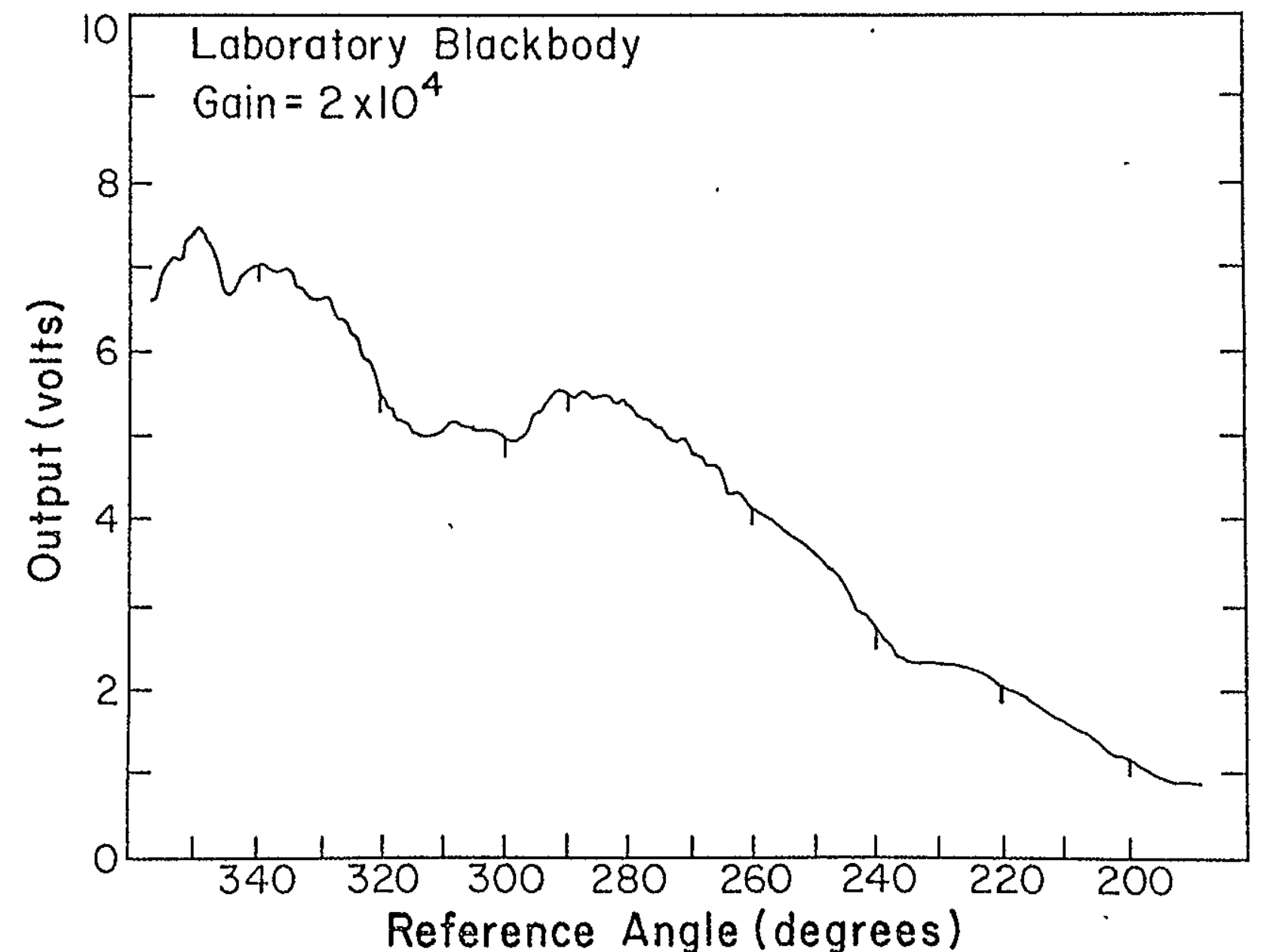

Fig. A-2. Chart reconder output of $550^{\circ} \mathrm{K}$ laboratory blackbody in the 7-14um wavelength range with $\lambda$ increasing to the right. 
the same shaft that supported the filter wheel.*

The large absorption feature near 100 degrees

$(4.3 \mu \mathrm{m})$ is due to atmospheric $\mathrm{CO}_{2}$. Figure A-2 shows the chart recorder output of the laboratory blackbody for the 7-14um filter wheel segment with wave-. length increasing to the right as reference angle decreases. The absorption feature in the 320-300 degree range is due possibly to atmospheric ozone. To use the spectrometer filter wheel it was necessary to know the transmission wavelength as a function of the filter wheel angle setting. To get this, a polystyrene film was inserted in the optical path during a run when viewing the laboratory blackbody. The wavelengths of the polystyrene spectral features are known and by putting reference angle marks on the chart recorder output, a relation between wavelength and filter wheel position can be found. Figures $A-3$ and $A-4$ show the chart recorder outputs having wavelength and angle information. These curves are the blackbody curves with the polystyrene absorption features superimposed. Tabulations

*For convenience the reference angles were read from a reference point that was 180 degrees away from the normal reference mark. The vertical marks on the graphs indicate angle réference points made during a run. 


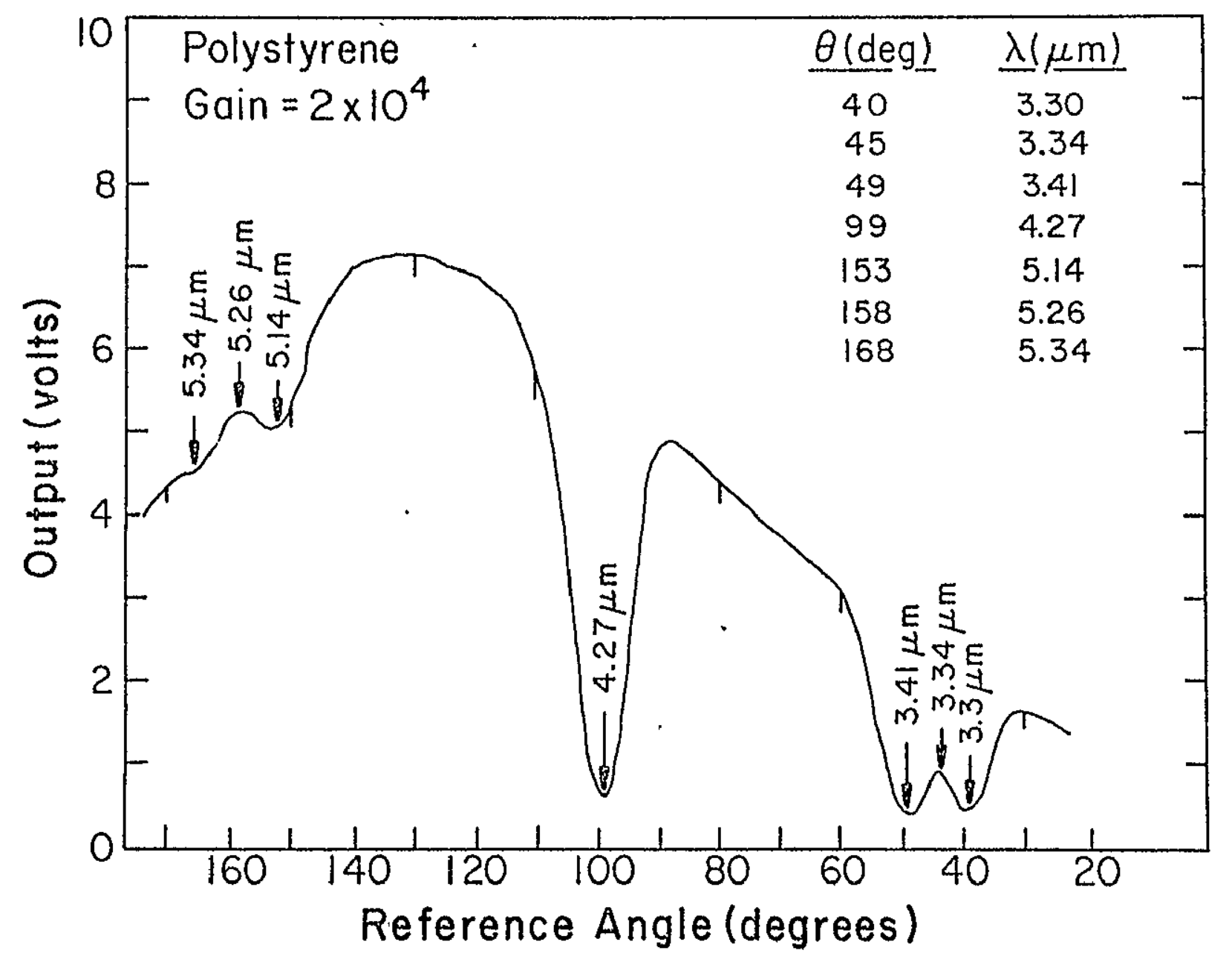
Fig. A-3. Chart recorder output of a wavelength calibration run in the $3-5.5 \mu m$ wave- $1-5$
lengths range using polystyrene film. The tabulation gives absorption feature wavelengths and angular positions. 

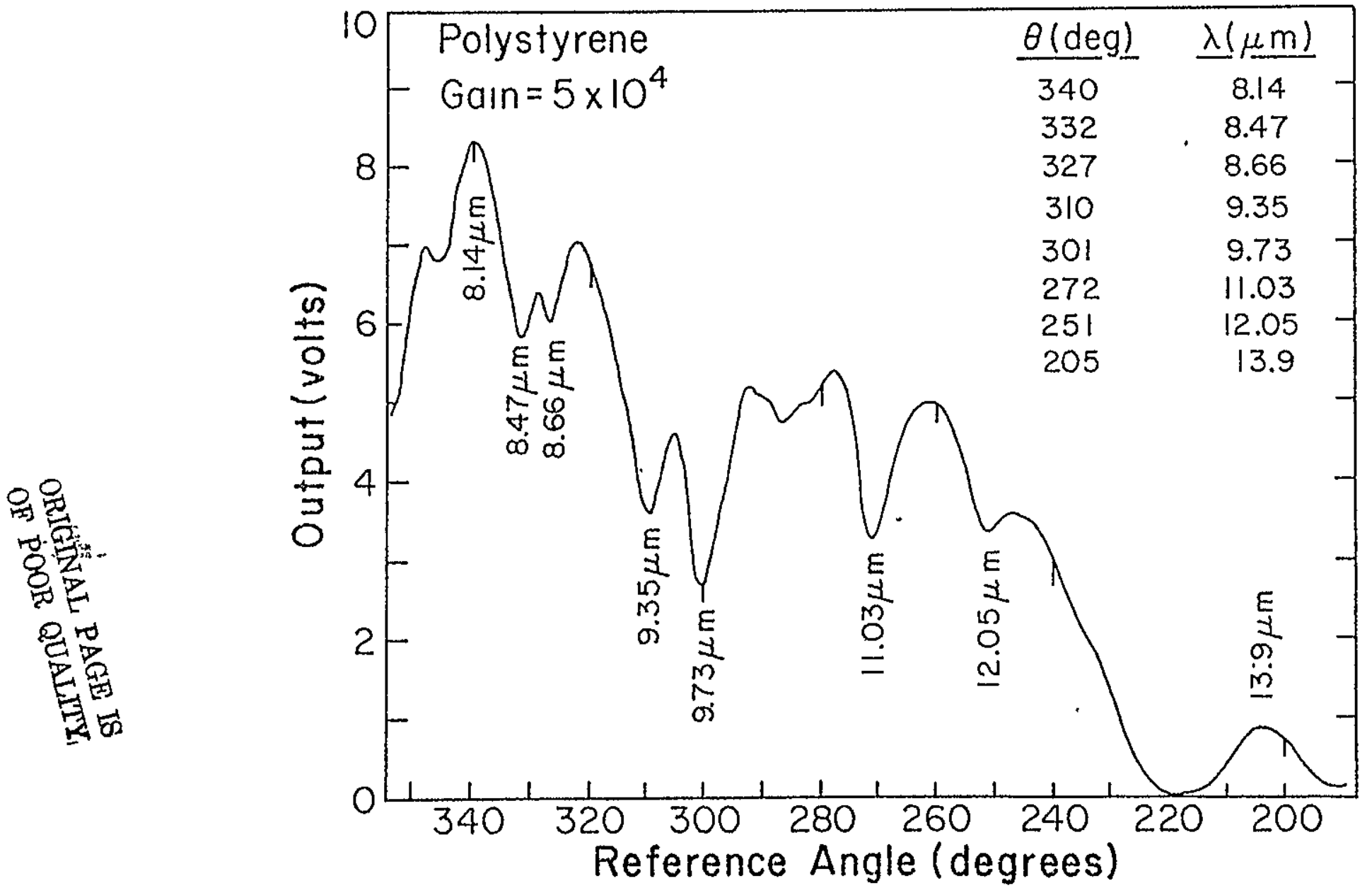

Fig. A-4. Chart recorder output of a wavelength calibration run in the $7-14 \mu m$ range using polystyrene film. The tabulation gives absorption feature wavelengths and their angular positions. 
of the angle-wavelength relations are given in the figures.

Figure A-5 shows a straight line fit to the $(\theta, \lambda)$ data points for the $3-5 \mu \mathrm{m}$ segment.

For the long wavelength segment, a least squares fit was made to the $(\theta, \lambda)$ data points and the following equation described the relation between wavelength in $\mu \mathrm{m}$ and angular position in degrees for the spectrometer filter wheel:

$$
\lambda=14.16-(\theta-200) \cdot(0.0434)
$$

for $200 \leq \theta \leq 350$.

A plot of the $(\theta, \lambda)$ data points and the least squares fit to these points is shown in Figure A-6. An angle calibration check was made at the beginning of each run. It is particularly important to make such a check following disassembly of the dewar since it is highly unlikely that in reassembly the reference angle marker would be returned to the exact position it had before.

Tracings of the chapt recorder outputs of a sample of melted anorthite are shown in Figures A-7 and A-8. Since anorthite has no spectral features in. the $3-5 \mu \mathrm{m}$ band its chart recorder curve has the same general shape as the laboratory blackbody curve; 


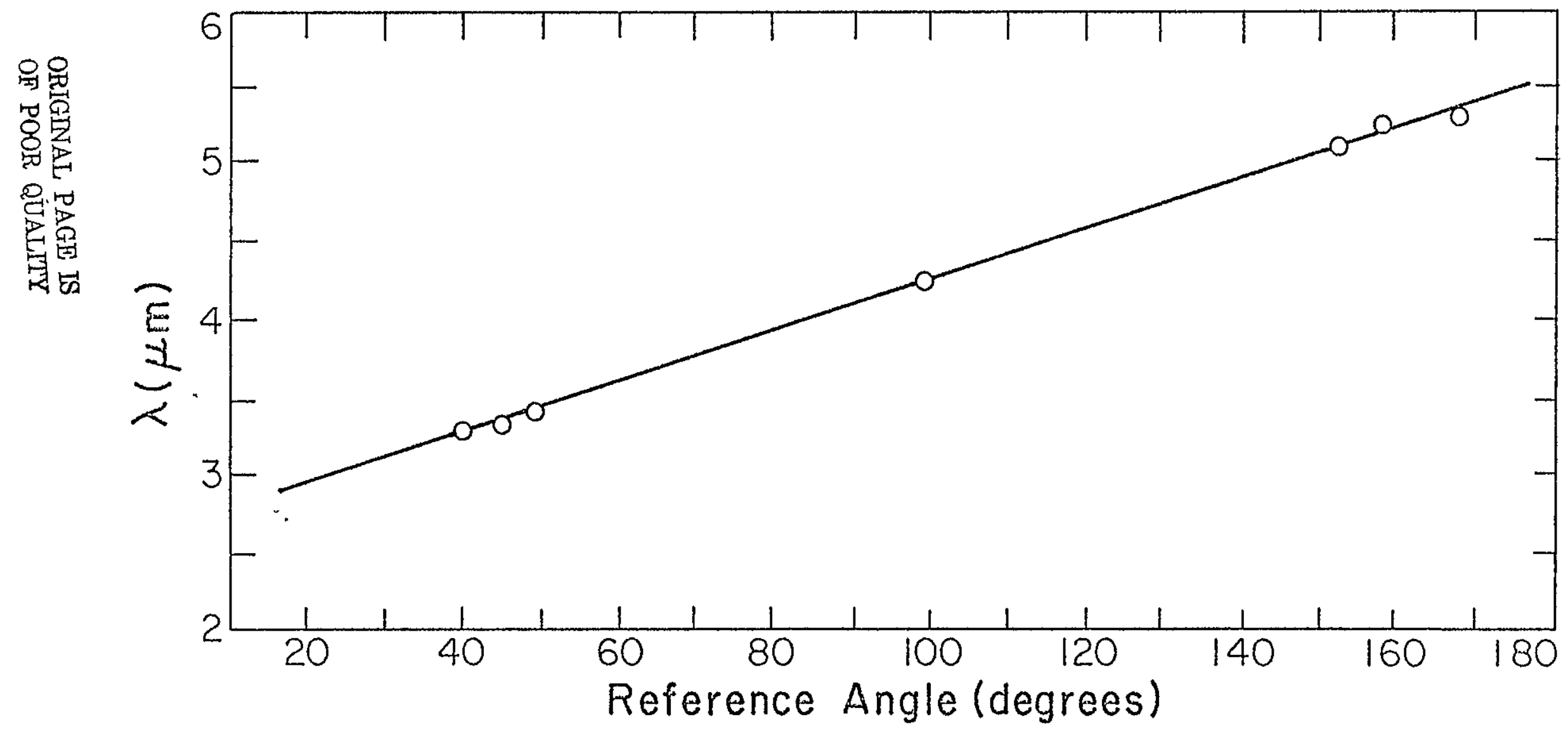

Fig. A-5. Wavelength versus reference angle plot of spectrometer filter wheel in the

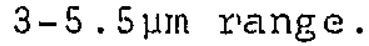




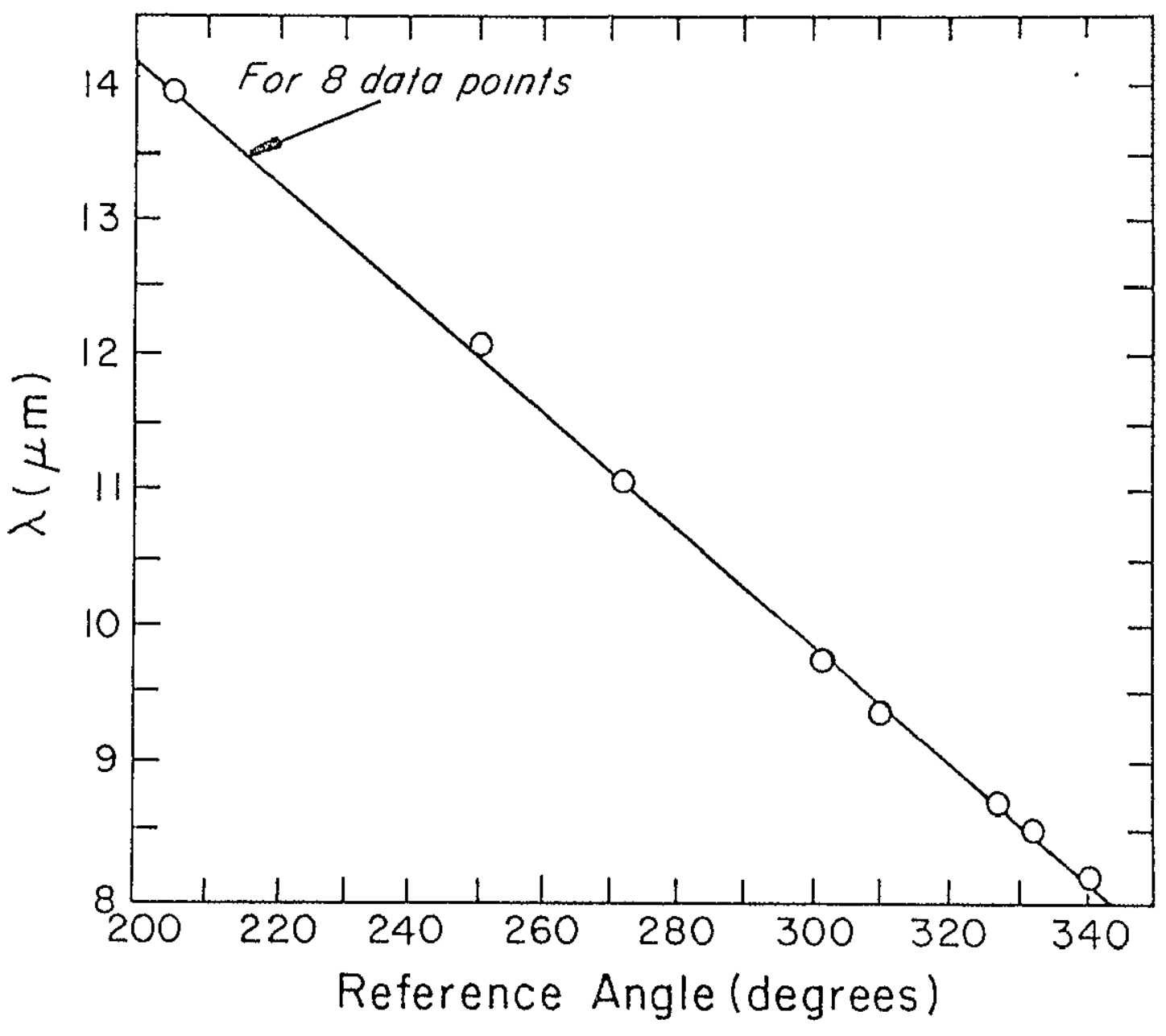

Fig. A-6. Wavelength versus reference angle plot of spectrometer filter wheel in $8-14 \mu \mathrm{m}$ range. 

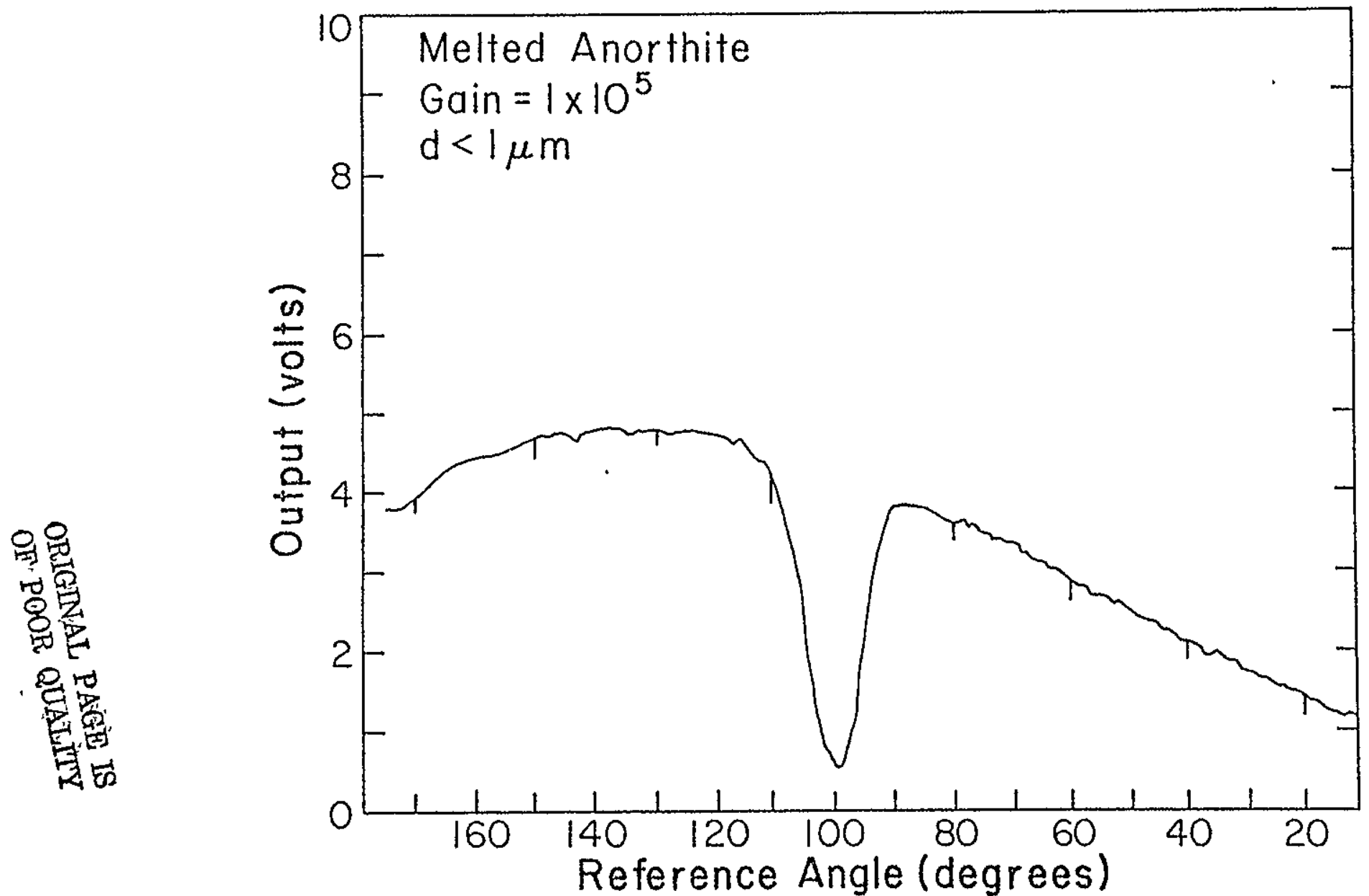

Fig. A-7. Chart recorder output of a melted anorthite sample in the $3-5.5 \mu m$ wavelength range. 


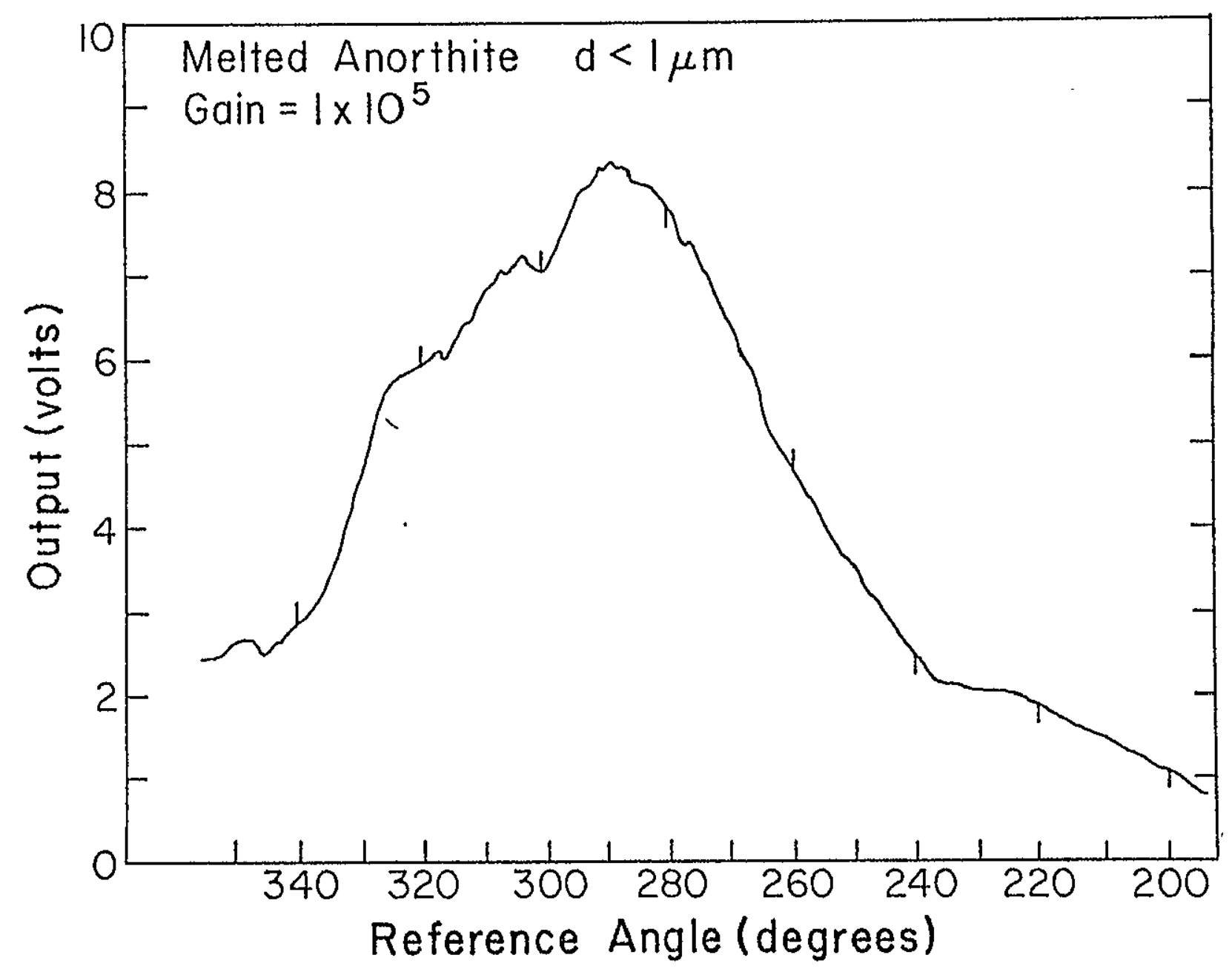

Fig. A-8. Chart recorder output of a melted anorthite sample in the 7-14um wavelength range. 
comparisons were made with the intermediate resolution spectra of Comet Kohoutek. Infrared absorption spectra were taken of selected materials from this group. The results were as follows: Type I carbonaceous chondrites and a laboratory amorphous silicate exhibit emission peaks near $9.8 \mu \mathrm{m}$, the wavelength of the emission maximum in astrophysical objects. The emission features of these "low temperature hydrous" materials are narrow compared to comet Kohoutek. The fine grain emission spectrum of Murray, a C2 meteorite which is a combination of hydrous layer lattice silicates and the high temperature materials olivine and pyroxene, is generally a good fit to the cometary spectrum except for some structure in the 10 um region. A lunar mare basalt and a basalatic achondrite similar to the lunar basalt in mineralogy yield emission spectra which are good fits to the Comet Kohourek emission spectrum.

Absorption spectra of these two samples, however, do not match astrophysical absorption features very well. Emission spectra of glassy olivine slag and glassy anorthite resemble the cometary emission feature strongly, although the peak emission for the olivine slag is near $10.2 \mu \mathrm{m}$ instead of $9.8 \mu$. 


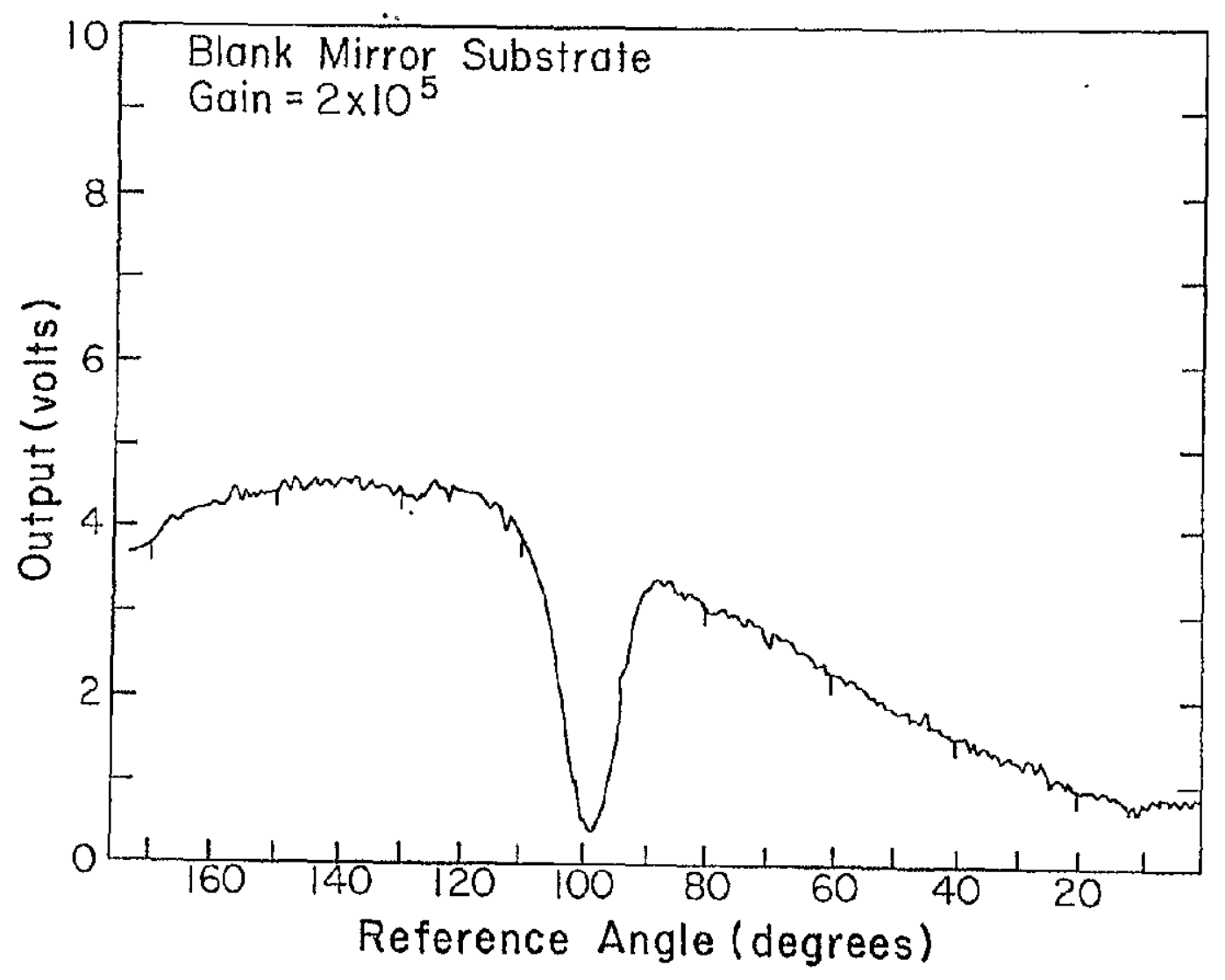

Fig. A-9. Chart recorder output of a mirror blank in 3-5.5um wavelength range. 


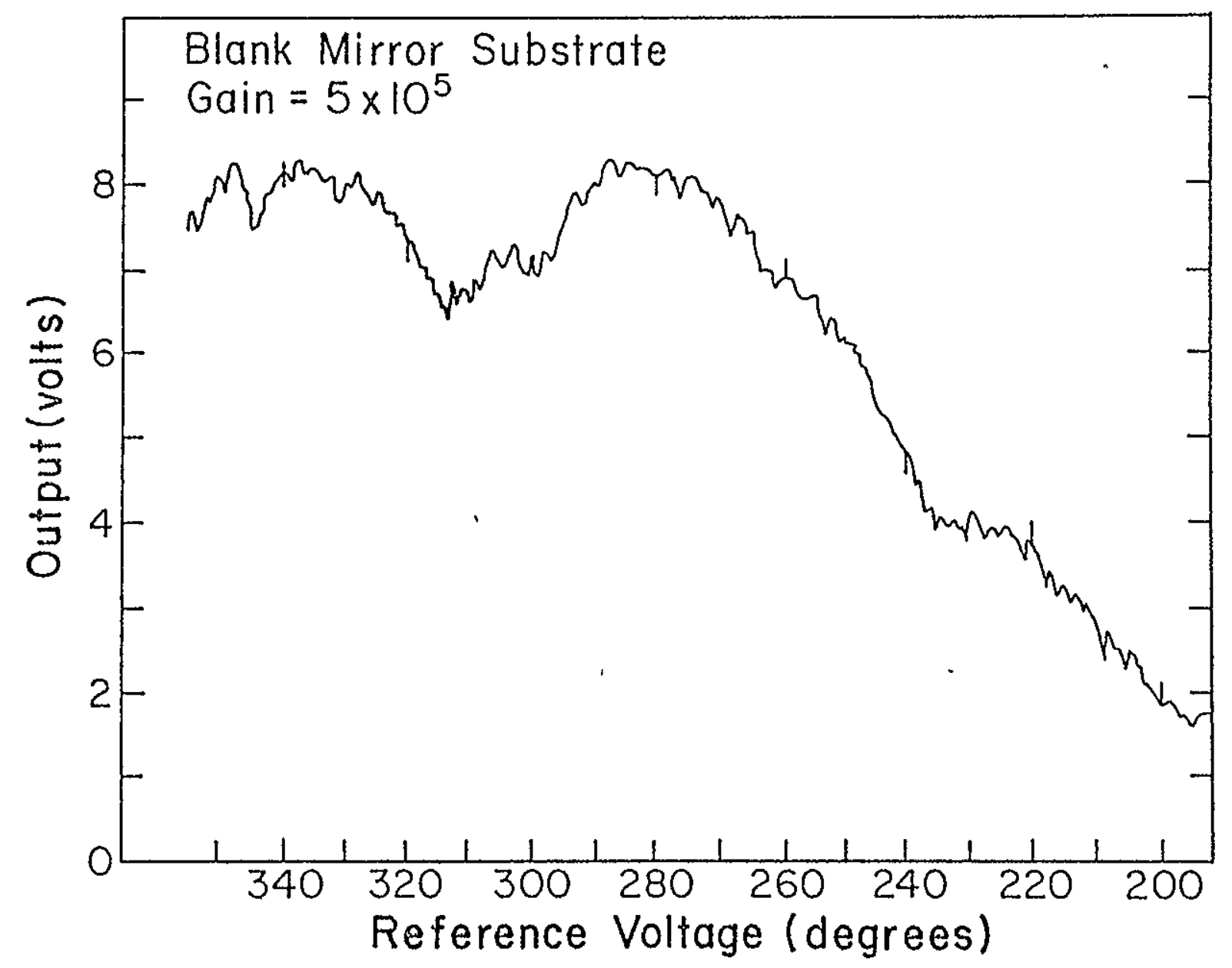

Fig, A-10. Chart recorder output of a mirror blank in the 7-14um wavelength range. 
shapes and signal levels of the curves for the upper and lower positions of the source were essentially the same except in the case of the mirror blank. For the mirror the slight excess signal noticed when the source was in the lower beam was not seen when the source was in the upper beam.

Table A-2 gives the calculated data for the melted anorthite sample. Column 2 gives the values of wavelength for the angles listed in column I calculated using equation $(A-1)$. Column 3 lists the calculated values of a normalized $\lambda B_{\lambda}$ function where $B_{\lambda}$ is the Planck function. This function was normalized to $I$ at $\lambda=6.67 \mu \mathrm{m}$, the peak wavelength for $\mathrm{T}=550^{\circ} \mathrm{K}$. Columns 4 and 6 give magnitudes of the melted anorthite sample and the mirror blank calculated using equation (4). The radiation due to the grains, the excess, can be obtained by either plotting the magnitude curves of the mirror and sample with respect to the normalized $\lambda \mathrm{B}_{\lambda}$ curve and subtracting one curve from another or by calculating the excess directly using the relation

$$
\begin{aligned}
& \qquad \begin{aligned}
E=\left(\lambda B_{\lambda}\right)_{n} \cdot \frac{V_{2}-V_{3}}{V_{I}} \\
\text { where }\left(\lambda B_{\lambda}\right)_{n}=a \text { normalized blackbody function } \\
V_{I}=\text { blackbody signal at wavelength } \lambda \\
\text { ORIGINAI PAGE IS } \\
\text { OF POOR QUAIIT }
\end{aligned}
\end{aligned}
$$




$$
\begin{aligned}
& \mathrm{v}_{2}=\text { melted anorthite sample signal } \\
& \mathrm{v}_{3}=\text { mirror signal }
\end{aligned}
$$

Column 5 gives the values of the infrared radiation of the grains calculated using equation (A-2). Figure A-Il, the final figure in this section, shows plots of $\lambda B_{\lambda}, \lambda F_{\lambda}$ of the anorthite grain sample and $\lambda F_{\lambda}$ of a blank substrate. The $\lambda F_{\lambda}$ of the anorthite grains alone is shown in Figure 23 .

TABLE A-I

DETECTOR SIGNAL VOLTAGES ( $\mu$ VOLTS)

$\mathrm{V}_{1}$ - Laboratory Blackbody

$\mathrm{V}_{2}$ - Melted Anorthite Sample

$\mathrm{V}_{3}$ - Blank Aluminum Mirror

$\begin{array}{llll}\text { Angle } & \frac{V_{I}}{110} & \frac{V_{2}}{I 9} & \frac{V_{3}}{6.0} \\ 200 & \begin{array}{lll}I 9 \\ 210\end{array} & 26 & 8.0 \\ 220 & 200 & 33 & 10.8 \\ 230 & 225 & 37 & 12.4 \\ 240 & 260 & 44 & 14.4 \\ 250 & 350 & 64 & 17.8 \\ 255 & 380 & 75 & 19.6 \\ 260 & 405 & 87 & 20.6 \\ 265 & 455 & 106 & 21.8 \\ 270 & 465 & 122 & 23\end{array}$


TABLE A-I (continued)

$\begin{array}{llll}\text { Angle } & V_{1} & V_{2} & V_{3} \\ 275 & 500 & I 37 & 24 \\ 280 & 530 & 149 & 24.4 \\ 285 & 535 & 156 & 24.8 \\ 290 & 540 & 159 & 24.6 \\ 295 & 515 & 150 & 23.2 \\ 300 & 485 & 138 & 22.2 \\ 305 & 505 & 138 & 23 \\ 310 & 500 & 130 & 21.8 \\ 315 & 500 & 119 & 2 I .8 \\ 320 & 545 & 116 & 23.4 \\ 325 & 620 & 107 & 25.6 \\ 330 & 655 & 85 & 26.8 \\ 340 & 700 & 53 & 27.6 \\ 350 & 750 & 49 & 28\end{array}$


TABLE A-2

MAGNITUDE RESULTS OF MELTED ANORTHITE

$$
\begin{aligned}
\left(\lambda B_{\lambda}\right)_{n}= & \text { normalized reference function } \\
M_{2}= & \text { magnitude of melted anorthite sample with } \\
& \text { respect to the laboratory blackbody } \\
E= & \text { relative } \lambda F_{\lambda} \text { of the anorthite grains. } \\
M_{3}= & \text { magnitude of blank mirror with respect to } \\
& \text { the laboratory blackbody }
\end{aligned}
$$

\begin{tabular}{|c|c|c|c|c|c|}
\hline $\begin{array}{c}\text { Angle } \\
\text { (degrees) }\end{array}$ & $\begin{array}{c}\lambda \\
(\mu \mathrm{m})\end{array}$ & $\left(\lambda B_{\lambda}\right)_{n}$ & $M_{2}$ & $E \times 10^{2}$ & $M_{3}$ \\
\hline 200 & 14.16 & .456 & 1.91 & 5.39 & 3.16 \\
\hline 210 & 13.72 & .482 & 1.94 & 5.60 & 3.22 \\
\hline 220 & 13.29 & .510 & 1.96 & 5.66 & 3.17 \\
\hline 230 & 12.85 & .539 & 1.96 & 5.90 & 3.15 \\
\hline 240 & 12.42 & .570 & 1.93 & 6.49 & 3.14 \\
\hline 250 & 11.99 & .603 & 1.84 & 7.96 & 3.23 \\
\hline 255 & 11.77 & .620 & 1.76 & 9.04 & 3.22 \\
\hline 260 & 11.55 & .638 & 1.67 & 10.46 & 3.23 \\
\hline 265 & 11.34 & .656 & 1.58 & 12.13 & 3.30 \\
\hline 270 & 11.12 & .674 & 1.45 & 14.35 & 3.26 \\
\hline 275 & 10.90 & .692 & 1.41 & 15.65 & 3.30 \\
\hline 280 & 10.68 & $.7 I I$ & 1.38 & 16.72 & 3.34 \\
\hline 285 & 10.47 & .731 & 1.34 & 17.92 & 3.33 \\
\hline 290 & 10.25 & .750 & 1.33 & 18.67 & 3.35 \\
\hline 295 & 10.03 & .770 & 1.34 & 18.95 & 3.37 \\
\hline 300 & 9.82 & .789 & 1.36 & 18.85 & 3.35 \\
\hline
\end{tabular}




\section{TABLE A-2 (continued)}

\begin{tabular}{|c|c|c|c|c|c|}
\hline $\begin{array}{c}\text { Angle } \\
\text { (degrees) } \\
\end{array}$ & $\begin{array}{c}\lambda \\
(\mu \mathrm{m}) \\
\end{array}$ & $\left(\lambda B_{\lambda}\right)$ & $\mathrm{M}_{2}$ & $\operatorname{Ex} 10^{2}$ & $\mathrm{M}_{3}$ \\
\hline 305 & 9.60 & .809 & 1.41 & 18.43 & 3.35 \\
\hline 310 & 9.38 & .829 & 1.46 & 17.94 & 3.40 \\
\hline 315 & 9.16 & .849 & 1.56 & 16.50 & 3.40 \\
\hline 320 & 8.95 & .868 & 1.68 & 14.75 & 3.42 \\
\hline 325 & 8.73 & .887 & 1.91 & 11.65 & 3.46 \\
\hline 330 & 8.51 & $.90^{\prime} 5$ & 2.22 & 8.04 & 3.47 \\
\hline 340 & 8.08 & .939 & 2.80 & $3.4 I$ & $3.5 I$ \\
\hline 350 & 7.65 & .968 & 2.96 & 2.7 & 3.57 \\
\hline
\end{tabular}


125

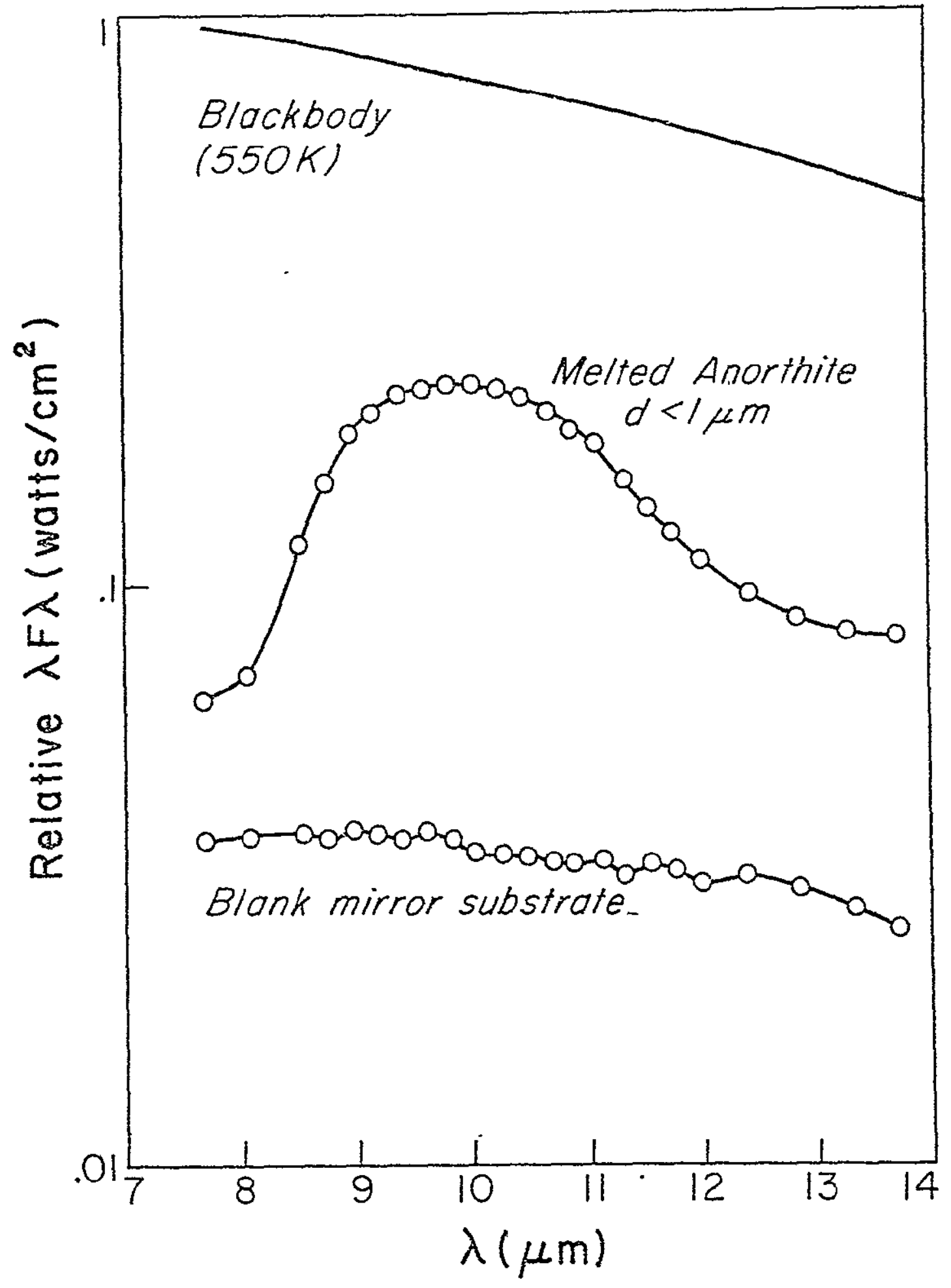

Fig. A-11. Laboratory emission spectra of a melted anorthite sample and a mirror substrate using intermediate resolution spectroscopy. 


\section{APPENDIX B: A THIN FILYY EXPERIMENT}

Before experimental work with grain samples was undertaken, it was thought that a measurement of the strength of the Si-0 absorption at loum using thin films would be useful as an aid in determining the sizes of grains with which to work. Silicon monoxide was chosen as a thin film material which would exhibit an absorption near loum. A series of si-0 films on $\mathrm{KBr}$ disks were prepared using a vacuum system with a tungsten filament heating element and a Sloan Thickness Gauge Monitor. FigureB-I shows the transmission of a $5100 \AA \mathrm{Si}-\mathrm{O}$ film on $\mathrm{KBr}$ as measured in the Dual Beam Perkin Elmer Model 521 spectrophotometer.

The strength of the loum absorption feature was calculated as the ratio of the transmission at $10 \mu \mathrm{m}$ to the transmission at $7 \mu \mathrm{m}$ where it was assumed there would be no absorption. Table BI gives the list of thin films and the percent transmission of each. The transmission through a thin film can be written as

$$
T=I / I_{0}=e^{-\tau}
$$

where $T$ is the fractional transmission and $\tau$ is the optical depth of the film. A semilog plot of the data from Table $B-1$ is shown in Figure B-2 showing that the relation $I=I_{0} e^{-\tau}$ is obeyed fairly well. Equation 
B-I can be written as

$$
\tau=\operatorname{X\rho t}=-\ln \left(I / I_{0}\right)
$$

where $X$ and $\rho$ have the same definitions as given on page 11 and $t$ is the film thickness in $\mathrm{cm}$. The density of silicon monoxide is $2.1 \mathrm{gm} / \mathrm{cm}^{3}$. For two films of thicknesses $t_{1}$ and $t_{2}$,

$$
\begin{aligned}
\operatorname{Xp}\left(t_{2}-t_{1}\right) & =\ln \left(I_{1} / I_{0}\right)-\ln \left(I_{2} / I_{0}\right) \\
& =\ln \left(T_{1} / T_{2}\right) .
\end{aligned}
$$

Using the data points $(0,1)$ and $\left(5.1 \times 10^{-5}, .45\right)$, the value of the mass absorption coefficient is

$$
\begin{aligned}
x & =\frac{\ln (1 / .45)}{5.1 \times 10^{-5}} \cdot \frac{1}{2.1} \\
& =7.5 \times 10^{3} \mathrm{~cm}^{2} / \mathrm{gm} .
\end{aligned}
$$

This thin film result agrees fairly well with the value of $7.7 \times 10^{3} \mathrm{~cm}^{2} / \mathrm{gm}$ obtained by Hass and Salzburg (1954) who used a similar technique and falls within the range of mass absorption coefficients of powdered silicates as reported by Day et al. (1974) and Hunt et al. (1950). 


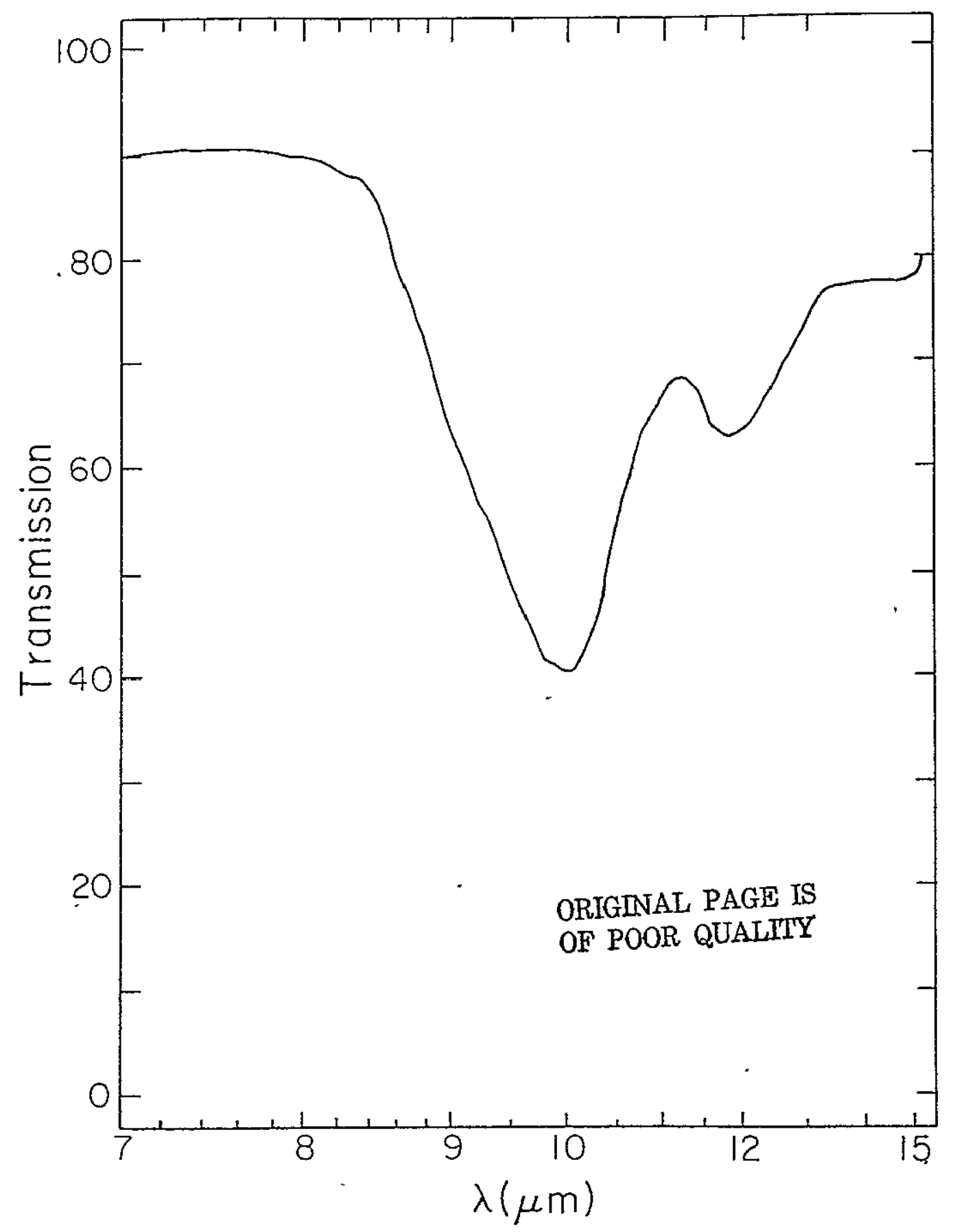

Fig. B-I. Transmission of a $5100 \AA$ sio thin film. 
TABLE $B-1$

LIST OF SIO DEPOSITIONS

\% Transmission

$\begin{array}{cc}\text { FiIm Thickness }(\stackrel{\circ}{\AA}) & \frac{\left(I_{10 \mu \mathrm{m}} / I_{7 \mu \mathrm{m}}\right)}{575} \\ 2000 & 86 \\ 3700 & 69 \\ 5100 & 58 \\ & 45\end{array}$




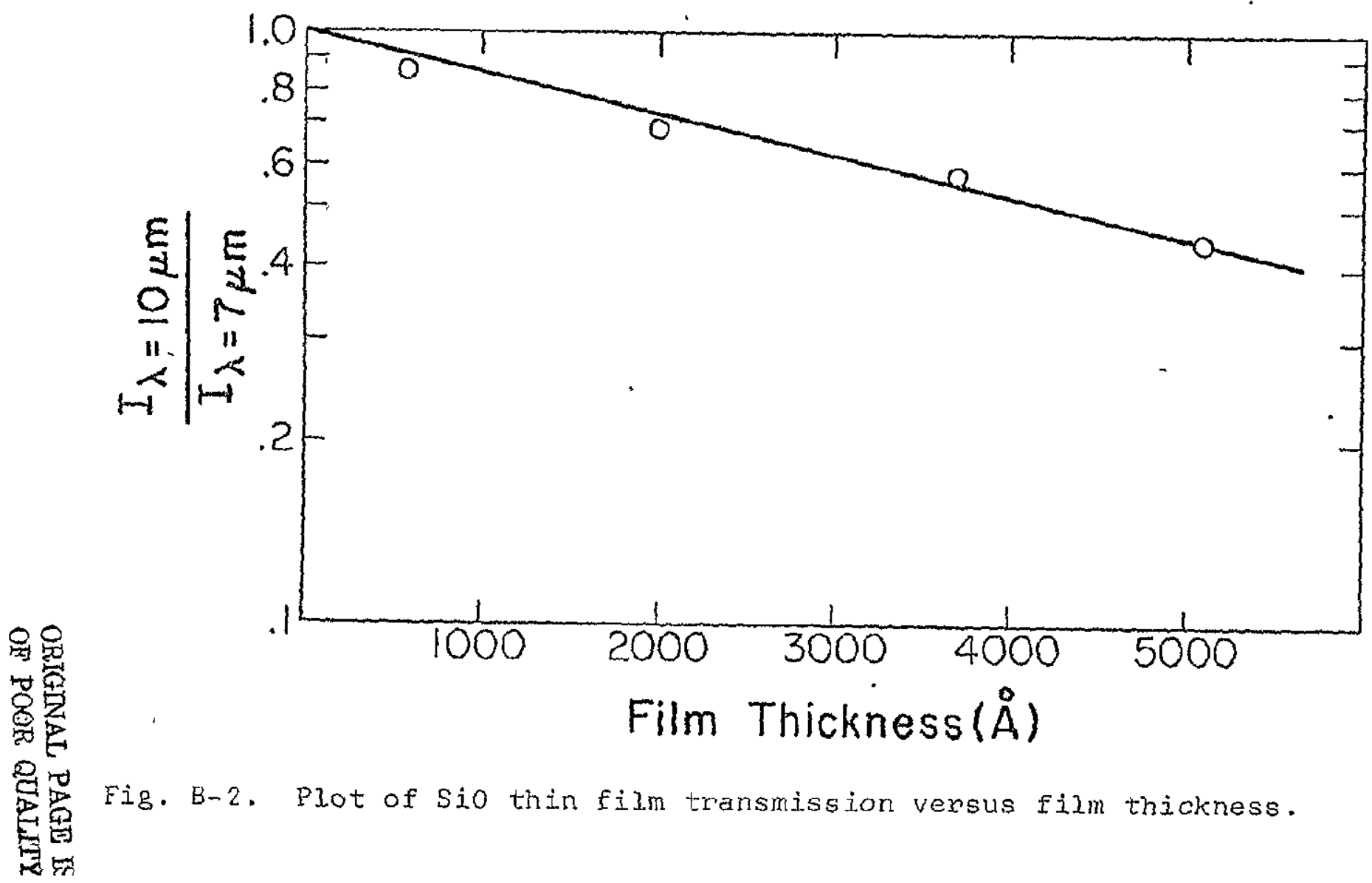


APPENDIX C: NORMATIVE MINERAL COMPOSITIONS OF CERTAIN SAMPLES

The chemical analyses of rock samples are usually given in terms of oxides. It is userul to know the rock composition in terms of minerals. This appendix gives a hypothetical mineralogy, the CIPW mineral norms, of several samples used in this study based on their chemical analyses. These mineral norms were supplied by Claus Shultz of the University of Minnesota. The reader should consult Table 1 for chemical formulas not given in this appendix.

Sample: COLUMBIA RIVER BASALT (BCR-I)

\begin{tabular}{|c|c|c|c|}
\hline Oxide & Wt. Percent & $\begin{array}{l}\text { Normative } \\
\text { Mineral }\end{array}$ & Wt. Percent \\
\hline $\mathrm{SiO}_{2}$ & 54.5 & Quartz & 8.66 \\
\hline $\mathrm{Fe}_{2} \mathrm{O}_{3}$ & 3.70 & Albite & 28.25 \\
\hline MgO & 3.3 & Diopside & 12.49 \\
\hline $\mathrm{Na}_{2} \mathrm{O}$ & 3.3 & $\underset{\left(\mathrm{Fe}_{3} \mathrm{O}_{4}\right)}{\text { Magnetite }}$ & 5.43 \\
\hline $\begin{array}{l}\mathrm{TiO}_{2} \\
\mathrm{MnO}\end{array}$ & $\begin{array}{l}2.2 \\
.17\end{array}$ & $\mathrm{Ca}_{5}(\mathrm{~F}, \mathrm{Cl}, \mathrm{OH})\left(\mathrm{PO}_{4}\right)_{3}$ & .85 \\
\hline $\mathrm{Al}_{2} \mathrm{O}_{3}$ & 13.70 & $\begin{array}{l}\text { Orthoclase } \\
\mathrm{K}\left(\mathrm{AlSi}_{3} \mathrm{O}_{8}\right)\end{array}$ & 10.16 \\
\hline CaO & 7.00 & Anorthite & 17.76 \\
\hline $\mathrm{K}_{2} \mathrm{O}$ & 1.7 & Hypersthene & 12.17 \\
\hline $\mathrm{P}_{2} \mathrm{O}_{5}$ & .36 & $\begin{array}{l}\text { Imenite } \\
\text { FeTiO }_{3}\end{array}$ & 4.23 \\
\hline
\end{tabular}


Sample: LAKE SUPERIOR DIABASE

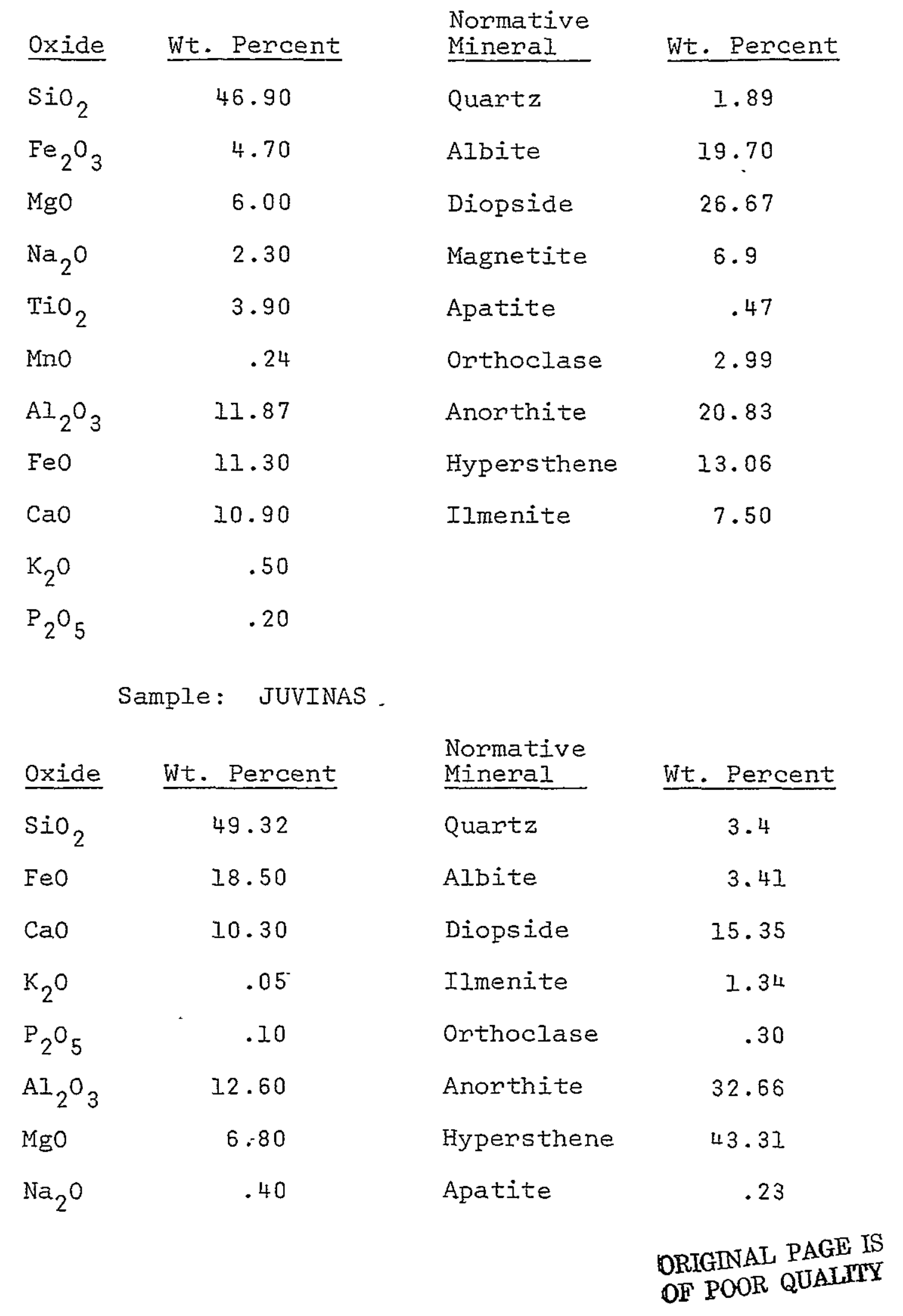


Sample: JUVINAS (con't)

\begin{tabular}{|c|c|c|c|}
\hline Oxide & Wt. Percent & $\begin{array}{l}\text { Normative } \\
\text { Mineral }\end{array}$ & Wt. Percent \\
\hline $\mathrm{TiO}_{2}$ & .70 & & \\
\hline $\mathrm{MnO}$ & .53 & & \\
\hline
\end{tabular}

Sample: LUNAR MARE BASALT (\#I553I)

$\begin{array}{lcllc}\text { Oxide } & \text { Wt. Percent } & & \begin{array}{l}\text { Nonmative } \\ \text { Mineral }\end{array} & \text { Wt. Percent } \\ \mathrm{SiO}_{2} & 46.40 & & \text { Orthoclase } & 5.25 \\ \mathrm{FeO} & 20.70 & \text { Anorthite } & 22.71 \\ \mathrm{CaO} & 9.38 & \text { Diopside } & 19.35 \\ \mathrm{~K}_{2} \mathrm{O} & .90 & \text { Olivine } & 18.34 \\ \mathrm{MnO} & .25 & \text { Albite } & 2.51 \\ \mathrm{Al}_{2} \mathrm{O}_{3} & 9.90 & \text { Hypersthene } & 27.72 \\ \mathrm{MgO} & 11.30 & \text { IImenite } & 4.12 \\ \mathrm{Na}_{2} \mathrm{O} & .30 & & \\ \mathrm{TiO}_{2} & 2.20 & & \end{array}$

Sample: LUNAR HIGHLAND SOIL ( \#67700)

\begin{tabular}{lcllc} 
Oxide & Wt. Percent & & $\begin{array}{l}\text { Normative } \\
\text { Mineral }\end{array}$ & Wt. Percent \\
\cline { 3 - 4 } $\mathrm{SiO}_{2}$ & 44.80 & & Albite & 4.22 \\
$\mathrm{FeO}$ & 4.20 & & Diopside & 6.63 \\
$\mathrm{CaO}$ & 16.87 & Olivine & 8.05 \\
$\mathrm{TiO}_{2}$ & .44 & Anorthite & 75.32 \\
$\mathrm{Al}_{2} \mathrm{O}_{3}$ & 28.50 & Hypersthene & 4.95
\end{tabular}




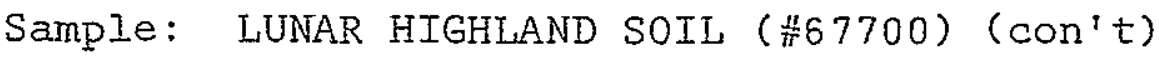

\begin{tabular}{|c|c|c|c|}
\hline Oxide & Wt. Percent & $\begin{array}{l}\text { Normative } \\
\text { Mineral }\end{array}$ & Wt. Percent \\
\hline $\mathrm{MgO}$ & 4.90 & IImenite & .83 \\
\hline $\mathrm{Na}_{2} \mathrm{O}$ & .50 & & \\
\hline Mno & .06 & & \\
\hline
\end{tabular}

ORIGINAL PAGE IS OF POOR QUALITY 


\section{REFERENCES}

Aannestad, P. A. 1975, Ap. J. 200, 30.

Adams, J. B. 1976, Private Communication.

Aiken, D. K. and Jones, B. 1973, Ap. J. 184, 127.

Anderson, D. L. 1973, in Cosmochemistry, A. G. W. Cameron, Editor (D. Reidel Publ. Co., Boston), p. 127.

Arrhenius, G. and Alfven, H. 1971, Earth and Planetary Space Science Letters 10, 253.

Bass, M. N. 1971, Geochimica et Cosmochimica Acta 35, 139 .

BeckIin, E. E. and Westphal, J. A. 1966, Ap. J. I45, 445 .

Bless, R. C. and Savage, B. D. 1972, Ap. J. 171, 293.

Cameron, A. G. W. 1973, in Interstellar Dust and Related Topics, I.A.U. Symposium No. 52, J. M. Greenberg and H. C. Van dé Hulst, Edicors (D. Reidel Publishing Co., Boston), p. 545.

Crampton, D., Cowley, A. P. and Humphreys, R. M. 1975, Ap. J. (Letters) 198, L135.

Cooke, S. R. B., 1976, Private Communication.

Day, K. L. 1974, Ap. J. (Letters) 192, LI5.

Day, K. L., Steyer, T. R. and Huffman, D. R. 1974 , Ap. J. 19I, 415 .

Day, K. L. 1975, Ap. J. 199, 660.

Day, K. L. 1976 , Ap. J. 210, 614.

Donn, B. D., Wickramasinghe, N. C., Hudson, J. P. and Stecker, T. P. 1968, Ap. J. 153, 451 .

Donn, B. 1976, Memoires Societe Royale des Sciences de Liege, $6^{\mathrm{e}}$ serie, tome IX, 499 , 
Dorschner, J., Gurtlen, J. and Friedemann, C., 1976, Astron. Nachr., Bd 297, H.3, 159.

Drummeter, L. F. and Hass, G. 1964, in Physics of Thin Films, Vol. 2, G. Hass and R. E. Thun, Ed. (Academic Press), pp. 336-338.

Duke, M. B. and Silver, L. T. 1967, Geochimica et Cosmochimica Acta 3I, 1637.

Forrest, W. J., Gillett, F. C. and Stein, W. A., 1975, Ap. J. 195, 423.

Gammon, R. H., Gaustad, J. E. and Treffers, R. R., I972, Ap. J. 175, 687 .

Gaustad, J. E. 1963, Ap. J. 138, 1050.

Gehrz, R. D., Ney, E. P. and Strecker, D. 1970, Ap. J. (Letters) 16I, L219.

Gehrz, R. D. and Woolf, N. J. 1971, Ap. J. I65, 285.

Gehrz, R. D., Ney, E. P., Becklin, E. E. and Neugebauer, G. 1973, Astrophysical Letters 13, 89 .

Gillett, F. C., Low, F. J. and Stein, W. A. 1968, Ap. J. 154, 677 .

Gillett, F. C. and Forrest, W. J. 1973, AP. J. I79, 483.

Gillett, F. C., Jones, T. W., Merrill, K. M. and Stein, W. A. 1975, Astronomy and Astrophysics 45, 77 .

Gillett, F. C. and Soifer, B. T. 1976, Ap. J. 207, 780 .

Gilman, R. C. 1969, Ap. J. (Letters) 155, LI85.

Gilra, D. P. 1971, Nature 229, 237.

Gold, T., Bilson, E. and Baron, R. L. 1976, The Search for the Cause of the Low Albedo of the Moon, Cornell University Center for Radiophysics and Space Research, Report No. CRSR 625, Ithaca, N.Y.). 
Grossman, L. and Larimer, J. W. 1974, Reviews of Geophysics and Space Physics 12, 71.

Hass, G. and Salzberg, C. D. 1954, Journal of the Optical Society of America 44, 181.

Heiken, G. 1974, A Catalogue of Lunar Soils (NASA, Lyndon B. Johnson Space Center).

Herbig, G. H. 1972, Ap. J. 172, 375.

Huffman, D. R. and Stapp, J. I. 197I, Nature Physical Science 229,45 .

Humphreys, R. M., Strecker, D. W. and Ney, E. P. 1971, Ap. J. (Letters) I67, L35.

Humphreys, R. M., Strecker, D. W. and Ney, E. P. 1972, Ap. J. I72, 75 .

Hunt, G. R. and Logan, L. M. 1972, ADplied Optics II, 142 .

Hunt, G. R., Logan, L. M. and Salisbury, J. W., 1973, Icarus 18,459 .

Hunt, G. R. and Salisbury, J. W. 1974, Mid-Infrared Spectral Behavior of Igneous Rocks (Air Force Cambridge Research Laboratories, Report Number AFCRL-TR-74-0625.)

Hunt, J. M., Wisherd, M. P. and Bonham, L. C. 1950, Analytical Chemistry 22, 1478 .

Hyland, A. R. and Neugebauen, G. 1970, Ap. J. (Letters) 160, LI77.

Johnson, H. L. 1966, Sky and Telescope 32, 73 .

Jones, T. W. and Merrill, K. M. 1976, Ap. J. 209, 509.

Launer, P. J. 1952, Am. Miner 37, 76!.

Logan, L. M. and Hunt, G. R. 1970, Journal of Geophysical Research 75, 6539 .

Logan, L. M., Hunt, G. R., Balsamo, S, R. and Salisbury, J.W. 1972, Procieedings of the Thind Lunar Science Conference (Supplement 3, Geochimica et Cosmochimica Acta) 3, 3069. 
Low, F. J. and Swamy, K. 1970, Nature 227, 1333.

Lyon, R. J. P. 1964, Evaluation of Infrared Spectrophotometry for Compositional Analys is of Lunar and Planetary Solls, Part II: Rough and Powdered Surfaces, NASA Contractor Report CR-100.

Maas, R. W., Ney, E. P. and Woolf, N. J. 1970, Ap. J. (Letters) 160, IIOI.

Merrill, K. M. 1974, Icarus 23, 566 .

Merrill, K. M. and Soifer, B. T. 1974, Ap. J. (Letters) I89, L27.

Merrill, K. M. and Stein, W. A. 1976a, P.A.S.P. 88, 285 .

Merrill, K. M. and Stein, W. A. 1976b, P.A.S.P. $\underline{88}$, 294.

Merrill, K. M., RusseIl, R. W. and Soifer, B. T. $19.76 \mathrm{c}$, Ap. J. 207, 763 .

Meyer, C. Jr. 1971, Geochimica et Cosmochimica Acta 35, 551 .

Ney, E. P., Strecker, D. W. and Gehrz, R. D. 1973, Ap. J. 180,809 .

Ney, E. P. I974a, Ap. J. (Letters) 189, LI4I.

Ney, E. P. 1974b, Icarus 23, 551.

Ney, E. P. 1975, Sky and Telescope 49, 21.

Ney, R. P. and Merrill, K. M. 1976, Science 194, 1051.

Ney, E. P. 1977a, Science 195, 541.

Ney, R. P. 1977b, Seminar Presentation.

Nyquist, R. A. and Kagel, R. 0. 1971, Infrared

Spectra of Inorganic Compounds (Academic Press).

O'Keefe, J. A. 1939, Ap. J. 90, 294.

Oort, J. H. 1932, B.A. N., NO. 238. 
Orr, C. and Dallavalle, J. M. 1959, Fine Paricicle Measurement (Macmillan) p. 44.

Pfund, A. H. 1933, Journal of the Optical Society of America 23, 375 .

Pfund, A. H. 1934, Journal of the Optical Society of America 2느, 143 .

Russe11, R. W., Soifer, B. T. and Forrest, W. J. 1975, Ap. J. (Letters). 198, L4l.

Sekanina, Z. 1974, Sky and Telescope 47, 374.

Simon, I. and McMahon, H. O. 1953, Journal of Chemical Physics 2I, 23.

Stebbins, J., Huffer, C. M. and Whitford, A. E. 1939, Ap. J. 90, 209 .

Stecher, T. P. and Donn B. 1965, Ap. J. 142, 1681.

Stein, W. A., Gaustad, J. E., Gillett, F. C. and Knacke, R. F. 1969a, Ap. J. (Letters) 155, L3.

Stein, W. A. and Gillett, F. 1969D, Ap. J. (Letters) I55, LI97.

Steyer, T. R. 1974, Ph. D. Thesis, University of Arizona.

Steyer, T. R., Day, K. L. and Huffman, D. R, 1974, Applied Optics, I3, 1586.

Strecker, D. W. and Ney, E. P. 1974, Astronomical Jounnal $\underline{79}, 797$.

Struye, F. G. W. 1847, Etudes d'Astronomie Stellaire.

Treffers, R. and Cohen, M. 1974, Ap. J. 188, 545.

Trumpler, R. J. 1930, P.A.S.P. 42, 214.

Van de Hulst, H. C. 1957, Light Scattering by Small Particles (Wiley).

Wickramasinghe, N. C. i967, Interstellar Grains, (Chapman and Hall, London). 
Wood, J. A. 1963, in The Moon, Meteorites and Comets, B. M. Middlehurst and G. P. Kuiper, Ed. (University ó Chicago Press, Chicago), p. 337.

Wood, J. A. 1968, Meteorites and the Origin of Planets (McGraw-Hill), PP. 77-84.

Wood, J. A. 1975, Scientific American 233, September, 92 .

Woolf, N. J. 1973a, in Interstellar Dust and Related Topics, I.A.U. Symposium No. 52, J. M. Greenburg and H. C. Van de Hulst, Eds (D. Reidel Publ. Co., Boston), p. 485 .

Woolf, N. J. 19735, in The Dusty Universe, G. B. Field and A.G.W. Cameron, Eds. (Neale Watson Academic Publications, Inc., N. Y.) p. 59.

Woolf, N. J. and Ney, E. P. I969, Ap. J. (Letters) 155, LI81.

Zaikowski, A., Knacke, R. F. and Porco, C. C. 1975a, Astrophysics and space Science $\underline{35}, 97$.

Zaikowski, A. and Knacke, R. F. 1975b, Astrophysics and Space Science 37,3 . 